Article

\title{
Stability Results for a Coupled System of Impulsive Fractional Differential Equations
}

\author{
Akbar Zada ${ }^{1} \oplus$, Shaheen Fatima ${ }^{1}$, Zeeshan Ali ${ }^{1}{ }^{\circledR}$, Jiafa $\mathrm{Xu}^{2}$ and Yujun Cui ${ }^{3, *}$ \\ 1 Department of Mathematics, University of Peshawar, Khyber Pakhtunkhwa 25000, Pakistan; \\ akbarzada@uop.edu.pk (A.Z.); shaheenfatima072@gmail.com (S.F.); zeeshanmaths1@gmail.com (Z.A.) \\ 2 School of Mathematical Sciences, Chongqing Normal University, Chongqing 401331, China; \\ 20150028@cqnu.edu.cn \\ 3 State Key Laboratory of Mining Disaster Prevention and Control Co-founded by Shandong Province and the \\ Ministry of Science and Technology, Shandong University of Science and Technology, Qingdao 266590, China \\ * Correspondence: cyj720201@sdust.edu.cn
}

Received: 2 September 2019; Accepted: 2 October 2019; Published: 6 October 2019

check for updates

\begin{abstract}
In this paper, we establish sufficient conditions for the existence, uniqueness and UlamHyers stability of the solutions of a coupled system of nonlinear fractional impulsive differential equations. The existence and uniqueness results are carried out via Banach contraction principle and Schauder's fixed point theorem. The main theoretical results are well illustrated with the help of an example.
\end{abstract}

Keywords: caputo fractional derivative; coupled system; impulses; existence theory; stability theory

\section{Introduction}

Fractional differential equations (FDEs) provide an excellent tool for the description of memory and hereditary properties of different processes and materials. Thus, contrary to the classical derivative, the fractional derivative is nonlocal. Fractional calculus has played a very important role in enhancing the mathematical modeling of several phenomena occurring in engineering and scientific disciplines, such as blood flow systems, control theory, aerodynamics, the nonlinear oscillation of earthquake, the fluid-dynamic traffic model, polymer rheology, regular variation in thermodynamics, etc. FDEs are more accurate than the integer-order derivatives. Therefore, in the last few decades, fractional calculus has received great attention from researchers [1-13]. On the other hand, it is impossible to describe the complicated systems and processes with a single differential equation. Therefore, the coupled systems involving FDEs have also received incredible attention; consequently, many results are devoted to them [14-31].

It is well known that the effects of a pulse cannot be ignored in many processes and phenomena. For example, in biological systems such as heart beats, blood flows, mechanical systems with impact, population dynamical systems and so on. Thus, researchers used differential equations with impulses to describe the aforesaid kinds of phenomena. Therefore, many mathematicians studied impulsive FDEs with different boundary conditions; see [32-40] and references cited therein.

In fields such as numerical analysis, optimization theory, and nonlinear analysis, we mostly deal with the approximate solutions and hence we need to check how close these solutions are to the actual solutions of the related system. For this purpose, many approaches can be used, but the approach of Ulam-Hyers stability is a simple and easy one. The aforesaid stability was first initiated by Ulam in 1940 and then was confirmed by Hyers in 1941 [41,42]. That's why this stability is known as Ulam-Hyers stability. In 1978 [43], Rassias generalized the Ulam-Hyers stability by considering 
variables. Thereafter, mathematicians extended the work mentioned above to functional, differential, integrals and FDEs; for more information about the topic, the reader is recommended to [44-59].

Inspired from the above discussion, in this article, we study the existence, uniqueness and stability analysis of a coupled system of nonlinear FDEs with impulses of the form:

$$
\left\{\begin{array}{l}
{ }^{c} D^{\alpha} x(t)+h\left(t^{c}{ }^{c} D^{a} x(t),{ }^{c} D^{b} y(t)\right)=0, t \neq t_{m}, m=1,2 \ldots, n, \\
{ }^{c} D^{\beta} y(t)+w\left(t,{ }^{c} D^{a} x(t),{ }^{c} D^{b} y(t)\right)=0, t \neq t_{m}, m=1,2 \ldots, n, \\
\left.\Delta x\right|_{t=t_{m}}=M_{1 m}\left(x\left(t_{m}\right)\right),\left.\Delta x^{\prime}\right|_{t=t_{m}}=N_{1 m}\left(x\left(t_{m}\right)\right),\left.\Delta x^{\prime \prime}\right|_{t=t_{m}}=O_{1 m}\left(x\left(t_{m}\right)\right) \\
\left.\Delta y\right|_{t=t_{m}}=M_{2 m}\left(y\left(t_{m}\right)\right),\left.\Delta y^{\prime}\right|_{t=t_{m}}=N_{2 m}\left(y\left(t_{m}\right)\right),\left.\Delta y^{\prime \prime}\right|_{t=t_{m}}=O_{2 m}\left(y\left(t_{m}\right)\right) \\
x(0)=x^{\prime}(0)=0,{ }^{c} D^{\varepsilon} x(\Omega)=x^{\prime \prime}(1) \\
y(0)=y^{\prime}(0)=0,{ }^{c} D^{\rho} y(\Phi)=y^{\prime \prime}(1)
\end{array}\right.
$$

where $\mathrm{t} \in \mathrm{J}=[0,1], 2<\alpha, \beta \leqslant 3,0<\mathrm{a}, \mathrm{b}, \varepsilon, \Omega, \rho, \Phi<1 .{ }^{\mathrm{c}} \mathbf{D}$ stands for Caputo fractional derivative and $h, w: J \times \mathbb{R}^{3} \rightarrow \mathbb{R}$ are continuous functions. $M_{1 m}, M_{2 m}, N_{1 m}, N_{2 m}, O_{1 m}, O_{2 m} \in$ $\mathrm{C}(\mathbb{R}, \mathbb{R})$ and $\mathrm{t}_{\mathrm{m}}$ satisfied $0=\mathrm{t}_{0}<\mathrm{t}_{1}<\cdots<\mathrm{t}_{\mathrm{n}}<\mathrm{t}_{\mathrm{n}+1}=1,\left.\Delta x\right|_{\mathrm{t}=\mathrm{t}_{\mathrm{m}}}=\mathrm{x}\left(\mathrm{t}_{\mathrm{m}}^{+}\right)-\mathrm{x}\left(\mathrm{t}_{\mathrm{m}}^{-}\right),\left.\Delta \mathrm{x}^{\prime}\right|_{\mathrm{t}=\mathrm{t}_{\mathrm{m}}}=$ $x^{\prime}\left(t_{m}^{+}\right)-x^{\prime}\left(t_{m}^{-}\right),\left.\Delta x^{\prime \prime}\right|_{t=t_{m}}=x^{\prime \prime}\left(t_{m}^{+}\right)-x^{\prime \prime}\left(t_{m}^{-}\right),\left.\Delta y\right|_{t=t_{m}}=y\left(t_{m}^{+}\right)-y\left(t_{m}^{-}\right),\left.\Delta y^{\prime}\right|_{t=t_{m}}=y^{\prime}\left(t_{m}^{+}\right)-$ $y^{\prime}\left(t_{m}^{-}\right),\left.\Delta y^{\prime \prime}\right|_{t=t_{m}}=y^{\prime \prime}\left(t_{m}^{+}\right)-y^{\prime \prime}\left(t_{m}^{-}\right), x\left(t_{m}^{+}\right), y\left(t_{m}^{+}\right)$, and $x\left(t_{m}^{-}\right), y\left(t_{m}^{-}\right)$represent the right and left limits of $x(t), y(t)$, respectively, at $t=t_{m}$.

The remaining article is organized as follows: In Section 2, we give some definitions and lemmas related to fractional calculus. In Section 3, we establish our main results about the existence and uniqueness of solutions for the proposed system (1). In Section 4, we study the Ulam-Hyers stability. In Section 5, we provide an example to support our main results.

\section{Background Materials}

In this section, we give some basic definitions of fractional calculus that will be used throughout the article.

Definition 1. (see [60]) If $x:(0, \infty) \rightarrow \mathbb{R}$ and $\alpha>0$, then the Caputo fractional derivative of order $\alpha$ is defined as

$$
{ }^{c} D^{\alpha} \chi(t)=\frac{1}{\Gamma(n-\alpha)} \int_{0}^{t}(t-s)^{n-\alpha-1} \chi^{(n)}(s) d s, n-1<\alpha<n, n=[\alpha]+1,
$$

where $[\alpha]$ denotes the integer part of real number $\alpha$, provided that the right side is pointwise defined on $(0, \infty)$.

Definition 2. (see [60]) The Riemann-Liouville fractional integral of order $\alpha>0$ for a function $x:(0, \infty) \rightarrow \mathbb{R}$ is defined as

$$
\mathbf{I}^{\alpha} x(t)=\frac{1}{\Gamma(\alpha)} \int_{0}^{t}(t-s)^{\alpha-1} x(s) d s, \quad t>0,
$$

provided that the right side is pointwise defined on $(0, \infty)$, where $\Gamma$ is the Euler Gamma function.

Lemma 1. (see [60]) The solution of the differential equations involving Caputo derivative ${ }^{\mathrm{c}} \mathrm{D}^{\alpha} \mathrm{x}(\mathrm{t})=\mathrm{f}(\mathrm{t})$, $\mathrm{t} \in \mathrm{J}$, has the form:

$$
\mathbf{I}^{\alpha c} \mathbf{D}^{\alpha} \chi(\mathbf{t})=\mathbf{I}^{\alpha} \mathbf{f}(\mathrm{t})+e_{0}+e_{1} t+\cdots+e_{n-1} t^{n-1},
$$

for some $e_{i} \in \mathbb{R}, i=0,1, \ldots, n-1, n=[\alpha]+1$.

Lemma 2. (see [60]) If $\alpha, \beta>0, \mathrm{t} \in \mathrm{J}$, then, for $\mathrm{x}(\mathrm{t})$, we have

$$
{ }^{c} D^{\alpha} I^{\alpha} x(t)=x(t), \quad I^{\alpha} I^{\beta} x(t)=I^{\alpha+\beta} x(t) .
$$


Lemma 3. (Banach contraction principle, see [59]) If $X$ is real Banach space and $\mathbf{W}: X \longrightarrow X$ is a contraction mapping, then $\mathbf{W}$ has a unique fixed point in $\mathrm{X}$.

Theorem 1. (Schauder fixed point theorem, see [59]) If $\omega$ is a closed bounded convex subset of a Banach space $\mathrm{X}$ and $\mathbf{W}: \omega \longrightarrow \omega$ is completely continuous, then $\mathbf{W}$ has at least one fixed point in $\omega$.

For the sake of convenience, we introduce the Banach space as follows:

Let $\mathrm{J}=[0,1], \mathrm{J}^{\prime}=\mathrm{J} /\left\{\mathrm{t}_{1}, \mathrm{t}_{2}, \ldots, \mathrm{t}_{\mathrm{n}}\right\}$. Define the set by

$$
\begin{aligned}
X=P C(J)= & \left\{x(t): x(t), x^{\prime}(t), x^{\prime \prime}(t),{ }^{c} D^{a} x(t),{ }^{c} D^{b} x(t) \in C\left(J^{\prime}\right), x\left(t_{m}^{+}\right) \text {and } x\left(t_{m}^{-}\right)\right. \\
& \text {exists and satisfying } \left.x\left(t_{m}^{-}\right)=x\left(t_{m}\right), 1 \leqslant m \leqslant n\right\} .
\end{aligned}
$$

It is easy to verify that $X$ is a Banach space equipped with the norm:

$$
\|x\|_{0}=\max \left\{\sup _{t \in J}|x(t)|, \sup _{t \in J}\left|x^{\prime}(t)\right|, \sup _{t \in J}\left|x^{\prime \prime}(t)\right|, \sup _{t \in J}\left|{ }^{c} D^{a} x(t)\right|, \sup _{t \in J}\left|{ }^{c} D^{b} x(t)\right|\right\}, \forall x(t) \in P C(J) .
$$

Similarly, we can define a set $\mathrm{Y}=\mathrm{PC}(\mathrm{J})$, which is a Banach space endowed with the defined norm:

$$
\|y\|_{0}=\max \left\{\sup _{t \in J}|y(t)|, \sup _{t \in J}\left|y^{\prime}(t)\right|, \sup _{t \in J}\left|y^{\prime \prime}(t)\right|, \sup _{t \in J}\left|{ }^{c} D^{a} y(t)\right|,\left.\sup _{t \in J}\right|^{c} D^{b} y(t) \mid\right\}, \forall y(t) \in P C(J) .
$$

Furthermore, we define the Banach space $Y^{\prime}=X \times Y$ with the norms $\|(x, y)\|=\|x\|_{0}+\|y\|_{0}$ and $\|(x, y)\|=\max \left\{\|x\|_{0},\|y\|_{0}\right\}$.

Definition 3. A pair of functions $(x(t), y(t)) \in Y^{\prime}$ is called a solution of $(1)$ if $(x(t), y(t))$ satisfy all the equations and boundary value conditions of the system (1).

Lemma 4. Assume that $\mathrm{f} \in \mathrm{C}(\mathrm{J}, \mathrm{IR})$. A function $\mathrm{x} \in \mathrm{PC}(\mathrm{J})$ is a solution of the boundary value system

$$
\left\{\begin{array}{l}
{ }^{c} D^{\alpha} x(t)+f(t)=0,2<\alpha \leqslant 3 \\
\left.\Delta x\right|_{t=t m}=M_{1 m}\left(x\left(t_{m}\right)\right), m=1,2, \ldots, n \\
\left.\Delta x^{\prime}\right|_{t=t m}=N_{1 m}\left(x\left(t_{m}\right)\right), m=1,2, \ldots, n, \\
\left.\Delta x^{\prime \prime}\right|_{t=t m}=O_{1 m}\left(x\left(t_{m}\right)\right), m=1,2, \ldots, n, \\
x(0)=x^{\prime}(0)=0,{ }^{c} D^{\varepsilon} x(\Omega)=x^{\prime \prime}(1), 0<\varepsilon, \Omega<1
\end{array}\right.
$$

if and only if $\mathrm{x} \in \mathrm{PC}(\mathrm{J})$ is the solution of integral equation

$$
x(t)=\left\{\begin{array}{l}
-\frac{1}{\Gamma(\alpha)} \int_{0}^{t}(t-s) f(s) d s+e^{*} t^{2}, t \in\left[0, t_{1}\right], \\
-\frac{1}{\Gamma(\alpha)} \int_{t_{m}}^{t}(t-s)^{\alpha-1} f(s) d s-\frac{1}{\Gamma(\alpha)} \sum_{j=1} \int_{t_{j-1}}^{t_{j}}\left(t_{j}-s\right)^{\alpha-1} f(s) d s \\
-\frac{1}{\Gamma(\alpha-1)} \sum_{j=1}\left(t-t_{j}\right) \int_{t_{j-1}}^{t_{j}}\left(t_{j}-s\right)^{\alpha-2} f(s) d s-\frac{1}{2 \Gamma(\alpha-2)} \sum_{j=1}^{m}\left(t-t_{j}\right)^{2} \\
\times \int_{t_{j-1}}^{t_{j}}\left(t_{j}-s\right)^{\alpha-3} f(s) d s+\sum_{j=1} M_{1 j}\left(x\left(t_{j}\right)\right)+\sum_{j=1}\left(t-t_{j}\right) N_{1 j}\left(x\left(t_{j}\right)\right) \\
+\sum_{j=1}^{m} \frac{\left(t-t_{j}\right)^{2}}{2} O_{1 j}\left(x\left(t_{j}\right)\right)+e^{*} t^{2}, t \in\left(t_{m}, t_{m+1}\right], 1 \leqslant m \leqslant n,
\end{array}\right.
$$

where $t \in\left(t_{m}, t_{m+1}\right], 1 \leqslant m \leqslant n$, 


$$
\begin{aligned}
e^{*}= & \frac{\Gamma(3-\varepsilon)}{2\left(\Gamma(3-\varepsilon)-\Omega^{2-\varepsilon}\right)}\left[-\frac{1}{\Gamma(\alpha-\varepsilon)} \int_{t_{j}}^{\Omega}(\Omega-s)^{\alpha-\varepsilon-1} f(s) d s-\frac{\Omega^{1-\varepsilon}}{\Gamma(\alpha-1) \Gamma(2-\varepsilon)}\right. \\
& \times \sum_{j=1}^{m} \int_{t_{j-1}}^{t_{j}}\left(t_{j}-s\right)^{\alpha-2} f(s) d s-\frac{\Omega^{2-\varepsilon}}{\Gamma(\alpha-2) \Gamma(3-\varepsilon)} \sum_{j=1}^{m} \int_{t_{j-1}}^{t_{j}}\left(t_{j}-s\right)^{\alpha-3} f(s) d s \\
& +\frac{\Omega^{1-\varepsilon}}{\Gamma(\alpha-2) \Gamma(2-\varepsilon)} \sum_{j=1}^{m} t_{j} \int_{t_{j-1}}^{t_{j}}\left(t_{j}-s\right)^{\alpha-3} f(s) d s+\frac{\Omega^{1-\varepsilon}}{\Gamma(2-\varepsilon)} \sum_{j=1}^{m} N_{1 j}\left(x\left(t_{j}\right)\right) \\
& +\frac{\Omega^{2-\varepsilon}}{\Gamma(3-\varepsilon)} \sum_{j=1}^{m} O_{1 j}\left(x\left(t_{j}\right)\right)-\frac{\Omega^{1-\varepsilon}}{\Gamma(2-\varepsilon)} \sum_{j=1}^{m} t_{j} O_{1 j}\left(x\left(t_{j}\right)\right)+\frac{1}{\Gamma(\alpha-2)} \int_{t_{m}}^{1}(1-s)^{\alpha-3} f(s) d s \\
& \left.+\frac{1}{\Gamma(\alpha-2)} \sum_{j=1}^{m} \int_{t_{j-1}}^{t_{j}}\left(t_{j}-s\right)^{\alpha-3} f(s) d s-\sum_{j=1}^{m} O_{1 j}\left(x\left(t_{j}\right)\right)\right] .
\end{aligned}
$$

Proof. Applying Lemma 1, for some constants $e_{0}, e_{1}, e_{2} \in \mathbb{R}$, we have

$$
\begin{aligned}
x(t) & =-I^{\alpha} f(s) d s+e_{0}+e_{1} t+e_{2} t^{2} \\
& =-\frac{1}{\Gamma(\alpha)} \int_{0}^{t}(t-s)^{\alpha-1} f(s) d s+e_{0}+e_{1} t+e_{2} t^{2}, t \in\left[0, t_{1}\right] .
\end{aligned}
$$

Then, we obtain

$$
\left\{\begin{array}{l}
x^{\prime}(t)=-\frac{1}{\Gamma(\alpha-1)} \int_{0}^{t}(t-s)^{\alpha-2} f(s) d s+e_{1}+2 e_{2} t \\
x^{\prime \prime}(t)=-\frac{1}{\Gamma(\alpha-2)} \int_{0}^{t}(t-s)^{\alpha-3} f(s) d s+2 e_{2} .
\end{array}\right.
$$

When $t \in\left(t_{1}, t_{2}\right)$, we have

$$
\left\{\begin{array}{l}
x(t)=-\frac{1}{\Gamma(\alpha)} \int_{t_{1}}^{t}(t-s)^{\alpha-1} f(s) d s+e_{3}+e_{4}\left(t-t_{1}\right)+e_{5}\left(t-t_{1}\right)^{2} \\
x^{\prime}(t)=-\frac{1}{\Gamma(\alpha-1)} \int_{t_{1}}^{t}(t-s)^{\alpha-2} f(s) d s+e_{4}+2 e_{5}\left(t-t_{1}\right) \\
x^{\prime \prime}(t)=-\frac{1}{\Gamma(\alpha-2)} \int_{t_{1}}^{t}(t-s)^{\alpha-3} f(s) d s+2 e_{5}
\end{array}\right.
$$

where $e_{3}, e_{4}, e_{5}$ are arbitrary constants, from (4)-(6), we can find

$$
\begin{gathered}
x\left(t_{1}^{-}\right)=-\frac{1}{\Gamma(\alpha)} \int_{0}^{t_{1}}\left(t_{1}-s\right)^{\alpha-1} f(s) d s+e_{0}+e_{1} t_{1}+e_{2} t_{1}^{2}, \quad x\left(t_{1}^{+}\right)=e_{3}, \\
x^{\prime}\left(t_{1}^{-}\right)=-\frac{1}{\Gamma(\alpha-1)} \int_{0}^{t_{1}}\left(t_{1}-s\right)^{\alpha-2} f(s) d s+e_{1}+2 e_{2} t_{1}, \quad x^{\prime}\left(t_{1}^{+}\right)=e_{4}, \\
x^{\prime \prime}\left(t_{1}^{-}\right)=-\frac{1}{\Gamma(\alpha-2)} \int_{0}^{t_{1}}\left(t_{1}-s\right)^{\alpha-3} f(s) d s+2 e_{2}, \quad x^{\prime \prime}\left(t_{1}^{+}\right)=2 e_{5} .
\end{gathered}
$$

Furthermore, $\left.\Delta x\right|_{t=t_{1}}=M_{11}\left(x\left(t_{1}\right)\right),\left.\Delta x^{\prime}\right|_{t=t_{1}}=N_{11}\left(x\left(t_{1}\right)\right),\left.\Delta x^{\prime \prime}\right|_{t=t_{1}}=O_{11}\left(x\left(t_{1}\right)\right)$, and (7)-(9) give us:

$$
\left\{\begin{array}{l}
e_{3}=-\frac{1}{\Gamma(\alpha)} \int_{0}^{t_{1}}\left(t_{1}-s\right)^{\alpha-1} f(s) d s+e_{0}+e_{1} t_{1}+e_{2} t_{1}^{2}+M_{11}\left(x\left(t_{1}\right)\right), \\
e_{4}=-\frac{1}{\Gamma(\alpha-1)} \int_{0}^{t_{1}}\left(t_{1}-s\right)^{\alpha-2} f(s) d s+e_{1}+2 e_{2} t_{1}+N_{11}\left(x\left(t_{1}\right)\right) \\
e_{5}=-\frac{1}{2 \Gamma(\alpha-2)} \int_{0}^{t_{1}}\left(t_{1}-s\right)^{\alpha-3} f(s) d s+e_{2}+\frac{1}{2} O_{11}\left(x\left(t_{1}\right)\right) .
\end{array}\right.
$$


Plugging $e_{3}, e_{4}$, and $e_{5}$ into the first equation of (6) for $t \in\left(t_{1}, t_{2}\right]$, we have

$$
\begin{aligned}
x(t)= & -\frac{1}{\Gamma(\alpha)} \int_{t_{1}}^{t}(t-s)^{\alpha-1} f(s) d s-\frac{1}{\Gamma(\alpha)} \int_{0}^{t_{1}}\left(t_{1}-s\right) f(s) d s-\frac{\left(t-t_{1}\right)}{\Gamma(\alpha-1)} \int_{0}^{t_{1}}\left(t_{1}-s\right)^{\alpha-2} f(s) d s \\
& -\frac{\left(t-t_{1}\right)^{2}}{2 \Gamma(\alpha-2)} \int_{0}^{t_{1}}\left(t_{1}-s\right)^{\alpha-3} f(s) d s+M_{11}\left(x\left(t_{1}\right)\right)+\left(t-t_{1}\right) N_{11}\left(x\left(t_{1}\right)\right)+\frac{\left(t-t_{1}\right)^{2}}{2} O_{11}\left(x\left(t_{1}\right)\right) \\
& +e_{0}+e_{1} t+e_{2} t^{2} .
\end{aligned}
$$

Repeating the same process for $t \in\left(t_{m}, t_{m+1}\right]$ such that $(m=1,2, \ldots, n)$, then we can write

$$
\begin{aligned}
x(t)= & -\frac{1}{\Gamma(\alpha)} \int_{t_{m}}^{t}(t-s)^{\alpha-1} f(s) d s-\frac{1}{\Gamma(\alpha)} \sum_{j=1}^{m} \int_{t_{j-1}}^{t_{j}}\left(t_{j}-s\right)^{\alpha-1} f(s) d s-\frac{1}{\Gamma(\alpha-1)} \sum_{j=1}^{m}\left(t-t_{j}\right) \\
& \times \int_{t_{j-1}}^{t_{j}}\left(t_{j}-s\right)^{\alpha-2} f(s) d s-\frac{1}{2 \Gamma(\alpha-2)} \sum_{j=1}^{m}\left(t-t_{j}\right)^{2} \int_{t_{j-1}}^{t_{j}}\left(t_{j}-s\right)^{\alpha-3} f(s) d s+\sum_{j=1}^{m} M_{1 j}\left(x\left(t_{j}\right)\right) \\
& +\sum_{j=1}^{m}\left(t-t_{j}\right) N_{1 j}\left(x\left(t_{j}\right)\right)+\sum_{j=1}^{m} \frac{\left(t-t_{j}\right)^{2}}{2} O_{1 j}\left(x\left(t_{j}\right)\right)+e_{0}+e_{1} t+e_{2} t^{2} .
\end{aligned}
$$

Furthermore, we have

$$
\begin{aligned}
x^{\prime \prime}(t)= & -\frac{1}{\Gamma(\alpha-2)} \int_{t_{m}}^{t}(t-s)^{\alpha-3} f(s) d s-\frac{1}{\Gamma(\alpha-2)} \sum_{j=1}^{m} \int_{t_{j-1}}^{t_{j}}\left(t_{j}-s\right)^{\alpha-3} f(s) d s+O_{1 j}\left(x\left(t_{j}\right)\right) \\
& +2 e_{2}
\end{aligned}
$$

By utilizing conditions $x(0)=x^{\prime}(0)=0$ in (4), we get $e_{0}=e_{1}=0$. In addition, it follows from (11) that

$$
\begin{aligned}
x^{\prime \prime}(1)= & -\frac{1}{\Gamma(\alpha-2)} \int_{t_{m}}^{1}(1-s)^{\alpha-3} f(s) d s-\frac{1}{\Gamma(\alpha-2)} \sum_{j=1}^{m} \int_{t_{j-1}}^{t_{j}}\left(t_{j}-s\right)^{\alpha-3} f(s) d s+O_{1 j}\left(x\left(t_{j}\right)\right) \\
& +2 e_{2} .
\end{aligned}
$$

In view of $j \in\{0,1, \ldots, n\}$ such that $\Omega \in\left(t_{j}, t_{j+1}\right]$, we have

$$
\begin{aligned}
x(\Omega)= & -\frac{1}{\Gamma(\alpha)} \int_{t_{j}}^{\Omega}(\Omega-s)^{\alpha-1} f(s) d s-\frac{1}{\Gamma(\alpha)} \sum_{j=1}^{m} \int_{t_{j-1}}^{t_{j}}\left(t_{j}-s\right)^{\alpha-1} f(s) d s-\frac{1}{\Gamma(\alpha-1)} \sum_{j=1}^{m}\left(\Omega-t_{j}\right) \\
& \times \int_{t_{j-1}}^{t_{j}}\left(t_{j}-s\right)^{\alpha-2} f(s) d s-\frac{1}{2 \Gamma(\alpha-2)} \sum_{j=1}^{m}\left(\Omega-t_{j}\right)^{2} \int_{t_{j-1}}^{t_{j}}\left(t_{j}-s\right)^{\alpha-3} f(s) d s \\
& +\sum_{j=1}^{m} M_{1 j}\left(x\left(t_{j}\right)\right)+\sum_{j=1}^{m}\left(\Omega-t_{j}\right) N_{1 j}\left(x\left(t_{j}\right)\right)+\frac{\left(\Omega-t_{j}\right)^{2}}{2} O_{1 j}\left(x\left(t_{j}\right)\right)+e_{2} \Omega^{2} .
\end{aligned}
$$

By applying result (5), we get

$$
\begin{aligned}
{ }^{c} D_{0^{+}}^{\varepsilon} x(\Omega)= & -\frac{1}{\Gamma(\alpha-\varepsilon)} \int_{t_{j}}^{\Omega}(\Omega-s)^{\alpha-\varepsilon-1} f(s) d s-\frac{\Omega^{1-\varepsilon}}{\Gamma(\alpha-1) \Gamma(2-\varepsilon)} \sum_{j=1}^{m} \int_{t_{j-1}}^{t_{j}}\left(t_{j}-s\right)^{\alpha-2} f(s) d s \\
& -\frac{\Omega^{2-\varepsilon}}{\Gamma(\alpha-2) \Gamma(3-\varepsilon)} \sum_{j=1}^{m} \int_{t_{j-1}}^{t_{j}}\left(t_{j}-s\right)^{\alpha-3} f(s) d s+\frac{\Omega^{1-\varepsilon}}{\Gamma(\alpha-2) \Gamma(2-\varepsilon)} \sum_{j=1}^{m} t_{j} \\
& \times \int_{t_{j-1}}^{t_{j}}\left(t_{j}-s\right)^{\alpha-3} f(s) d s+\frac{\Omega^{1-\varepsilon}}{\Gamma(2-\varepsilon)} \sum_{j=1}^{m} N_{1 j}\left(x\left(t_{j}\right)\right)+\frac{\Omega^{2-\varepsilon}}{\Gamma(3-\varepsilon)} \sum_{j=1}^{m} O_{1 j} \\
& -\frac{\Omega^{1-\varepsilon}}{\Gamma(2-\varepsilon)} \sum_{j=1}^{m} t_{j} O_{1 j}+\frac{2 \Omega^{2-\varepsilon}}{\Gamma(3-\varepsilon)} e_{2} .
\end{aligned}
$$


Since ${ }^{c} D_{0^{+}}^{\varepsilon} \chi(\Omega)=\chi^{\prime \prime}(1)$, thus (13) and (14) gives

$$
\begin{aligned}
e_{2}= & \frac{\Gamma(3-\varepsilon)}{2\left(\Gamma(3-\varepsilon)-\Omega^{2-\varepsilon}\right)}\left[-\frac{1}{\Gamma(\alpha-\varepsilon)} \int_{t_{j}}^{\Omega}(\Omega-s)^{\alpha-\varepsilon-1} f(s) d s-\frac{\Omega^{1-\varepsilon}}{\Gamma(\alpha-1) \Gamma(2-\varepsilon)}\right. \\
& \times \sum_{j=1}^{m} \int_{t_{j-1}}^{t_{j}}\left(t_{j}-s\right)^{\alpha-2} f(s) d s-\frac{\Omega^{2-\varepsilon}}{\Gamma(\alpha-2) \Gamma(3-\varepsilon)} \sum_{j=1}^{m} \int_{t_{j-1}}^{t_{j}}\left(t_{j}-s\right)^{\alpha-3} f(s) d s \\
& +\frac{\Omega^{1-\varepsilon}}{\Gamma(\alpha-2) \Gamma(2-\varepsilon)} \sum_{j=1}^{m} t_{j} \int_{t_{j-1}}^{t_{j}}\left(t_{j}-s\right)^{\alpha-3} f(s) d s+\frac{\Omega^{1-\varepsilon}}{\Gamma(2-\varepsilon)} \sum_{j=1}^{m} N_{1 j}\left(x\left(t_{j}\right)\right) \\
& +\frac{\Omega^{2-\varepsilon}}{\Gamma(3-\varepsilon)} \sum_{j=1}^{m} O_{1 j}-\frac{\Omega^{1-\varepsilon}}{\Gamma(2-\varepsilon)} \sum_{j=1}^{m} t_{j} O_{1 j}+\frac{1}{\Gamma(\alpha-2)} \int_{t_{m}}^{1}(1-s)^{\alpha-3} f(s) d s \\
& \left.+\frac{1}{\Gamma(\alpha-2)} \sum_{j=1}^{m} \int_{t_{j-1}}^{t_{j}}\left(t_{j}-s\right)^{\alpha-3} f(s) d s-\sum_{j=1}^{m} O_{1 j}\left(x\left(t_{j}\right)\right)\right] .
\end{aligned}
$$

Plugging the values of $e_{0}, e_{1}$ and $e_{2}$ into (4) and (10), (3) can thus be obtained. Conversely, we consider that $x(t)$ is a solution of (3). Then, it is obvious that (3) satisfies (2).

Similarly as in Lemma 4, we can prove the following:

Lemma 5. Let $\vartheta \in \mathrm{C}(\mathrm{J}, \mathbb{R})$. A function $\mathrm{y} \in \mathrm{PC}(\mathrm{J})$ is the solution of

$$
\left\{\begin{array}{l}
{ }^{c} D^{\beta} y(t)+\vartheta(t)=0,2<\beta \leqslant 3, \\
\left.\Delta y\right|_{t=t_{m}}=M_{2 m}\left(y\left(t_{m}\right)\right), m=1,2, \ldots, n, \\
\left.\Delta y^{\prime}\right|_{t=t_{m}}=N_{2 m}\left(y\left(t_{m}\right)\right), m=1,2, \ldots, n, \\
\left.\Delta y^{\prime \prime}\right|_{t=t_{m}}=O_{2 m}\left(y\left(t_{m}\right)\right), m=1,2, \ldots, n, \\
y(0)=y^{\prime}(0)=0,{ }^{c} D_{0^{+}}^{\rho} y(\Phi)=y^{\prime \prime}(1), 0<\rho, \Phi<1,
\end{array}\right.
$$

if and only if $\mathrm{y} \in \mathrm{PC}(\mathrm{J})$ is the solution of the integral equation

$$
y(t)=\left\{\begin{array}{l}
-\frac{1}{\Gamma(\beta)} \int_{0}^{t}(t-s)^{\beta-1} \vartheta(s) d s+c^{*} t^{2}, t \in\left[0, t_{1}\right] \\
-\frac{1}{\Gamma(\beta)} \int_{t_{m}}^{t}(t-s)^{\beta-1} \vartheta(s) d s-\frac{1}{\Gamma(\beta)} \sum_{j=1}^{m} \int_{t_{j-1}}^{t_{j}}\left(t_{j}-s\right)^{\beta-1} \vartheta(s) d s \\
-\frac{1}{\Gamma(\beta-1)} \sum_{j=1}^{m}\left(t-t_{j}\right) \int_{t_{j-1}}^{t_{j}}\left(t_{j}-s\right)^{\beta-2} \vartheta(s) d s-\frac{1}{2 \Gamma(\beta-2)} \sum_{j=1}^{m}\left(t-t_{j}\right)^{2} \\
\times \int_{t_{j-1}}^{t_{j}}\left(t_{j}-s\right)^{\beta-3} \vartheta(s) d s+\sum_{j=1}^{m} M_{2 j}\left(y\left(t_{j}\right)\right)+\sum_{j=1}^{m}\left(t-t_{j}\right) N_{2 j}\left(y\left(t_{j}\right)\right) \\
+\sum_{j=1}^{m} \frac{\left(t-t_{j}\right)^{2}}{2} O_{2 j}\left(y\left(t_{j}\right)\right)+c^{*} t^{2}, t \in\left(t_{m}, t_{m+1}\right], 1 \leqslant m \leqslant n
\end{array}\right.
$$

where $c \in\{0,1, \ldots, n\}$ such that $\Phi \in\left(\mathfrak{t}_{\mathfrak{c}}, \mathbf{t}_{\mathfrak{c}+1}\right]$, and 


$$
\begin{aligned}
c^{*}= & \frac{\Gamma(3-\rho)}{2\left(\Gamma(3-\rho)-\Phi^{2-\rho)}\right.}\left[-\frac{1}{\Gamma(\beta-\rho)} \int_{t_{j}}^{\Phi}(\Phi-s)^{\beta-\rho-1} \vartheta(s) d s-\frac{\Phi^{1-\rho}}{\Gamma(\beta-1) \Gamma(2-\rho)}\right. \\
& \times \sum_{j=1}^{m} \int_{t_{j-1}}^{t_{j}}\left(t_{j}-s\right)^{\beta-2} \vartheta(s) d s-\frac{\Phi^{2-\rho}}{\Gamma(\beta-2) \Gamma(3-\rho)} \sum_{j=1}^{m} \int_{t_{j-1}}^{t_{j}}\left(t_{j}-s\right)^{\beta-3} \vartheta(s) d s \\
& +\frac{\Phi^{1-\rho}}{\Gamma(\beta-2) \Gamma(2-\rho)} \sum_{j=1}^{m} t_{j} \int_{t_{j-1}}^{t_{j}}\left(t_{j}-s\right)^{\beta-3} \vartheta(s) d s+\frac{\Phi^{1-\rho}}{\Gamma(2-\rho)} \sum_{j=1}^{m} N_{2 j}\left(y\left(t_{j}\right)\right) \\
& +\frac{\Phi^{2-\rho}}{\Gamma(3-\rho)} \sum_{j=1}^{m} O_{2 j}\left(y\left(t_{j}\right)\right)-\frac{\Phi^{1-\rho}}{\Gamma(2-\rho)} \sum_{j=1}^{m} t_{j} O_{2 j}\left(y\left(t_{j}\right)\right)+\frac{1}{\Gamma(\beta-2)} \int_{t_{m}}^{1}(1-s)^{\beta-3} \vartheta(s) d s \\
& \left.+\frac{1}{\Gamma(\beta-2)} \sum_{j=1}^{m} \int_{t_{j-1}}^{t_{j}}\left(t_{j}-s\right)^{\beta-3} \vartheta(s) d s-\sum_{j=1}^{m} O_{2 j}\left(y\left(t_{j}\right)\right)\right] .
\end{aligned}
$$

\section{Main Results}

In this section, we use fixed point theorems to prove the existence of solutions to problem (1). According to Lemmas 4 and 5, we define operator $\mathbf{W}: Y^{\prime} \longrightarrow Y^{\prime}$ by

$$
\mathbf{W}(x, y)(t)=\left(\mathbf{W}_{1}(x, y)(t), \mathbf{W}_{2}(x, y)(t)\right)^{\top}, \forall(x, y) \in Y^{\prime}, t \in[0,1]
$$

where

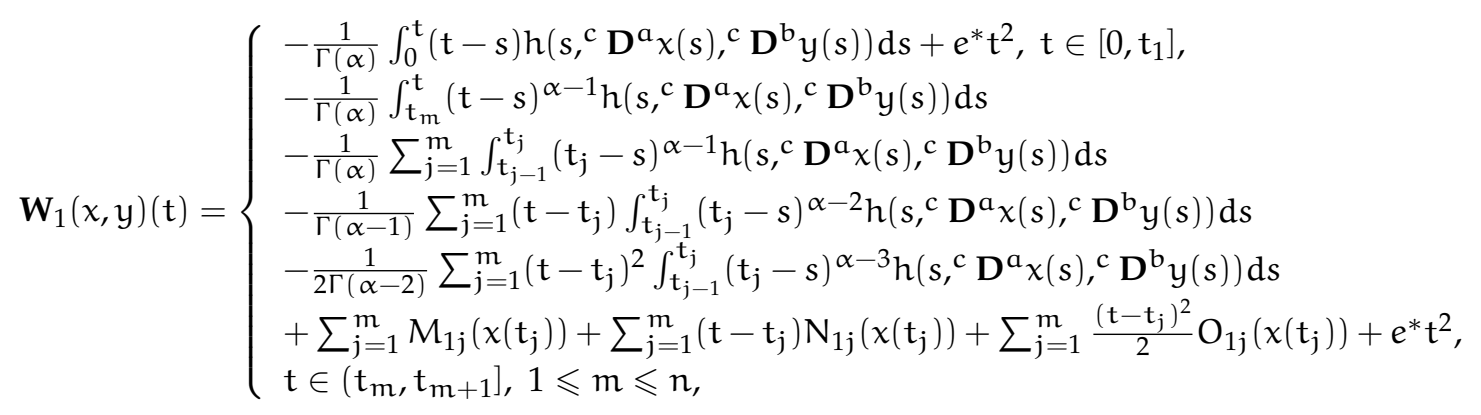

and

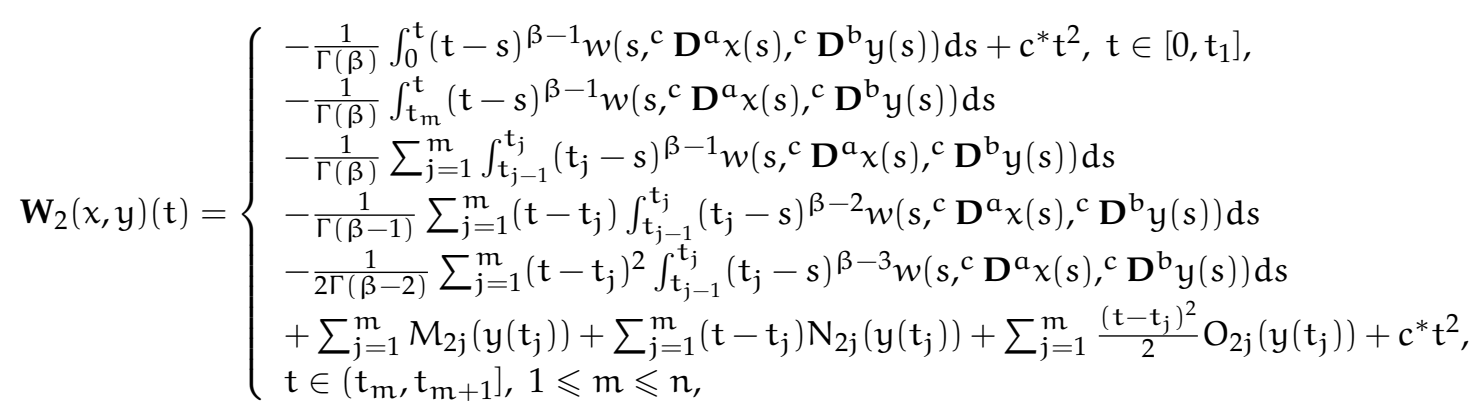

with

$$
\begin{aligned}
e^{*}= & z_{\alpha}\left[-\frac{1}{\Gamma(\alpha-\varepsilon)} \int_{t_{j}}^{\Omega}(\Omega-s)^{\alpha-\varepsilon-1} h\left(s^{c}{ }^{c} D^{a} x(s),{ }^{c} D^{b} y(s)\right) d s\right. \\
& -\frac{\Omega^{1-\varepsilon}}{\Gamma(\alpha-1) \Gamma(2-\varepsilon)} \sum_{j=1}^{m} \int_{t_{j-1}}^{t_{j}}\left(t_{j}-s\right)^{\alpha-2} h\left(s,{ }^{c} D^{a} x(s),{ }^{c} D^{b} y(s)\right) d s \\
& -\frac{\Omega^{2-\varepsilon}}{\Gamma(\alpha-2) \Gamma(3-\varepsilon)} \sum_{j=1}^{m} \int_{t_{j-1}}^{t_{j}}\left(t_{j}-s\right)^{\alpha-3} h\left(s,{ }^{c} D^{a} x(s),{ }^{c} D^{b} y(s)\right) d s
\end{aligned}
$$




$$
\begin{aligned}
& +\frac{\Omega^{1-\varepsilon}}{\Gamma(\alpha-2) \Gamma(2-\varepsilon)} \sum_{j=1}^{m} t_{j} \int_{t_{j-1}}^{t_{j}}\left(t_{j}-s\right)^{\alpha-3} h\left(s,{ }^{c} D^{a} x(s),{ }^{c} D^{b} y(s)\right) d s \\
& +\frac{\Omega^{1-\varepsilon}}{\Gamma(2-\varepsilon)} \sum_{j=1}^{m} N_{1 j}\left(x\left(t_{j}\right)\right)+\frac{\Omega^{2-\varepsilon}}{\Gamma(3-\varepsilon)} \sum_{j=1}^{m} O_{1 j}\left(x\left(t_{j}\right)\right)-\frac{\Omega^{1-\varepsilon}}{\Gamma(2-\varepsilon)} \sum_{j=1}^{m} t_{j} O_{1 j}\left(x\left(t_{j}\right)\right) \\
& +\frac{1}{\Gamma(\alpha-2)} \int_{t_{m}}^{1}(1-s)^{\alpha-3} h\left(s,{ }^{c} D^{a} x(s),{ }^{c} D^{b} y(s)\right) d s \\
& \left.+\frac{1}{\Gamma(\alpha-2)} \sum_{j=1}^{m} \int_{t_{j-1}}^{t_{j}}\left(t_{j}-s\right)^{\alpha-3} h\left(s^{c}{ }^{c} D^{a} \chi(s),{ }^{c} D^{b} y(s)\right) d s-\sum_{j=1}^{m} O_{1 j}\left(x\left(t_{j}\right)\right)\right], \\
& c^{*}=z_{\beta}\left[-\frac{1}{\Gamma(\beta-\rho)} \int_{t_{j}}^{\Phi}(\Phi-s)^{\beta-\rho-1} \mathcal{w}\left(s^{c}{ }^{c} D^{a} x(s),{ }^{c} D^{b} y(s)\right) d s\right. \\
& -\frac{\Phi^{1-\rho}}{\Gamma(\beta-1) \Gamma(2-\rho)} \sum_{j=1}^{m} \int_{t_{j-1}}^{t_{j}}\left(t_{j}-s\right)^{\beta-2} w\left(s,{ }^{c} D^{a} x(s),{ }^{c} D^{b} y(s)\right) d s \\
& -\frac{\Phi^{2-\rho}}{\Gamma(\beta-2) \Gamma(3-\rho)} \sum_{j=1}^{\mathrm{m}} \int_{\mathrm{t}_{j-1}}^{\mathrm{t}_{j}}\left(\mathrm{t}_{j}-s\right)^{\beta-3} w\left(s^{\mathrm{c}}{ }^{\mathrm{c}} \mathbf{D}^{\mathrm{a}} x(\mathrm{~s}),{ }^{\mathrm{c}} \mathbf{D}^{\mathrm{b}} \mathrm{y}(\mathrm{s})\right) \mathrm{d} s \\
& \left.+\frac{\Phi^{1-\rho}}{\Gamma(\beta-2) \Gamma(2-\rho)} \sum_{j=1}^{m} t_{j} \int_{t_{j-1}}^{t_{j}}\left(t_{j}-s\right)^{\beta-3} w\left(s,{ }^{c} D^{a} x(s),{ }^{c} D^{b} y(s)\right) d s\right) \\
& +\frac{\Phi^{1-\rho}}{\Gamma(2-\rho)} \sum_{j=1}^{m} N_{2 j}\left(y\left(t_{j}\right)+\frac{\Phi^{2-\rho}}{\Gamma(3-\rho)} \sum_{j=1}^{m} O_{2 j}\left(y\left(t_{j}\right)\right)-\frac{\Phi^{1-\rho}}{\Gamma(2-\rho)} \sum_{j=1}^{m} t_{j} O_{2 j}\left(y\left(t_{j}\right)\right)\right. \\
& +\frac{1}{\Gamma(\beta-2)} \int_{\mathbf{t}_{\mathrm{m}}}^{1}(1-\mathrm{s})^{\beta-3} w\left(\mathrm{~s}^{\mathrm{c}}, \mathbf{D}^{\mathrm{a}} x(\mathrm{~s}),{ }^{\mathrm{c}} \mathbf{D}^{\mathrm{b}} \mathrm{y}(\mathrm{s})\right) \mathrm{d} \mathrm{s} \\
& \left.+\frac{1}{\Gamma(\beta-2)} \sum_{j=1}^{m} \int_{t_{j-1}}^{t_{j}}\left(t_{j}-s\right)^{\beta-3} w\left(s,{ }^{c} D^{a} x(s),{ }^{c} D^{b} y(s)\right) d s-\sum_{j=1}^{m} O_{2 j}\left(y\left(t_{j}\right)\right)\right],
\end{aligned}
$$

where $z_{\alpha}=\frac{\Gamma(3-\varepsilon)}{2\left(\Gamma(3-\varepsilon)-\Omega^{2-\varepsilon}\right)}$ and $z_{\beta}=\frac{\Gamma(3-\rho)}{2\left(\Gamma(3-\rho)-\Phi^{2-\rho}\right)}$. Thus, solving problem (1) is equivalent to obtain a fixed point of the operator $\mathbf{W}$. Next, we have to prove the uniqueness of solutions of problem (1).

Theorem 2. Let the following conditions $\left(\mathbf{M}_{1}\right)-\left(\mathbf{M}_{3}\right)$ hold, and then the boundary value problem (1) has a unique solution.

$\left(\mathbf{M}_{1}\right)$ : For all $\mathrm{t} \in \mathrm{J}$ and $\mathrm{x}_{\mathbf{j}}, \mathrm{y}_{\mathbf{j}} \in \mathbb{R}(\mathbf{j}=1,2)$ there exists some positive constants $\mu_{j}, \mu_{j}^{\prime}(j=1,2)$ such that

$$
\begin{aligned}
& \left|h\left(t, x_{1}, y_{1}\right)-h\left(t, x_{2}, y_{2}\right)\right| \leqslant \mu_{1}\left|x_{1}-x_{2}\right|+\mu_{2}\left|y_{1}-y_{2}\right| \\
& \left|w\left(t, x_{1}, y_{1}\right)-w\left(t, x_{2}, y_{2}\right)\right| \leqslant \mu_{1}^{\prime}\left|x_{1}-x_{2}\right|+\mu_{2}^{\prime}\left|y_{1}-y_{2}\right| .
\end{aligned}
$$

$\left(\mathbf{M}_{2}\right):$ For all $x, y \in \mathbb{R}$, there exist some positive constants $\mathrm{I}_{j k}, \hat{\mathrm{I}}_{j k}, \breve{I}_{j k}(j=1,2 ; m=1,2, \ldots, n)$ such that

$$
\begin{array}{r}
\left|M_{j m}(x)-M_{j m}(y)\right| \leqslant I_{j m}|x-y|, \\
\left|N_{j m}(x)-N_{j m}(y)\right| \leqslant \hat{I}_{j m}|x-y|, \\
\left|O_{j m}(x)-O_{j m}(y)\right| \leqslant \check{I}_{j m}|x-y| .
\end{array}
$$


$\left(\mathbf{M}_{3}\right)$ :

$$
\begin{aligned}
& {\left[( \mu _ { 1 } + \mu _ { 2 } ) \left(\frac{2}{\Gamma(\alpha+1)}+\frac{1}{\Gamma(\alpha)}+\frac{1}{2 \Gamma(\alpha-1)}+\frac{z_{\alpha}}{\Gamma(\alpha-\varepsilon+1)}+\frac{z_{\alpha} \Omega^{1-\varepsilon}}{\Gamma(\alpha) \Gamma(2-\varepsilon)}+\frac{z_{\alpha} \Omega^{2-\varepsilon}}{\Gamma(\alpha-1) \Gamma(3-\varepsilon)}\right.\right.} \\
& \left.+\frac{z_{\alpha} \Omega^{1-\varepsilon}}{\Gamma(\alpha-1) \Gamma(2-\varepsilon)}+\frac{2 z_{\alpha}}{\Gamma(\alpha-1)}\right)+\sum_{j=1}^{m} \mathrm{I}_{1 j}+\sum_{j=1}^{m} \hat{\mathrm{I}}_{1 j}+\frac{z_{\alpha} \Omega^{1-\varepsilon}}{\Gamma(2-\varepsilon)} \sum_{j=1}^{m} \hat{\mathrm{I}}_{1 j}+\frac{z_{\alpha} \Omega^{2-\varepsilon}}{\Gamma(3-\varepsilon)} \sum_{j=1}^{m} \check{\mathrm{I}}_{1 j} \\
& \left.+\frac{z_{\alpha} \Omega^{1-\varepsilon}}{\Gamma(2-\varepsilon)} \sum_{j=1}^{m} \check{\mathrm{I}}_{1 j}+z_{\alpha} \sum_{j=1}^{m} \check{\mathrm{I}}_{1 j}+\frac{1}{2} \sum_{j=1}^{m} \breve{\mathrm{I}}_{1 j}\right]<1
\end{aligned}
$$

and

$$
\begin{aligned}
& {\left[( \mu _ { 1 } ^ { \prime } + \mu _ { 2 } ^ { \prime } ) \left(\frac{2}{\Gamma(\beta+1)}+\frac{1}{\Gamma(\beta)}+\frac{1}{2 \Gamma(\beta-1)}+\frac{z_{\beta}}{\Gamma(\beta-\rho+1)}+\frac{z_{\beta} \Phi^{1-\rho}}{\Gamma(\beta) \Gamma(2-\rho)}+\frac{z_{\beta} \Phi^{2-\rho}}{\Gamma(\beta-1) \Gamma(3-\rho)}\right.\right.} \\
& \left.+\frac{z_{\beta} \Phi^{1-\rho}}{\Gamma(\beta-1) \Gamma(2-\rho)}+\frac{2 z_{\beta}}{\Gamma(\beta-1)}\right)+\sum_{j=1}^{m} \mathrm{I}_{2 j}+\sum_{j=1}^{m} \hat{\mathrm{I}}_{2 j}+\frac{z_{\beta} \Phi^{1-\rho}}{\Gamma(2-\rho)} \sum_{j=1}^{m} \hat{\mathrm{I}}_{2 j}+\frac{z_{\beta} \Phi^{2-\rho}}{\Gamma(3-\rho)} \sum_{j=1}^{m} \check{\mathrm{I}}_{2 j} \\
& \left.+\frac{z_{\beta} \Phi^{1-\rho}}{\Gamma(2-\rho)} \sum_{j=1}^{m} \breve{\mathrm{I}}_{2 j}+z_{\beta} \sum_{j=1}^{m} \check{\mathrm{I}}_{2 j}+\frac{1}{2} \sum_{j=1}^{m} \breve{\mathrm{I}}_{2 j}\right]<1 .
\end{aligned}
$$

Proof. By using the Banach contraction principle, we can prove that $\mathbf{W}$, defined by (17), has a fixed point. Before proving the main result first, we will prove the contraction. When $t \in J$, from (17) and conditions $\left(\mathbf{M}_{1}\right)-\left(\mathbf{M}_{2}\right)$, for all $\left(x_{1}, y_{1}\right),\left(x_{2}, y_{2}\right) \in Y^{\prime}$, we have

$$
\begin{aligned}
& \left|\mathbf{W}_{1}\left(\mathrm{x}_{1}, \mathrm{y}_{1}\right)(\mathrm{t})-\mathbf{W}_{1}\left(\mathrm{x}_{2}, \mathrm{y}_{2}\right)(\mathrm{t})\right| \\
& \leqslant \frac{1}{\Gamma(\alpha)} \int_{0}^{t}(t-s)^{\alpha-1}\left|h\left(s,{ }^{c} D^{a} x_{1}(s),{ }^{c} D^{b} y_{1}(s)\right)-h\left(s,{ }^{c} D^{a} x_{2}(s),{ }^{c} D^{b} y_{2}(s)\right)\right| d s \\
& +\frac{z_{\alpha} t^{2}}{\Gamma(\alpha-\varepsilon)} \int_{t_{j}}^{\Omega}(\Omega-s)^{\alpha-\varepsilon-1}\left|h\left(s,{ }^{c} D^{a} x_{1}(s),{ }^{c} D^{b} y_{1}(s)\right)-h\left(s,{ }^{c} D^{a} x_{2}(s),{ }^{c} D^{b} y_{2}(s)\right)\right| d s \\
& +\frac{z_{\alpha} t^{2} \Omega^{1-\varepsilon}}{\Gamma(\alpha-1) \Gamma(2-\varepsilon)} \sum_{j=1}^{m} \int_{t_{j-1}}^{t_{j}}\left(t_{j}-s\right)^{\alpha-2}\left|h\left(s,{ }^{c} D^{a} x_{1}(s),{ }^{c} D^{b} y_{1}(s)\right)-h\left(s,{ }^{c} D^{a} x_{2}(s),{ }^{c} D^{b} y_{2}(s)\right)\right| d s \\
& +\frac{z_{\alpha} t^{2} \Omega^{2-\varepsilon}}{\Gamma(\alpha-2) \Gamma(3-\varepsilon)} \sum_{j=1}^{m} \int_{t_{j-1}}^{t_{j}}\left(t_{j}-s\right)^{\alpha-3}\left|h\left(s,{ }^{c} D^{a} x_{1}(s),{ }^{c} D^{b} y_{1}(s)\right)-h\left(s,{ }^{c} D^{a} x_{2}(s),{ }^{c} D^{b} y_{2}(s)\right)\right| d s \\
& +\frac{z_{\alpha} t^{2} \Omega^{1-\varepsilon}}{\Gamma(\alpha-2) \Gamma(2-\varepsilon)} \sum_{j=1}^{m} t_{j} \int_{t_{j-1}}^{t_{j}}\left(t_{j}-s\right)^{\alpha-3}\left|h\left(s,{ }^{c} D^{a} x_{1}(s),{ }^{c} D^{b} y_{1}(s)\right)-h\left(s,{ }^{c} D^{a} x_{2}(s),{ }^{c} D^{b} y_{2}(s)\right)\right| d s \\
& +\frac{z_{\alpha} t^{2} \Omega^{1-\varepsilon}}{\Gamma(2-\varepsilon)} \sum_{j=1}^{m}\left|N_{1 j}\left(x_{1}\left(t_{j}\right)\right)-N_{1 j}\left(x_{2}\left(t_{j}\right)\right)\right|+\frac{z_{\alpha} t^{2} \Omega^{2-\varepsilon}}{\Gamma(3-\varepsilon)} \sum_{j=1}^{m}\left|O_{1 j}\left(x_{1}\left(t_{j}\right)\right)-O_{1 j}\left(x_{2}\left(t_{j}\right)\right)\right| \\
& +\frac{z_{\alpha} \mathrm{t}^{2}}{\Gamma(\alpha-2)} \int_{\mathrm{t}_{\mathrm{m}}}^{1}(1-\mathrm{s})^{\alpha-3}\left|\mathrm{~h}\left(\mathrm{~s}^{\mathrm{c}}, \mathbf{D}^{\mathrm{a}} \mathrm{x}_{1}(\mathrm{~s}),{ }^{\mathrm{c}} \mathbf{D}^{\mathrm{b}} \mathrm{y}_{1}(\mathrm{~s})\right)-\mathrm{h}\left(\mathrm{s}^{\mathrm{c}}{ }^{\mathrm{c}} \mathbf{D}^{\mathrm{a}} \mathrm{x}_{2}(\mathrm{~s}),{ }^{\mathrm{c}} \mathbf{D}^{\mathrm{b}} \mathrm{y}_{2}(\mathrm{~s})\right)\right| \mathrm{ds} \\
& +\frac{z_{\alpha} t^{2}}{\Gamma(\alpha-2)} \sum_{j=1}^{m} \int_{t_{j-1}}^{t_{j}}\left(t_{j}-s\right)^{\alpha-3}\left|h\left(s,{ }^{c} D^{a} x_{1}(s),{ }^{c} D^{b} y_{1}(s)\right)-h\left(s,{ }^{c} D^{a} x_{2}(s),{ }^{c} D^{b} y_{2}(s)\right)\right| d s \\
& +\frac{z_{\alpha} t^{2} \Omega^{1-\varepsilon}}{\Gamma(2-\varepsilon)} \sum_{j=1}^{m}\left|t_{j}\right|\left|O_{1 j}\left(x_{1}\left(t_{j}\right)\right)-O_{1 j}\left(x_{2}\left(t_{j}\right)\right)\right|+z_{\alpha} t^{2} \sum_{j=1}^{m}\left|O_{1 j}\left(x_{1}\left(t_{j}\right)\right)-O_{1 j}\left(x_{2}\left(t_{j}\right)\right)\right|, \\
& \leqslant \frac{1}{\Gamma(\alpha+1)}\left[\mu_{1}\left|{ }^{c} D^{a} x_{1}(s)-{ }^{c} D^{a} x_{2}(s)\right|+\mu_{2}\left|{ }^{c} D^{b} y_{1}(s)-{ }^{c} D^{b} y_{2}(s)\right|\right]
\end{aligned}
$$




$$
\begin{aligned}
& +\frac{z_{\alpha}}{\Gamma(\alpha-\varepsilon+1)}\left[\mu_{1}\left|{ }^{\mathrm{c}} \mathbf{D}^{\mathrm{a}} x_{1}(\mathrm{~s})-{ }^{\mathrm{c}} \mathbf{D}^{\mathrm{a}} x_{2}(\mathrm{~s})\right|+\mu_{2}\left|{ }^{\mathrm{c}} \mathbf{D}^{\mathrm{b}} \mathbf{y}_{1}(\mathrm{~s})-{ }^{\mathrm{c}} \mathbf{D}^{\mathrm{b}} \mathbf{y}_{2}(\mathrm{~s})\right|\right] \\
& +\frac{z_{\alpha} \Omega^{1-\varepsilon}}{\Gamma(\alpha) \Gamma(2-\varepsilon)}\left[\mu_{1}\left|{ }^{c} D^{a} x_{1}(s)-{ }^{c} D^{a} x_{2}(s)\right|+\mu_{2}\left|{ }^{c} D^{b} y_{1}(s)-{ }^{c} D^{b} y_{2}(s)\right|\right] \\
& +\frac{z_{\alpha} \Omega^{2-\varepsilon}}{\Gamma(\alpha-1) \Gamma(3-\varepsilon)}\left[\mu_{1}\left|{ }^{c} D^{a} x_{1}(s)-{ }^{c} D^{a} x_{2}(s)\right|+\mu_{2}\left|{ }^{c} D^{a} y_{1}(s)-{ }^{c} D^{a} y_{2}(s)\right|\right] \\
& +\frac{z_{\alpha} \Omega^{1-\varepsilon}}{\Gamma(\alpha-1) \Gamma(2-\varepsilon)}\left[\mu_{1}\left|{ }^{c} D^{a} x_{1}(s)-{ }^{c} D^{a} x_{2}(s)\right|+\mu_{2}\left|{ }^{c} D^{b} y_{1}(s)-{ }^{c} D^{b} y_{2}(s)\right|\right] \\
& +\frac{z_{\alpha} \Omega^{1-\varepsilon}}{\Gamma(2-\varepsilon)} \sum_{j=1}^{m}\left|N_{1 j}\left(x_{1}\left(t_{j}\right)\right)-N_{1 j}\left(x_{2}\left(t_{j}\right)\right)\right|+\frac{z_{\alpha} \Omega^{2-\varepsilon}}{\Gamma(3-\varepsilon)} \sum_{j=1}^{m}\left|O_{1 j}\left(x_{1}\left(t_{j}\right)\right)-O_{1 j}\left(x_{2}\left(t_{j}\right)\right)\right| \\
& +\frac{z_{\alpha}}{\Gamma(\alpha-1)}\left[\mu_{1}\left|{ }^{c} \mathbf{D}^{\mathrm{a}} x_{1}(\mathrm{~s})-{ }^{\mathrm{c}} \mathbf{D}^{\mathrm{a}} \mathrm{x}_{2}(\mathrm{~s})\right|+\mu_{2}\left|{ }^{\mathrm{c}} \mathbf{D}^{\mathrm{b}} \mathrm{y}_{1}(\mathrm{~s})-{ }^{\mathrm{c}} \mathbf{D}^{\mathrm{b}} \mathrm{y}_{2}(\mathrm{~s})\right|\right] \\
& +\frac{z_{\alpha}}{\Gamma(\alpha-1)}\left[\mu_{1}\left|{ }^{\mathrm{c}} \mathbf{D}^{\mathrm{a}} x_{1}(\mathrm{~s})-{ }^{\mathrm{c}} \mathbf{D}^{\mathrm{a}} x_{2}(\mathrm{~s})\right|+\mu_{2}\left|{ }^{\mathrm{c}} \mathbf{D}^{\mathrm{b}} \mathrm{y}_{1}(\mathrm{~s})-{ }^{\mathrm{c}} \mathbf{D}^{\mathrm{b}} \mathrm{y}_{2}(\mathrm{~s})\right|\right] \\
& +\frac{z_{\alpha} \Omega^{1-\varepsilon}}{\Gamma(2-\varepsilon)} \sum_{j=1}^{m} t_{j}\left|O_{1 j}\left(x_{1}\left(t_{j}\right)\right)-O_{1 j}\left(x_{2}\left(t_{j}\right)\right)\right|+z_{\alpha} \sum_{j=1}^{m}\left|O_{1 j}\left(x_{1}\left(t_{j}\right)\right)-O_{1 j}\left(x_{2}\left(t_{j}\right)\right)\right|, \\
& \leqslant \frac{1}{\Gamma(\alpha+1)}\left[\mu_{1}\left\|x_{1}-x_{2}\right\|_{0}+\mu_{2}\left\|y_{1}-y_{2}\right\|_{0}\right]+\frac{z_{\alpha}}{\Gamma(\alpha-\varepsilon+1)}\left[\mu_{1}\left\|x_{1}-x_{2}\right\|_{0}+\mu_{2}\left\|y_{1}-y_{2}\right\|_{0}\right] \\
& +\frac{z_{\alpha} \Omega^{1-\varepsilon}}{\Gamma(\alpha) \Gamma(2-\varepsilon)}\left[\mu_{1}\left\|x_{1}-x_{2}\right\|_{0}+\mu_{2}\left\|y_{1}-y_{2}\right\|_{0}\right]+\frac{z_{\alpha} \Omega^{2-\varepsilon}}{\Gamma(\alpha-1) \Gamma(3-\varepsilon)}\left[\mu_{1}\left\|x_{1}-x_{2}\right\|_{0}+\mu_{2}\left\|y_{1}-y_{2}\right\|_{0}\right] \\
& +\frac{z_{\alpha} \Omega^{1-\varepsilon}}{\Gamma(\alpha-1) \Gamma(2-\varepsilon)}\left[\mu_{1}\left\|x_{1}-x_{2}\right\|_{0}+\mu_{2}\left\|y_{1}-y_{2}\right\|_{0}\right]+\frac{z_{\alpha} \Omega^{1-\varepsilon}}{\Gamma(2-\varepsilon)} \sum_{j=1}^{m} \hat{I}_{1 j}\left\|x_{1}-x_{2}\right\|_{0}+\frac{z_{\alpha} \Omega^{2-\varepsilon}}{\Gamma(3-\varepsilon)} \sum_{j=1}^{m} \check{I}_{1 j} \\
& \times\left\|x_{1}-x_{2}\right\|_{0}+\frac{z_{\alpha}}{\Gamma(\alpha-1)}\left[\mu_{1}\left\|x_{1}-x_{2}\right\|_{0}+\mu_{2}\left\|y_{1}-y_{2}\right\|_{0}\right]+\frac{z_{\alpha}}{\Gamma(\alpha-1)}\left[\mu_{1}\left\|x_{1}-x_{2}\right\|_{0}+\mu_{2}\left\|y_{1}-y_{2}\right\|_{0}\right] \\
& +\frac{z_{\alpha} \Omega^{1-\varepsilon}}{\Gamma(2-\varepsilon)} \sum_{j=1}^{m} \breve{I}_{1 j}\left\|x_{1}-x_{2}\right\|_{0}+z_{\alpha} \sum_{j=1}^{m} \breve{I}_{1 j}\left\|x_{1}-x_{2}\right\|_{0} \\
& \leqslant \frac{1}{\Gamma(\alpha+1)}\left[\mu_{1}+\mu_{2}\right]\left\|\left(x_{1}-x_{2}, y_{1}-y_{2}\right)\right\|+\frac{z_{\alpha}}{\Gamma(\alpha-\varepsilon+1)}\left[\mu_{1}+\mu_{2}\right]\left\|\left(x_{1}-x_{2}, y_{1}-y_{2}\right)\right\| \\
& +\frac{z_{\alpha} \Omega^{1-\varepsilon}}{\Gamma(\alpha) \Gamma(2-\varepsilon)}\left[\mu_{1}+\mu_{2}\right]\left\|\left(x_{1}-x_{2}, y_{1}-y_{2}\right)\right\|+\frac{z_{\alpha} \Omega^{2-\varepsilon}}{\Gamma(\alpha-1) \Gamma(3-\varepsilon)}\left[\mu_{1}+\mu_{2}\right]\left\|\left(x_{1}-x_{2}, y_{1}-y_{2}\right)\right\| \\
& +\frac{z_{\alpha} \Omega^{1-\varepsilon}}{\Gamma(\alpha-1) \Gamma(2-\varepsilon)}\left[\mu_{1}+\mu_{2}\right]\left\|\left(x_{1}-x_{2}, y_{1}-y_{2}\right)\right\|+\frac{z_{\alpha} \Omega^{1-\varepsilon}}{\Gamma(2-\varepsilon)} \sum_{j=1}^{m} \hat{I}_{1 j}\left\|\left(x_{1}-x_{2}, y_{1}-y_{2}\right)\right\| \\
& +\frac{z_{\alpha} \Omega^{2-\varepsilon}}{\Gamma(3-\varepsilon)} \sum_{j=1}^{m} \check{I}_{1 j}\left\|\left(x_{1}-x_{2}, y_{1}-y_{2}\right)\right\|+\frac{z_{\alpha}}{\Gamma(\alpha-1)}\left[\mu_{1}+\mu_{2}\right]\left\|\left(x_{1}-x_{2}, y_{1}-y_{2}\right)\right\| \\
& +\frac{z_{\alpha}}{\Gamma(\alpha-1)}\left[\mu_{1}+\mu_{2}\right]\left\|\left(x_{1}-x_{2}, y_{1}-y_{2}\right)\right\|+\frac{z_{\alpha} \Omega^{1-\varepsilon}}{\Gamma(2-\varepsilon)} \sum_{j=1}^{m} \breve{I}_{1 j}\left\|\left(x_{1}-x_{2}, y_{1}-y_{2}\right)\right\| \\
& +z_{\alpha} \sum_{j=1}^{m} \check{I}_{1 j}\left\|\left(x_{1}-x_{2}, y_{1}-y_{2}\right)\right\| \\
& \leqslant z_{\alpha}\left[( \mu _ { 1 } + \mu _ { 2 } ) \left(\frac{1}{z_{\alpha} \Gamma(\alpha+1)}+\frac{1}{\Gamma(\alpha-\varepsilon+1)}+\frac{\Omega^{1-\varepsilon}}{\Gamma(\alpha) \Gamma(2-\varepsilon)}+\frac{\Omega^{2-\varepsilon}}{\Gamma(\alpha-1) \Gamma(3-\varepsilon)}+\frac{\Omega^{1-\varepsilon}}{\Gamma(\alpha-1) \Gamma(2-\varepsilon)}\right.\right. \\
& \left.\left.+\frac{2}{\Gamma(\alpha-1)}\right)+\frac{\Omega^{1-\varepsilon}}{\Gamma(2-\varepsilon)} \sum_{j=1}^{m} \hat{\mathrm{I}}_{1 j}+\frac{\Omega^{2-\varepsilon}}{\Gamma(3-\varepsilon)} \sum_{j=1}^{m} \breve{\mathrm{I}}_{1 j}+\frac{\Omega^{1-\varepsilon}}{\Gamma(2-\varepsilon)} \sum_{j=1}^{m} \check{\mathrm{I}}_{1 j}+\sum_{j=1}^{\mathrm{m}} \check{\mathrm{I}}_{1 j}\right] \\
& \times\left\|\left(x_{1}-x_{2}, y_{1}-y_{2}\right)\right\|, t \in\left[0, t_{1}\right] \text {. }
\end{aligned}
$$


When $t \in\left(t_{m}, t_{m+1}\right]$, then

$$
\begin{aligned}
& \left|\mathbf{W}_{1}\left(\mathrm{x}_{1}, \mathrm{y}_{1}\right)(\mathrm{t})-\mathbf{W}_{1}\left(\mathrm{x}_{2}, \mathrm{y}_{2}\right)(\mathrm{t})\right| \\
& \leqslant \frac{1}{\Gamma(\alpha)} \int_{\mathbf{t}_{\mathrm{m}}}^{\mathrm{t}}(\mathrm{t}-\mathrm{s})^{\alpha-1}\left|\mathrm{~h}\left(s^{\mathrm{c}}{ }^{\mathrm{c}} \mathbf{D}^{\mathrm{a}} \mathrm{x}_{1}(\mathrm{~s}),{ }^{\mathrm{c}} \mathbf{D}^{\mathrm{b}} \mathrm{y}_{1}(\mathrm{~s})\right)-\mathrm{h}\left(\mathrm{s}^{\mathrm{c}}, \mathbf{D}^{\mathrm{a}} \mathrm{x}_{2}(\mathrm{~s}),{ }^{\mathrm{c}} \mathbf{D}^{\mathrm{b}} \mathrm{y}_{2}(\mathrm{~s})\right)\right| \mathrm{d} \mathrm{s} \\
& +\frac{1}{\Gamma(\alpha)} \sum_{j=1}^{m} \int_{t_{j-1}}^{t_{j}}\left(t_{j}-s\right)^{\alpha-1}\left|h\left(s,{ }^{c} D^{a} x_{1}(s),{ }^{c} D^{b} y_{1}(s)\right)-h\left(s,{ }^{c} D^{a} x_{2}(s),{ }^{c} D^{b} y_{2}(s)\right)\right| d s \\
& +\frac{1}{\Gamma(\alpha-1)} \sum_{j=1}^{m}\left|\left(t-t_{j}\right)\right| \int_{t_{j-1}}^{t_{j}}\left(t_{j}-s\right)^{\alpha-2}\left|h\left(s,{ }^{c} D^{a} x_{1}(s),{ }^{c} D^{b} y_{1}(s)\right)-h\left(s,{ }^{c} D^{a} x_{2}(s),{ }^{c} D^{b} y_{2}(s)\right)\right| d s \\
& +\frac{1}{2 \Gamma(\alpha-2)} \sum_{j=1}^{m}\left|\left(t-t_{j}\right)^{2}\right| \int_{t_{j-1}}^{t_{j}}\left(t_{j}-s\right)^{\alpha-3}\left|h\left(s,{ }^{c} D^{a} x_{1}(s),{ }^{c} D^{b} y_{1}(s)\right)-h\left(s,{ }^{c} D^{a} x_{2}(s),{ }^{c} D^{b} y_{2}(s)\right)\right| d s \\
& +\sum_{j=1}^{m}\left|M_{1 j}\left(x_{1}\left(t_{j}\right)\right)-M_{1 j}\left(x_{2}\left(t_{j}\right)\right)\right|+\sum_{j=1}^{m}\left|\left(t-t_{j}\right)\right|\left|N_{1 j}\left(x_{1}\left(t_{j}\right)\right)-N_{1 j}\left(x_{2}\left(t_{j}\right)\right)\right| \\
& +\frac{z_{\alpha} t^{2}}{\Gamma(\alpha-\varepsilon)} \int_{t_{j}}^{\Omega}(\Omega-s)^{\alpha-\varepsilon-1}\left|h\left(s,{ }^{c} D^{a} x_{1}(s),{ }^{c} D^{b} y_{1}(s)\right)-h\left(s,{ }^{c} D^{a} x_{2}(s),{ }^{c} D^{b} y_{2}(s)\right)\right| d s \\
& +\frac{z_{\alpha} t^{2} \Omega^{1-\varepsilon}}{\Gamma(\alpha-1) \Gamma(2-\varepsilon)} \sum_{j=1}^{m} \int_{t_{j-1}}^{t_{j}}\left(t_{j}-s\right)^{\alpha-2}\left|h\left(s,{ }^{c} D^{a} x_{1}(s),{ }^{c} D^{b} y_{1}(s)\right)-h\left(s,{ }^{c} D^{a} x_{2}(s),{ }^{c} D^{b} y_{2}(s)\right)\right| d s \quad \text { (19) } \\
& +\frac{z_{\alpha} \mathrm{t}^{2} \Omega^{2-\varepsilon}}{\Gamma(\alpha-2) \Gamma(3-\varepsilon)} \sum_{j=1}^{\mathrm{m}} \int_{\mathrm{t}_{j-1}}^{\mathrm{t}_{j}}\left(\mathrm{t}_{j}-\mathrm{s}\right)^{\alpha-3}\left|\mathrm{~h}\left(\mathrm{~s}^{\mathrm{c}}{ }^{\mathrm{c}} \mathbf{D}^{\mathrm{a}} \mathrm{x}_{1}(\mathrm{~s}),{ }^{\mathrm{c}} \mathbf{D}^{\mathrm{b}} \mathrm{y}_{1}(\mathrm{~s})\right)-\mathrm{h}\left(\mathrm{s}^{\mathrm{c}}{ }^{\mathrm{c}} \mathbf{D}^{\mathrm{a}} \mathrm{x}_{2}(\mathrm{~s}),{ }^{\mathrm{c}} \mathbf{D}^{\mathrm{b}} \mathrm{y}_{2}(\mathrm{~s})\right)\right| \mathrm{d} s \\
& +\frac{z_{\alpha} t^{2} \Omega^{1-\varepsilon}}{\Gamma(\alpha-2) \Gamma(2-\varepsilon)} \sum_{j=1}^{m}\left|t_{j}\right| \int_{t_{j-1}}^{t_{j}}\left(t_{j}-s\right)^{\alpha-3}\left|h\left(s,{ }^{c} D^{a} x_{1}(s),{ }^{c} D^{b} y_{1}(s)\right)-h\left(s,{ }^{c} D^{a} x_{2}(s),{ }^{c} D^{b} y_{2}(s)\right)\right| d s \\
& +\frac{z_{\alpha} t^{2} \Omega^{1-\varepsilon}}{\Gamma(2-\varepsilon)} \sum_{j=1}^{m}\left|N_{1 j}\left(x_{1}\left(t_{j}\right)\right)-N_{1 j}\left(x_{2}\left(t_{j}\right)\right)\right|+\frac{z_{\alpha} t^{2} \Omega^{2-\varepsilon}}{\Gamma(3-\varepsilon)} \sum_{j=1}^{m}\left|O_{1 j}\left(x_{1}\left(t_{j}\right)\right)-O_{1 j}\left(x_{2}\left(t_{j}\right)\right)\right| \\
& +\frac{z_{\alpha} \mathrm{t}^{2}}{\Gamma(\alpha-2)} \int_{\mathrm{t}_{\mathrm{m}}}^{1}(1-\mathrm{s})^{\alpha-3}\left|\mathrm{~h}\left(\mathrm{~s}^{\mathrm{c}} \mathbf{D}^{\mathrm{a}} \mathrm{x}_{1}(\mathrm{~s}),{ }_{,}^{\mathrm{c}} \mathbf{D}^{\mathrm{b}} \mathrm{y}_{1}(\mathrm{~s})\right)-\mathrm{h}\left(\mathrm{s}^{,}{ }^{\mathrm{c}} \mathbf{D}^{\mathrm{a}} \mathrm{x}_{2}(\mathrm{~s}),{ }^{\mathrm{c}} \mathbf{D}^{\mathrm{b}} \mathrm{y}_{2}(\mathrm{~s})\right)\right| \mathrm{d} \mathrm{s} \\
& +\frac{z_{\alpha} t^{2}}{\Gamma(\alpha-2)} \sum_{j=1}^{m} \int_{t_{j-1}}^{t_{j}}\left(t_{j}-s\right)^{\alpha-3}\left|h\left(s,{ }^{c} D^{a} x_{1}(s),{ }^{c} D^{b} y_{1}(s)\right)-h\left(s,{ }^{c} D^{a} x_{2}(s),{ }^{c} D^{b} y_{2}(s)\right)\right| d s \\
& +\frac{z_{\alpha} t^{2} \Omega^{1-\varepsilon}}{\Gamma(2-\varepsilon)} \sum_{j=1}^{m}\left|t_{j}\right|\left|O_{1 j}\left(x_{1}\left(t_{j}\right)\right)-O_{1 j}\left(x_{2}\left(t_{j}\right)\right)\right|+z_{\alpha} t^{2} \sum_{j=1}^{m}\left|O_{1 j}\left(x_{1}\left(t_{j}\right)\right)-O_{1 j}\left(x_{2}\left(t_{j}\right)\right)\right| \\
& +\sum_{j=1}^{m} \frac{\left|\left(t-t_{j}\right)^{2}\right|}{2}\left|O_{1 j}\left(x_{1}\left(t_{j}\right)\right)-O_{1 j}\left(x_{2}\left(t_{j}\right)\right)\right| \text {. }
\end{aligned}
$$

Utilizing $\left(\mathbf{M}_{1}\right)$ and $\left(\mathbf{M}_{2}\right)$ in (19) and taking the maximum, we get

$$
\begin{aligned}
\leqslant & \frac{1}{\Gamma(\alpha+1)}\left[\mu_{1}\left\|x_{1}-x_{2}\right\|_{0}+\mu_{2}\left\|y_{1}-y_{2}\right\|_{0}\right]+\frac{1}{\Gamma(\alpha+1)}\left[\mu_{1}\left\|x_{1}-x_{2}\right\|_{0}+\mu_{2}\left\|y_{1}-y_{2}\right\|_{0}\right] \\
& +\frac{1}{\Gamma(\alpha)}\left[\mu_{1}\left\|x_{1}-x_{2}\right\|_{0}+\mu_{2}\left\|y_{1}-y_{2}\right\|_{0}\right]+\frac{1}{2 \Gamma(\alpha-1)}\left[\mu_{1}\left\|x_{1}-x_{2}\right\|_{0}+\mu_{2}\left\|y_{1}-y_{2}\right\|_{0}\right] \\
& +\sum_{j=1}^{m} I_{1 j}\left\|x_{1}-x_{2}\right\|_{0}+\sum_{j=1}^{m} \hat{I}_{1 j}\left\|x_{1}-x_{2}\right\|_{0}+\frac{z_{\alpha}}{\Gamma(\alpha-\varepsilon+1)}\left[\mu_{1}\left\|x_{1}-x_{2}\right\|_{0}+\mu_{2}\left\|y_{1}-y_{2}\right\|_{0}\right] \\
& +\frac{z_{\alpha} \Omega^{1-\varepsilon}}{\Gamma(\alpha) \Gamma(2-\varepsilon)}\left[\mu_{1}\left\|x_{1}-x_{2}\right\|_{0}+\mu_{2}\left\|y_{1}-y_{2}\right\|_{0}\right]+\frac{z_{\alpha} \Omega^{2-\varepsilon}}{\Gamma(\alpha-1) \Gamma(3-\varepsilon)}\left[\mu_{1}\left\|x_{1}-x_{2}\right\|_{0}+\mu_{2}\left\|y_{1}-y_{2}\right\|_{0}\right]
\end{aligned}
$$




$$
\begin{aligned}
& +\frac{z_{\alpha} \Omega^{1-\varepsilon}}{\Gamma(\alpha-1) \Gamma(2-\varepsilon)}\left[\mu_{1}\left\|x_{1}-x_{2}\right\|_{0}+\mu_{2}\left\|y_{1}-y_{2}\right\|_{0}\right]+\frac{z_{\alpha} \Omega^{1-\varepsilon}}{\Gamma(2-\varepsilon)} \sum_{j=1}^{m} \hat{I}_{1 j}\left\|x_{1}-x_{2}\right\|_{0} \\
& +\frac{z_{\alpha} \Omega^{2-\varepsilon}}{\Gamma(3-\varepsilon)} \sum_{j=1}^{m} \check{I}_{1 j}\left\|x_{1}-x_{2}\right\|_{0}+\frac{z_{\alpha}}{\Gamma(\alpha-1)}\left[\mu_{1}\left\|x_{1}-x_{2}\right\|_{0}+\mu_{2}\left\|y_{1}-y_{2}\right\|_{0}\right] \\
& +\frac{z_{\alpha}}{\Gamma(\alpha-1)}\left[\mu_{1}\left\|x_{1}-x_{2}\right\|_{0}+\mu_{2}\left\|y_{1}-y_{2}\right\|_{0}\right]+\frac{z_{\alpha} \Omega^{1-\varepsilon}}{\Gamma(2-\varepsilon)} \sum_{j=1}^{m} \check{I}_{1 j}\left\|x_{1}-x_{2}\right\|_{0}+z_{\alpha} \sum_{j=1}^{m} \check{I}_{1 j}\left\|x_{1}-x_{2}\right\|_{0} \\
& +\frac{1}{2} \sum_{j=1}^{m} \check{I}_{1 j}\left\|x_{1}-x_{2}\right\|_{0} \\
& \leqslant \frac{1}{\Gamma(\alpha+1)}\left[\mu_{1}+\mu_{2}\right]\left\|\left(x_{1}-x_{2}, y_{1}-y_{2}\right)\right\|+\frac{1}{\Gamma(\alpha+1)}\left[\mu_{1}+\mu_{2}\right]\left\|\left(x_{1}-x_{2}, y_{1}-y_{2}\right)\right\| \\
& +\frac{1}{\Gamma(\alpha)}\left[\mu_{1}+\mu_{2}\right]\left\|\left(x_{1}-x_{2}, y_{1}-y_{2}\right)\right\|+\frac{1}{2 \Gamma(\alpha-1)}\left[\mu_{1}+\mu_{2}\right]\left\|\left(x_{1}-x_{2}, y_{1}-y_{2}\right)\right\| \\
& +\sum_{j=1}^{m} I_{1 j}\left\|\left(x_{1}-x_{2}, y_{1}-y_{2}\right)\right\|+\sum_{j=1}^{m} \hat{I}_{1 j}\left\|\left(x_{1}-x_{2}, y_{1}-y_{2}\right)\right\|+\frac{z_{\alpha}}{\Gamma(\alpha-\varepsilon+1)}\left[\mu_{1}+\mu_{2}\right]\left\|\left(x_{1}-x_{2}, y_{1}-y_{2}\right)\right\| \\
& +\frac{z_{\alpha} \Omega^{1-\varepsilon}}{\Gamma(\alpha) \Gamma(2-\varepsilon)}\left[\mu_{1}+\mu_{2}\right]\left\|\left(x_{1}-x_{2}, y_{1}-y_{2}\right)\right\|+\frac{z_{\alpha} \Omega^{2-\varepsilon}}{\Gamma(\alpha-1) \Gamma(3-\varepsilon)}\left[\mu_{1}+\mu_{2}\right]\left\|\left(x_{1}-x_{2}, y_{1}-y_{2}\right)\right\| \\
& +\frac{z_{\alpha} \Omega^{1-\varepsilon}}{\Gamma(\alpha-1) \Gamma(2-\varepsilon)}\left[\mu_{1}+\mu_{2}\right]\left\|\left(x_{1}-x_{2}, y_{1}-y_{2}\right)\right\|+\frac{z_{\alpha} \Omega^{1-\varepsilon}}{\Gamma(2-\varepsilon)} \sum_{j=1}^{m} \hat{I}_{1 j}\left\|\left(x_{1}-x_{2}, y_{1}-y_{2}\right)\right\| \\
& +\frac{z_{\alpha} \Omega^{2-\varepsilon}}{\Gamma(3-\varepsilon)} \sum_{j=1}^{m} \breve{I}_{1 j}\left\|\left(x_{1}-x_{2}, y_{1}-y_{2}\right)\right\|+\frac{z_{\alpha}}{\Gamma(\alpha-1)}\left[\mu_{1}+\mu_{2}\right]\left\|\left(x_{1}-x_{2}, y_{1}-y_{2}\right)\right\| \\
& +\frac{z_{\alpha}}{\Gamma(\alpha-1)}\left[\mu_{1}+\mu_{2}\right]\left\|\left(x_{1}-x_{2}, y_{1}-y_{2}\right)\right\|+\frac{z_{\alpha} \Omega^{1-\varepsilon}}{\Gamma(2-\varepsilon)} \sum_{j=1}^{m} \check{I}_{1 j}\left\|\left(x_{1}-x_{2}, y_{1}-y_{2}\right)\right\| \\
& +z_{\alpha} \sum_{j=1}^{m} \check{I}_{1 j}\left\|\left(x_{1}-x_{2}, y_{1}-y_{2}\right)\right\|+\frac{1}{2} \sum_{j=1}^{m} \check{I}_{1 j}\left\|\left(x_{1}-x_{2}, y_{1}-y_{2}\right)\right\| \\
& \leqslant\left[( \mu _ { 1 } + \mu _ { 2 } ) \left(\frac{2}{\Gamma(\alpha+1)}+\frac{1}{\Gamma(\alpha)}+\frac{1}{2 \Gamma(\alpha-1)}+\frac{z_{\alpha}}{\Gamma(\alpha-\varepsilon+1)}+\frac{z_{\alpha} \Omega^{1-\varepsilon}}{\Gamma(\alpha) \Gamma(2-\varepsilon)}+\frac{z_{\alpha} \Omega^{2-\varepsilon}}{\Gamma(\alpha-1) \Gamma(3-\varepsilon)}\right.\right. \\
& \left.+\frac{z_{\alpha} \Omega^{1-\varepsilon}}{\Gamma(\alpha-1) \Gamma(2-\varepsilon)}+\frac{2 z_{\alpha}}{\Gamma(\alpha-1)}\right)+\sum_{j=1}^{m} I_{1 j}+\sum_{j=1}^{m} \hat{I}_{1 j}+\frac{z_{\alpha} \Omega^{1-\varepsilon}}{\Gamma(2-\varepsilon)} \sum_{j=1}^{m} \hat{\mathrm{I}}_{1 j}+\frac{z_{\alpha} \Omega^{2-\varepsilon}}{\Gamma(3-\varepsilon)} \sum_{j=1}^{m} \check{I}_{1 j} \\
& \left.+\frac{z_{\alpha} \Omega^{1-\varepsilon}}{\Gamma(2-\varepsilon)} \sum_{j=1}^{m} \breve{I}_{1 j}+z_{\alpha} \sum_{j=1}^{m} \breve{I}_{1 j}+\frac{1}{2} \sum_{j=1}^{m} \check{I}_{1 j}\right]\left\|\left(x_{1}-x_{2}, y_{1}-y_{2}\right)\right\| \text {. }
\end{aligned}
$$

In the same fashion, we can obtain

$$
\begin{array}{ll} 
& \left|\mathbf{W}_{2}\left(x_{1}, y_{1}\right)(t)-\mathbf{W}_{2}\left(x_{2}, y_{2}\right)(t)\right| \\
\leqslant & z_{\beta}\left[( \mu _ { 1 } ^ { \prime } + \mu _ { 2 } ^ { \prime } ) \left(\frac{1}{z_{\beta} \Gamma(\beta+1)}+\frac{1}{\Gamma(\beta-\rho+1)}+\frac{\Phi^{1-\rho}}{\Gamma(\beta) \Gamma(2-\rho)}+\frac{\Phi^{2-\rho}}{\Gamma(\beta-1) \Gamma(3-\rho)}+\frac{\Phi^{1-\rho}}{\Gamma(\beta-1) \Gamma(2-\rho)}\right.\right. \\
& \left.\left.+\frac{2}{\Gamma(\beta-1)}\right)+\frac{\Phi^{1-\rho}}{\Gamma(2-\rho)} \sum_{j=1}^{m} \hat{I}_{2 j}+\frac{\Phi^{2-\rho}}{\Gamma(3-\rho)} \sum_{j=1}^{m} \check{I}_{2 j}+\frac{\Phi^{1-\rho}}{\Gamma(2-\rho)} \sum_{j=1}^{m} \breve{I}_{2 j}+\sum_{j=1}^{m} \breve{I}_{2 j}\right] \\
& \times\left\|\left(x_{1}-x_{2}, y_{1}-y_{2}\right)\right\|, t \in\left[0, t_{1}\right],
\end{array}
$$


and

$$
\begin{aligned}
& \left|\mathbf{W}_{2}\left(x_{1}, y_{1}\right)(t)-\mathbf{W}_{2}\left(x_{2}, y_{2}\right)(t)\right| \\
\leqslant & {\left[( \mu _ { 1 } ^ { \prime } + \mu _ { 2 } ^ { \prime } ) \left(\frac{2}{\Gamma(\beta+1)}+\frac{1}{\Gamma(\beta)}+\frac{1}{2 \Gamma(\beta-1)}+\frac{z_{\beta}}{\Gamma(\beta-\rho+1)}+\frac{z_{\beta} \Phi^{1-\rho}}{\Gamma(\beta) \Gamma(2-\rho)}+\frac{z_{\beta} \Phi^{2-\rho}}{\Gamma(\beta-1) \Gamma(3-\rho)}\right.\right.} \\
& \left.+\frac{z_{\beta} \Phi^{1-\rho}}{\Gamma(\beta-1) \Gamma(2-\rho)}+\frac{2 z_{\beta}}{\Gamma(\beta-1)}\right)+\sum_{j=1}^{m} I_{2 j}+\sum_{j=1}^{m} \hat{I}_{2 j}+\frac{z_{\beta} \Phi^{1-\rho}}{\Gamma(2-\rho)} \sum_{j=1}^{m} \hat{\mathrm{I}}_{2 j}+\frac{z_{\beta} \Phi^{2-\rho}}{\Gamma(3-\rho)} \sum_{j=1}^{m} \check{I}_{2 j} \\
& \left.+\frac{z_{\beta} \Phi^{1-\rho}}{\Gamma(2-\rho)} \sum_{j=1}^{m} \check{I}_{2 j}+z_{\beta} \sum_{j=1}^{m} \check{\mathrm{I}}_{2 j}+\frac{1}{2} \sum_{j=1}^{m} \check{\mathrm{I}}_{2 j}\right]\left\|\left(x_{1}-x_{2}, y_{1}-y_{2}\right)\right\|, t \in\left(t_{m}, t_{m+1}\right] .
\end{aligned}
$$

Thus, from (18)-(22) and $\left(\mathbf{M}_{3}\right)$, we infer that $\mathbf{W}$ is a contraction mapping. According to Lemma 3 , $\mathbf{W}$ has a fixed point $\left(x^{*}(t), y^{*}(t)\right) \in Y^{\prime}$, which is unique. Therefore, problem (1) has a unique solution $\left(x^{*}(t), y^{*}(t)\right)$.

Theorem 3. Let $\left(\mathbf{M}_{1}\right)-\left(\mathbf{M}_{2}\right),\left(\mathbf{M}_{4}\right)$ and for all $\mathrm{t} \in \mathrm{J}$ such that $\mathrm{h}(\mathrm{t}, 0,0)=w(\mathrm{t}, 0,0)=0, \mathrm{M}_{\mathrm{ik}}=\mathrm{N}_{\mathrm{ik}}=$ $\mathrm{O}_{i \mathrm{k}}=0,(\mathrm{i}=1,2 ; \mathrm{k}=1,2, \ldots, \mathrm{n})$ hold. Then, (1) has at least one solution $\left(\mathrm{x}^{*}(\mathrm{t}), \mathrm{y}^{*}(\mathrm{t})\right)$.

Proof. For the sake of simplicity, let us denote

$$
\begin{aligned}
& \varpi=\left[( \mu _ { 1 } + \mu _ { 2 } ) \left(\frac{3}{\Gamma(\alpha+1)}+\frac{1}{\Gamma(\alpha)}+\frac{1}{2 \Gamma(\alpha-1)}+\frac{z_{\alpha}}{\Gamma(\alpha-\varepsilon+1)}+\frac{z_{\alpha} \Omega^{1-\varepsilon}}{\Gamma(\alpha) \Gamma(2-\varepsilon)}+\frac{z_{\alpha} \Omega^{2-\varepsilon}}{\Gamma(\alpha-1) \Gamma(3-\varepsilon)}\right.\right. \\
& \left.+\frac{z_{\alpha} \Omega^{1-\varepsilon}}{\Gamma(\alpha-1) \Gamma(2-\varepsilon)}+\frac{2 z_{\alpha}}{\Gamma(\alpha-1)}\right)+\sum_{j=1}^{m} \mathrm{I}_{1 j}+\sum_{j=1}^{m} \hat{\mathrm{I}}_{1 j}+\frac{z_{\alpha} \Omega^{1-\varepsilon}}{\Gamma(2-\varepsilon)} \sum_{j=1}^{\mathrm{m}} \hat{\mathrm{I}}_{1 j}+\frac{z_{\alpha} \Omega^{2-\varepsilon}}{\Gamma(3-\varepsilon)} \sum_{j=1}^{\mathrm{m}} \breve{\mathrm{I}}_{1 j} \\
& \left.+\frac{z_{\alpha} \Omega^{1-\varepsilon}}{\Gamma(2-\varepsilon)} \sum_{j=1}^{m} \breve{I}_{1 j}+z_{\alpha} \sum_{j=1}^{m} \breve{I}_{1 j}+\frac{1}{2} \sum_{j=1}^{m} \breve{I}_{1 j}\right] \text {, } \\
& \xi=\left[( \mu _ { 1 } ^ { \prime } + \mu _ { 2 } ^ { \prime } ) \left(\frac{3}{\Gamma(\beta+1)}+\frac{1}{\Gamma(\beta)}+\frac{1}{2 \Gamma(\beta-1)}+\frac{z_{\beta}}{\Gamma(\beta-\rho+1)}+\frac{z_{\beta} \Phi^{1-\rho}}{\Gamma(\beta) \Gamma(2-\rho)}+\frac{z_{\beta} \Phi^{2-\rho}}{\Gamma(\beta-1) \Gamma(3-\rho)}\right.\right. \\
& \left.+\frac{z_{\beta} \Phi^{1-\rho}}{\Gamma(\beta-1) \Gamma(2-\rho)}+\frac{2 z_{\beta}}{\Gamma(\beta-1)}\right)+\sum_{j=1}^{m} I_{2 j}+\sum_{j=1}^{m} \hat{\mathrm{I}}_{2 j}+\frac{z_{\beta} \Phi^{1-\rho}}{\Gamma(2-\rho)} \sum_{j=1}^{m} \hat{\mathrm{I}}_{2 j}+\frac{z_{\beta} \Phi^{2-\rho}}{\Gamma(3-\rho)} \sum_{j=1}^{m} \check{\mathrm{I}}_{2 j} \\
& \left.+\frac{z_{\beta} \Phi^{1-\rho}}{\Gamma(2-\rho)} \sum_{j=1}^{m} \breve{I}_{2 j}+z_{\beta} \sum_{j=1}^{m} \breve{I}_{2 j}+\frac{1}{2} \sum_{j=1}^{m} \breve{I}_{2 j}\right] \text {, }
\end{aligned}
$$

and $\mathcal{R}_{\mathcal{v}}=\max \left\{\left(\frac{1}{\boldsymbol{\omega}}+1, \frac{1}{\xi}+1\right)\right\}$. Define the operator $\mathbf{W}$, as in (17), and a closed ball of Banach space $\mathbf{Y}^{\prime}$ as follows:

$$
v=\left\{(x, y) \in Y^{\prime}:\|(x, y)\| \leqslant \mathcal{R}_{v}\right\} .
$$

Similar to (18)-(22), we easily show that $\mathbf{W}(v) \subset v$ by applying $\left(\mathbf{M}_{4}\right) . \mathbf{W}(v) \subset v$ indicates that $\mathbf{W}(v)$ is uniformly bounded in $Y^{\prime}$. The continuity of the operator $\mathbf{W}$ is follows from the continuity of $h, w, M_{i m}, N_{i m}$ and $O_{i m}$. Now, we need to prove that $\mathbf{W}: v \rightarrow v$ is equicontinuous. Let $(x, y) \in v$ and $\ell_{1}, \ell_{2} \in[0,1]$ with $\ell_{1}<\ell_{2}$. When $0 \leqslant \ell_{1}<\ell_{2} \leqslant t_{1}$, similar to Equation (18), we have

$$
\begin{aligned}
& \left|\mathbf{W}_{1}(x, y)\left(\ell_{2}\right)-\mathbf{W}_{1}(x, y)\left(\ell_{1}\right)\right| \\
& =\mid \frac{1}{\Gamma(\alpha)} \int_{0}^{\ell_{1}}\left[\left(\ell_{2}-s\right)^{\alpha-1}-\left(\ell_{1}-s\right)^{\alpha-1}\right] h\left(s^{c}{ }^{c} D^{a} x(s),{ }^{c} D^{b} y(s)\right) d s+\frac{1}{\Gamma(\alpha)} \int_{\ell_{1}}^{\ell_{2}}\left(\ell_{2}-s\right)^{\alpha-1} \\
& \times h\left(s,{ }^{c} D^{a} x(s),{ }^{c} D^{b} y(s)\right) d s-e^{*}\left(\ell_{2}-\ell_{1}\right)^{2},
\end{aligned}
$$




$$
\begin{aligned}
& \leqslant \frac{1}{\Gamma(\alpha)} \int_{0}^{\ell_{1}}\left[\left(\ell_{2}-s\right)^{\alpha-1}-\left(\ell_{1}-s\right)^{\alpha-1}\right]\left|h\left(s,{ }^{c} D^{a} x(s),{ }^{c} D^{b} y(s)\right)\right| d s+\frac{1}{\Gamma(\alpha)} \int_{\ell_{1}}^{\ell_{2}}\left(\ell_{2}-s\right)^{\alpha-1} \\
& \times\left|h\left(s,{ }^{c} D^{a} x(s),{ }^{c} D^{b} y(s)\right)\right| d s+\left|e^{*}\right|\left(\ell_{2}-\ell_{1}\right)^{2}, \\
& \leqslant \frac{\mu_{1}+\mu_{2}}{\Gamma(\alpha)}\|(x, y)\| \int_{0}^{\ell_{1}}\left[\left(\ell_{2}-s\right)^{\alpha-1}-\left(\ell_{1}-s\right)^{\alpha-1}\right] \mathrm{d} s+\frac{\mu_{1}+\mu_{2}}{\Gamma(\alpha+1)}\|(x, y)\|\left(\ell_{2}-\ell_{1}\right)^{\alpha}+\left|e^{*}\right|\left(\ell_{2}-\ell_{1}\right)^{2} \text {, } \\
& \leqslant \frac{\mu_{1}+\mu_{2}}{\Gamma(\alpha+1)}\|(x, y)\|\left[\left(\ell_{2}-\ell_{1}\right)^{\alpha}-\left(\ell_{2}^{\alpha}-\ell_{1}^{\alpha}\right)\right]+\frac{\mu_{1}+\mu_{2}}{\Gamma(\alpha+1)}\|(x, y)\|\left(\ell_{2}-\ell_{1}\right)^{\alpha}+\left|e^{*}\right|\left(\ell_{2}-\ell_{1}\right)^{2} \text {, } \\
& \leqslant z_{\alpha}\left[( \mu _ { 1 } + \mu _ { 2 } ) \left(\frac{2}{z_{\alpha} \Gamma(\alpha+1)}+\frac{1}{\Gamma(\alpha-\varepsilon+1)}+\frac{\Omega^{1-\varepsilon}}{\Gamma(\alpha) \Gamma(2-\varepsilon)}+\frac{\Omega^{2-\varepsilon}}{\Gamma(\alpha-1) \Gamma(3-\varepsilon)}+\frac{\Omega^{1-\varepsilon}}{\Gamma(\alpha-1) \Gamma(2-\varepsilon)}\right.\right. \\
& \left.\left.+\frac{2}{\Gamma(\alpha-1)}\right)+\frac{\Omega^{1-\varepsilon}}{\Gamma(2-\varepsilon)} \sum_{j=1}^{m} \hat{\mathrm{I}}_{1 j}+\frac{\Omega^{2-\varepsilon}}{\Gamma(3-\varepsilon)} \sum_{j=1}^{m} \check{\mathrm{I}}_{1 j}+\frac{\Omega^{1-\varepsilon}}{\Gamma(2-\varepsilon)} \sum_{j=1}^{m} \check{\mathrm{I}}_{1 j}+\sum_{j=1}^{m} \check{\mathrm{I}}_{1 j}\right]\|x, y\|\left(\ell_{2}-\ell_{1}\right)^{2}, \\
& \leqslant \varpi \mathcal{R}_{v}\left(\ell_{2}-\ell_{1}\right)^{2} \text {. }
\end{aligned}
$$

In the same fashion, we obtain

$$
\left|\mathbf{W}_{2}(x, y)\left(\ell_{2}\right)-\mathbf{W}_{2}(x, y)\left(\ell_{1}\right)\right| \leqslant \xi \mathcal{R}_{v}\left(\ell_{2}-\ell_{1}\right)^{2}
$$

In addition, we obtain the same result when $t_{m}<\ell_{1}<\ell_{2} \leqslant t_{m+1}, 1 \leqslant m \leqslant n$, similar to (20)

$$
\left|\mathbf{W}_{1}(x, y)\left(\ell_{2}\right)-\mathbf{W}_{1}(x, y)\left(\ell_{1}\right)\right| \leqslant \varpi \mathcal{R}_{v}\left(\ell_{2}-\ell_{1}\right)^{2}
$$

and

$$
\left|\mathbf{W}_{2}(x, y)\left(\ell_{2}\right)-\mathbf{W}_{2}(x, y)\left(\ell_{1}\right)\right| \leqslant \xi \mathcal{R}_{v}\left(\ell_{2}-\ell_{1}\right)^{2} .
$$

Thus, it follows from (24)-(27) that, for any $\epsilon>0$, there exists a positive constant $\sigma=\frac{\epsilon}{\mathcal{R}_{v}} \min \left\{\frac{1}{\omega}, \frac{1}{\xi}\right\}$ independent of $\ell_{1}, \ell_{2}$ and $(x, y)$ such that $\left\|\mathbf{W}(x, y)\left(\ell_{2}\right)-\mathbf{W}(x, y)\left(\ell_{1}\right)\right\|<\epsilon$, whenever $\left|\ell_{2}-\ell_{1}\right| \leqslant \sigma$. Thereby, $\mathbf{W}: Y^{\prime} \rightarrow Y^{\prime}$ is equicontinuous. By the Arzela-Ascoli theorem, we know that $\mathbf{W}: Y^{\prime} \rightarrow Y^{\prime}$ is completely continuous. In view of Theorem $1, \mathbf{W}$ has a unique fixed point $\left(x^{*}(t), y^{*}(t)\right) \in \bar{v}$, which is a solution of system (1).

\section{Ulam-Hyers Stability}

In this section, we are interested in Ulam-Hyers stability and its types for the solution of (1).

Definition 4. [61] Problem (1) is Ulam-Hyers stable if there exists a constant $\mathbf{K}_{\alpha, \beta}=\left(\mathbf{K}_{\alpha}, \mathbf{K}_{\beta}\right)>0$ such that, for any $\epsilon=\left(\epsilon_{\alpha}, \epsilon_{\beta}\right)>0$, and $m=1,2, \ldots, n$, there exists a solution $(x, y) \in Y^{\prime}$ of:

$$
\left\{\begin{array}{l}
\left|{ }^{c} D^{\alpha} x(t)-h\left(t^{c}{ }^{c} D^{a} x(t),{ }^{c} D^{b} y(t)\right)\right| \leqslant \epsilon_{\alpha}, \\
\left|\Delta x\left(t_{m}\right)-M_{1 m}\left(x\left(t_{m}\right)\right)\right| \leqslant \epsilon_{\alpha}, \\
\left|\Delta x^{\prime}\left(t_{m}\right)-N_{1 m}\left(x\left(t_{m}\right)\right)\right| \leqslant \epsilon_{\alpha}, \\
\left|\Delta x^{\prime \prime}\left(t_{m}\right)-O_{1 m}\left(x\left(t_{m}\right)\right)\right| \leqslant \epsilon_{\alpha}, \\
\left|{ }^{c} D^{\beta} y(t)-w\left(t^{c}{ }^{c} D^{a} x(t),{ }^{c} D^{b} y(t)\right)\right| \leqslant \epsilon_{\beta}, \\
\left|\Delta y\left(t_{m}\right)-M_{2 m}\left(y\left(t_{m}\right)\right)\right| \leqslant \epsilon_{\beta}, \\
\left|\Delta y^{\prime}\left(t_{m}\right)-N_{2 m}\left(y\left(t_{m}\right)\right)\right| \leqslant \epsilon_{\beta}, \\
\left|\Delta y^{\prime \prime}\left(t_{m}\right)-O_{2 m}\left(y\left(t_{m}\right)\right)\right| \leqslant \epsilon_{\beta},
\end{array}\right.
$$

corresponding to a solution $(\zeta, \chi) \in Y^{\prime}$ of (1) such that

$$
|(x, y)(t)-(\zeta, x)(t)| \leqslant \mathbf{K}_{\alpha, \beta} \epsilon .
$$


Definition 5. [61] Problem (1) is generalized Ulam-Hyers stable if there exists a function $\Theta_{\alpha, \beta} \in \mathbf{C}\left(\mathbb{R}_{+}, \mathbb{R}_{+}\right)$, $\Theta_{\alpha, \beta}(0)=0$ for each $\epsilon>0$, such that for every solution $(x, y) \in Y^{\prime}$ of the inequality (28). there is a solution $(\zeta, \chi) \in Y^{\prime}$ of (1) such that

$$
|(x, y)(t)-(\zeta, \chi)(t)| \leqslant \Theta_{\alpha, \beta}(\epsilon) .
$$

Definition 6. [61] Problem (1) is Ulam-Hyers-Rassias stable with respect to $\left(\Psi_{\alpha, \beta}, \varphi_{\alpha, \beta}\right)$, where $\Psi_{\alpha, \beta}=$ $\left(\Psi_{\alpha}, \Psi_{\beta}\right) \in \mathrm{C}(J, \mathbb{R})$ and $\varphi_{\alpha, \beta}=\left(\varphi_{\alpha}, \varphi_{\beta}\right) \in \mathrm{C}(J, \mathbb{R})$, if, for every $\epsilon=\left(\epsilon_{\alpha}, \epsilon_{\beta}\right)>0$, there exists a real number $\mathbf{K}_{\Psi, \varphi}>0$, such that for $\mathrm{m}=1,2, \ldots, \mathrm{n}$ and for a solution $(x, y) \in \mathrm{Y}^{\prime}$ of:

$$
\left\{\begin{array}{l}
\left|{ }^{\mathrm{c}} \mathbf{D}^{\alpha} x(\mathrm{t})-\mathrm{h}\left(\mathrm{t}^{\mathrm{c}} \mathbf{D}^{\mathrm{a}} x(\mathrm{t}),{ }^{\mathrm{c}} \mathbf{D}^{\mathrm{b}} \mathrm{y}(\mathrm{t})\right)\right| \leqslant \Psi_{\alpha}(\mathrm{t}), \\
\left|\Delta x\left(\mathrm{t}_{\mathrm{m}}\right)-\mathrm{M}_{1 m}\left(x\left(\mathrm{t}_{\mathrm{m}}\right)\right)\right| \leqslant \varphi_{\alpha}, \\
\left|\Delta x^{\prime}\left(\mathrm{t}_{\mathrm{m}}\right)-\mathrm{N}_{1 m}\left(x\left(\mathrm{t}_{\mathrm{m}}\right)\right)\right| \leqslant \varphi_{\alpha}, \\
\left|\Delta x^{\prime \prime}\left(\mathrm{t}_{\mathrm{m}}\right)-\mathrm{O}_{1 m}\left(x\left(\mathrm{t}_{\mathrm{m}}\right)\right)\right| \leqslant \varphi_{\alpha}, \\
\left|{ }^{\mathrm{c}} \mathbf{D}^{\beta} \mathrm{y}(\mathrm{t})-w\left(\mathrm{t}^{\mathrm{c}} \mathbf{D}^{\mathrm{a}} x(\mathrm{t}),{ }^{\mathrm{c}} \mathbf{D}^{\mathrm{b}} \mathrm{y}(\mathrm{t})\right)\right| \leqslant \Psi_{\beta}(\mathrm{t}), \\
\left|\Delta \mathrm{y}\left(\mathrm{t}_{\mathrm{m}}\right)-\mathrm{M}_{2 \mathrm{~m}}\left(\mathrm{y}\left(\mathrm{t}_{\mathrm{m}}\right)\right)\right| \leqslant \varphi_{\beta}, \\
\left|\Delta \mathrm{y}^{\prime}\left(\mathrm{t}_{\mathrm{m}}\right)-\mathrm{N}_{2 m}\left(\mathrm{y}\left(\mathrm{t}_{\mathrm{m}}\right)\right)\right| \leqslant \varphi_{\beta}, \\
\left|\Delta \mathrm{y}^{\prime \prime}\left(\mathrm{t}_{\mathrm{m}}\right)-\mathrm{O}_{2 m}\left(\mathrm{y}\left(\mathrm{t}_{\mathrm{m}}\right)\right)\right| \leqslant \varphi_{\beta},
\end{array}\right.
$$

there is a solution $(\zeta, \chi) \in Y^{\prime}$ of (1) such that

$$
|(x, y)(t)-(\zeta, x)(t)| \leqslant \mathbf{K}_{\Psi, \varphi}\left(\Psi_{\alpha, \beta}(t)+\varphi_{\alpha, \beta}\right) \epsilon .
$$

Definition 7. [61] Problem (1) is generalized Ulam-Hyers-Rassias stable with respect to $\left(\Psi_{\alpha, \beta}, \varphi_{\alpha, \beta}\right) \in$ $C(J, \mathbb{R})$, if there exists a real number $K_{\Psi, \varphi}>0$, such that for $m=1,2, \ldots, n$ and for every solution $(x, y) \in Y^{\prime}$ of the following:

$$
\left\{\begin{array}{l}
\left|{ }^{\mathrm{c}} \mathbf{D}^{\alpha} x(t)-h\left(t^{c}{ }^{c} D^{a} x(t),{ }^{c} D^{b} y(t)\right)\right| \leqslant \Psi_{\alpha}(t) \epsilon_{\alpha}, \\
\left|\Delta x\left(t_{m}\right)-M_{1 m}\left(x\left(t_{m}\right)\right)\right| \leqslant \varphi_{\alpha} \epsilon_{\alpha}, \\
\left|\Delta x^{\prime}\left(t_{m}\right)-N_{1 m}\left(x\left(t_{m}\right)\right)\right| \leqslant \varphi_{\alpha} \epsilon_{\alpha}, \\
\left|\Delta x^{\prime \prime}\left(t_{m}\right)-O_{1 m}\left(x\left(t_{m}\right)\right)\right| \leqslant \varphi_{\alpha} \epsilon_{\alpha}, \\
\left|{ }^{c} D^{\beta} y(t)-w\left(t^{c}{ }^{c} D^{a} x(t),^{c} D^{b} y(t)\right)\right| \leqslant \Psi_{\beta}(t) \epsilon_{\beta}, \\
\left|\Delta y\left(t_{m}\right)-M_{2 m}\left(y\left(t_{m}\right)\right)\right| \leqslant \varphi_{\beta} \epsilon_{\beta}, \\
\left|\Delta y^{\prime}\left(t_{m}\right)-N_{2 m}\left(y\left(t_{m}\right)\right)\right| \leqslant \varphi_{\beta} \epsilon_{\beta}, \\
\left|\Delta y^{\prime \prime}\left(t_{m}\right)-O_{2 m}\left(y\left(t_{m}\right)\right)\right| \leqslant \varphi_{\beta} \epsilon_{\beta},
\end{array}\right.
$$

there is a solution $(\zeta, \chi) \in Y^{\prime}$ of (1) such that

$$
|(x, y)(t)-(\zeta, \chi)(t)| \leqslant K_{\Psi, \varphi}\left(\Psi_{\alpha, \beta}(t)+\varphi_{\alpha, \beta}\right) .
$$

Remark 1. A function $(x, y) \in Y^{\prime}$ is a solution of the inequality (28), if and only if there exist functions $\digamma_{h}, \hat{\digamma}_{w} \in Y^{\prime}$ and a sequence $\digamma_{m}, \hat{\digamma}_{m}, \mathrm{~m}=1,2, \ldots, \mathrm{n}$ depending on $(\mathrm{x}, \mathrm{y})$, such that

- $\left|\digamma_{h}(\mathrm{t})\right| \leqslant \epsilon_{\alpha},\left|\hat{\digamma}_{w}(\mathrm{t})\right| \leqslant \epsilon_{\beta},\left|\digamma_{m}\right| \leqslant \epsilon_{\alpha},\left|\hat{\digamma}_{m}\right| \leqslant \epsilon_{\beta}, \mathrm{t} \in J_{m}, \mathrm{~m}=1, \ldots, \mathrm{n} ;$

- ${ }^{c} D^{\alpha} x(t)=-h\left(t,{ }^{c} D^{a} x(t),{ }^{c} D^{b} y(t)\right)+\digamma_{h}(t)$;

- $\left.\Delta x\right|_{t=t_{m}}=M_{1 m}\left(x\left(t_{m}\right)\right)+\digamma_{m}$;

- $\left.\Delta x\right|_{t=t_{m}}=N_{1 m}\left(x\left(t_{m}\right)\right)+\digamma_{m} ;$

- $\left.\Delta x\right|_{\mathrm{t}=\mathrm{t}_{\mathrm{m}}}=\mathrm{O}_{1 \mathrm{~m}}\left(\mathrm{x}\left(\mathrm{t}_{\mathrm{m}}\right)\right)+\digamma_{\mathrm{m}}$;

- ${ }^{c} D^{\beta} x(t)=-w\left(t,{ }^{c} D^{a} x(t),{ }^{c} D^{b} y(t)\right)+\hat{\digamma}_{w}(t)$;

- $\left.\Delta y\right|_{t=t_{m}}=M_{2 m}\left(y\left(t_{m}\right)\right)+\hat{\digamma}_{m} ;$

- $\left.\Delta y\right|_{t=t_{m}}=N_{2 m}\left(y\left(t_{m}\right)\right)+\hat{F}_{m}$;

- $\left.\Delta y\right|_{t=t_{m}}=O_{2 m}\left(y\left(t_{m}\right)\right)+\hat{\digamma}_{m}$. 
Remark 2. A function $(x, y) \in Y^{\prime}$ is a solution of the inequality (29), if and only if there exist functions $\digamma_{h}, \hat{\digamma}_{w} \in Y^{\prime}$ and a sequence $\digamma_{m}, \hat{\digamma}_{m}, \mathrm{~m}=1,2, \ldots, \mathrm{n}$ depending on $(\mathrm{x}, \mathrm{y})$, such that

- $\left|\digamma_{h}(t)\right| \leqslant \Psi_{\alpha},\left|\hat{\digamma}_{w}(t)\right| \leqslant \Psi_{\beta},\left|\digamma_{m}\right| \leqslant \varphi_{\alpha},\left|\digamma_{m}\right| \leqslant \varphi_{\beta}, t \in J_{m}, m=1, \ldots, n ;$

- ${ }^{c} D^{\alpha} x(t)=-h\left(t,{ }^{c} D^{a} x(t),{ }^{c} D^{b} y(t)\right)+\digamma_{h}(t) ;$

- $\left.\Delta x\right|_{t=t_{m}}=M_{1 m}\left(x\left(t_{m}\right)\right)+\digamma_{m} ;$

- $\left.\Delta x\right|_{t=t_{m}}=N_{1 m}\left(x\left(t_{m}\right)\right)+\digamma_{m} ;$

- $\left.\Delta x\right|_{t=t_{m}}=O_{1 m}\left(x\left(t_{m}\right)\right)+\digamma_{m} ;$

- ${ }^{c} D^{\beta} x(t)=-w\left(t^{c} D^{a} x(t),{ }^{c} D^{b} y(t)\right)+\hat{F}_{w}(t)$;

- $\left.\Delta y\right|_{t=t_{m}}=M_{2 m}\left(y\left(t_{m}\right)\right)+\hat{f}_{m} ;$

- $\left.\Delta y\right|_{t=t_{m}}=N_{2 m}\left(y\left(t_{m}\right)\right)+\hat{\digamma}_{m} ;$

- $\left.\Delta y\right|_{t=t_{m}}=O_{2 m}\left(y\left(t_{m}\right)\right)+\hat{f}_{m}$.

Similarly, one can easily state such a remark for the inequality (30).

Theorem 4. If the assumptions $\left(\mathbf{M}_{1}\right)-\left(\mathbf{M}_{2}\right)$ hold with

$$
\Lambda_{0}=1-\frac{\Lambda_{2}^{*} \Lambda_{4}^{*}}{\left(1-\Lambda_{1}^{*}\right)\left(1-\Lambda_{3}^{*}\right)}>0,
$$

then (1) is Ulam-Hyers and generalized Ulam-Hyers stable.

Proof. Let $(x, y) \in Y^{\prime}$ be any solution of the inequality (28) and let $(\zeta, x) \in Y^{\prime}$ be the unique solution of the following:

$$
\left\{\begin{array}{l}
{ }^{c} D^{\alpha} \zeta(t)+h\left(t^{c}{ }^{c} D^{a} \zeta(t),{ }^{c} D^{b} \chi(t)\right)=0, t \neq t_{m}, m=1,2, \ldots, n, \\
{ }^{c} D^{\beta} \chi(t)+w\left(t^{c} D^{a} \zeta(t),{ }^{c} D^{b} \chi(t)\right)=0, t \neq t_{m}, m=1,2, \ldots, n, \\
\left.\Delta \zeta\right|_{t=t_{m}}=M_{1 m}\left(\zeta\left(t_{m}\right)\right),\left.\Delta \zeta^{\prime}\right|_{t=t_{m}}=N_{1 m}\left(\zeta\left(t_{m}\right)\right),\left.\Delta \zeta^{\prime \prime}\right|_{t=t_{m}}=O_{1 m}\left(\zeta\left(t_{m}\right)\right), \\
\left.\Delta \chi\right|_{t=t_{m}}=M_{2 m}\left(\chi\left(t_{m}\right)\right),\left.\Delta \chi^{\prime}\right|_{t=t_{m}}=N_{2 m}\left(\chi\left(t_{m}\right)\right),\left.\Delta \chi^{\prime \prime}\right|_{t=t_{m}}=O_{2 m}\left(\chi\left(t_{m}\right)\right), \\
\zeta(0)=\zeta^{\prime}(0)=0,{ }^{c} D^{\varepsilon} \zeta(\Omega)=\zeta^{\prime \prime}(1), \\
\chi(0)=\chi^{\prime}(0)=0,{ }^{c} D^{\rho} \chi(\Phi)=\chi^{\prime \prime}(1) .
\end{array}\right.
$$

By Lemma 2.4, we have

$$
\left\{\begin{array}{l}
{ }^{c} D^{\alpha} x(t)+h\left(t^{c}{ }^{c} D^{a} x(t),{ }^{c} D^{b} y(t)\right)=\digamma_{h}(t), t \neq t_{m}, m=1,2 \ldots, n, \\
{ }^{c} D^{\beta} y(t)+w\left(t^{c} D^{a} x(t),{ }^{c} D^{b} y(t)\right)=\digamma_{w}(t), t \neq t_{m}, m=1,2 \ldots, n, \\
\left.\Delta x\right|_{t=t_{m}}=M_{1 m}\left(x\left(t_{m}\right)\right)+\digamma_{m},\left.\Delta x^{\prime}\right|_{t=t_{m}}=N_{1 m}\left(x\left(t_{m}\right)\right)+\digamma_{m},\left.\Delta x^{\prime \prime}\right|_{t=t_{m}}=O_{1 m}\left(x\left(t_{m}\right)\right)+\digamma_{m}, \\
\left.\Delta y\right|_{t=t_{m}}=M_{2 m}\left(y\left(t_{m}\right)\right)+\hat{\digamma}_{m},\left.\Delta y^{\prime}\right|_{t=t_{m}}=N_{2 m}\left(y\left(t_{m}\right)\right)+\hat{\digamma}_{m},\left.\Delta y^{\prime \prime}\right|_{t=t_{m}}=O_{2 m}\left(y\left(t_{m}\right)\right)+\hat{\digamma}_{m}, \\
x(0)=x^{\prime}(0)=0,{ }^{c} D^{\varepsilon} x(\Omega)=x^{\prime \prime}(1), \\
y(0)=y^{\prime}(0)=0,{ }^{c} D^{\rho} y(\Phi)=y^{\prime \prime}(1) .
\end{array}\right.
$$


Since $(x, y)$ is a solution of the inequality (28) and $t \in J$; hence, by Remark 1 , we obtain

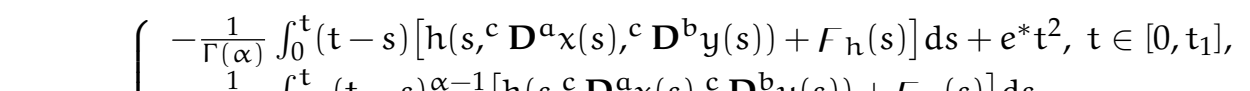

$$
\begin{aligned}
& -\frac{1}{\Gamma(\alpha)} \int_{t_{m}}^{t}(t-s)^{\alpha-1}\left[h\left(s,{ }^{c} D^{a} x(s),{ }^{c} D^{b} y(s)\right)+\digamma_{h}(s)\right] d s \\
& x(t)=\left\{\begin{array}{l}
-\frac{1}{\Gamma(\alpha)} \sum_{j=1}^{m} \int_{t_{j-1}}^{t_{j}}\left(t_{j}-s\right)^{\alpha-1}\left[h\left(s,{ }^{c} D^{a} x(s),{ }^{c} D^{b} y(s)\right)+\digamma_{h}(s)\right] d s \\
-\frac{1}{\Gamma(\alpha-1)} \sum_{j=1}^{m}\left(t-t_{j}\right) \int_{t_{j-1}}^{t_{j}}\left(t_{j}-s\right)^{\alpha-2}\left[h\left(s,{ }^{c} D^{a} x(s),{ }^{c} D^{b} y(s)\right)+\digamma_{h}(s)\right] d s
\end{array}\right. \\
& -\frac{1}{2 \Gamma(\alpha-2)} \sum_{j=1}^{m}\left(t-t_{j}\right)^{2} \int_{t_{j-1}}^{t_{j}}\left(t_{j}-s\right)^{\alpha-3}\left[h\left(s^{c}{ }^{c} D^{a} x(s){ }^{c}{ }^{c} D^{b} y(s)\right)+\digamma_{h}(s)\right] d s \\
& +\sum_{j=1}^{m}\left[M_{1 j}\left(x\left(t_{j}\right)\right)+\digamma_{m}\right]+\sum_{j=1}^{m}\left(t-t_{j}\right)\left[N_{1 j}\left(x\left(t_{j}\right)\right)+\digamma_{m}\right]+\sum_{j=1}^{m} \frac{\left(t-t_{j}\right)^{2}}{2} \\
& \times\left[\mathrm{O}_{1 j}\left(x\left(\mathrm{t}_{j}\right)\right)+\digamma_{\mathrm{m}}\right]+\mathrm{e}^{*} \mathrm{t}^{2}, \mathrm{t} \in\left(\mathrm{t}_{\mathrm{m}}, \mathrm{t}_{\mathrm{m}+1}\right], 1 \leqslant \mathrm{~m} \leqslant \mathrm{n} .
\end{aligned}
$$

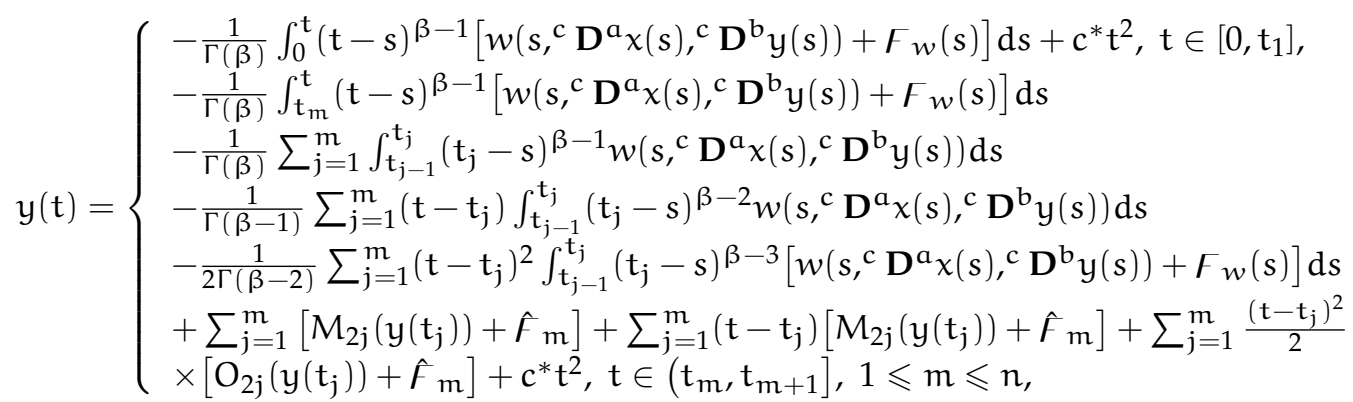

where

$$
\begin{aligned}
& e^{*}=z_{\alpha}\left[-\frac{1}{\Gamma(\alpha-\varepsilon)} \int_{t_{j}}^{\Omega}(\Omega-s)^{\alpha-\varepsilon-1}\left[h\left(s,{ }^{c} D^{a} x(s),{ }^{c} D^{b} y(s)\right)+\digamma_{h}(s)\right] d s\right. \\
& -\frac{\Omega^{1-\varepsilon}}{\Gamma(\alpha-1) \Gamma(2-\varepsilon)} \sum_{j=1}^{m} \int_{t_{j-1}}^{t_{j}}\left(t_{j}-s\right)^{\alpha-2}\left[h\left(s,{ }^{c} D^{a} x(s),{ }^{c} D^{b} y(s)\right)+\digamma_{h}(s)\right] d s \\
& -\frac{\Omega^{2-\varepsilon}}{\Gamma(\alpha-2) \Gamma(3-\varepsilon)} \sum_{j=1}^{m} \int_{t_{j-1}}^{t_{j}}\left(t_{j}-s\right)^{\alpha-3}\left[h\left(s,{ }^{c} D^{a} x(s),{ }^{c} D^{b} y(s)\right)+\digamma_{h}(s)\right] d s \\
& +\frac{\Omega^{1-\varepsilon}}{\Gamma(\alpha-2) \Gamma(2-\varepsilon)} \sum_{j=1}^{m} t_{j} \int_{t_{j-1}}^{t_{j}}\left(t_{j}-s\right)^{\alpha-3}\left[h\left(s,{ }^{c} D^{a} x(s),{ }^{c} D^{b} y(s)\right)+\digamma_{h}(s)\right] d s \\
& +\frac{\Omega^{1-\varepsilon}}{\Gamma(2-\varepsilon)} \sum_{j=1}^{m}\left[N_{1 j}\left(x\left(t_{j}\right)\right)+\digamma_{m}\right]+\frac{\Omega^{2-\varepsilon}}{\Gamma(3-\varepsilon)} \sum_{j=1}^{m}\left[O_{1 j}\left(x\left(t_{j}\right)\right)+\digamma_{m}\right]-\frac{\Omega^{1-\varepsilon}}{\Gamma(2-\varepsilon)} \sum_{j=1}^{m} t_{j} \\
& \times\left[\mathrm{O}_{1 j}\left(x\left(\mathrm{t}_{\mathrm{j}}\right)\right)+\digamma_{\mathrm{m}}\right]+\frac{1}{\Gamma(\alpha-2)} \int_{\mathrm{t}_{\mathrm{m}}}^{1}(1-\mathrm{s})^{\alpha-3}\left[\mathrm{~h}\left(\mathrm{~s}^{\mathrm{c}}{ }^{\mathrm{c}} \mathbf{D}^{\mathrm{a}} x(\mathrm{~s}),{ }^{\mathrm{c}} \mathbf{D}^{\mathrm{b}} \mathrm{y}(\mathrm{s})\right)+\digamma_{\mathrm{h}}(\mathrm{s})\right] \mathrm{d} \mathrm{s} \\
& \left.+\frac{1}{\Gamma(\alpha-2)} \sum_{j=1}^{m} \int_{t_{j-1}}^{t_{j}}\left(t_{j}-s\right)^{\alpha-3}\left[h\left(s,{ }^{c} D^{a} x(s),{ }^{c} D^{b} y(s)\right)+\digamma_{h}(s)\right] d s-\sum_{j=1}^{m}\left[O_{1 j}\left(x\left(t_{j}\right)\right)+\digamma_{m}\right]\right] \text {, } \\
& c^{*}=z_{\beta}\left[-\frac{1}{\Gamma(\beta-\rho)} \int_{\mathrm{t}_{j}}^{\Phi}(\Phi-s)^{\beta-\rho-1}\left[w\left(s^{c}{ }^{c} \mathbf{D}^{\mathrm{a}} x(s),{ }^{c} \mathbf{D}^{\mathrm{b}} \mathrm{y}(\mathrm{s})\right)+\digamma_{w}(\mathrm{~s})\right] \mathrm{d} s\right. \\
& -\frac{\Phi^{1-\rho}}{\Gamma(\beta-1) \Gamma(2-\rho)} \sum_{j=1}^{m} \int_{t_{j-1}}^{t_{j}}\left(t_{j}-s\right)^{\beta-2}\left[w\left(s,{ }^{c} D^{a} x(s),{ }^{c} D^{b} y(s)\right)+\digamma_{w}(s)\right] d s \\
& -\frac{\Phi^{2-\rho}}{\Gamma(\beta-2) \Gamma(3-\rho)} \sum_{j=1}^{m} \int_{t_{j-1}}^{t_{j}}\left(t_{j}-s\right)^{\beta-3}\left[w\left(s,{ }^{c} D^{a} x(s),{ }^{c} D^{b} y(s)\right)+\digamma_{w}(s)\right] d s \\
& +\frac{\Phi^{1-\rho}}{\Gamma(\beta-2) \Gamma(2-\rho)} \sum_{j=1}^{m} t_{j} \int_{t_{j-1}}^{t_{j}}\left(t_{j}-s\right)^{\beta-3}\left[w\left(s,{ }^{c} D^{a} x(s),{ }^{c} D^{b} y(s)\right)+\digamma w(s)\right] d s
\end{aligned}
$$




$$
\begin{aligned}
& +\frac{\Phi^{1-\rho}}{\Gamma(2-\rho)} \sum_{j=1}^{m}\left[\mathrm{~N}_{2 j}\left(y\left(t_{j}\right)\right)+\hat{\digamma}_{m}\right]+\frac{\Phi^{2-\rho}}{\Gamma(3-\rho)} \sum_{j=1}^{m}\left[\mathrm{O}_{2 j}\left(y\left(t_{j}\right)\right)+\hat{\digamma}_{m}\right]-\frac{\Phi^{1-\rho}}{\Gamma(2-\rho)} \sum_{j=1}^{m} t_{j} \\
& \times\left[O_{2 j}\left(y\left(t_{j}\right)\right)+\hat{\digamma}_{m}\right]+\frac{1}{\Gamma(\beta-2)} \int_{t_{m}}^{1}(1-s)^{\beta-3}\left[w\left(s^{c}{ }^{c} D^{a} x(s),{ }^{c} D^{b} y(s)\right)+\digamma_{w}(s)\right] d s \\
& \left.+\frac{1}{\Gamma(\beta-2)} \sum_{j=1}^{m} \int_{t_{j-1}}^{t_{j}}\left(t_{j}-s\right)^{\beta-3}\left[w\left(s,{ }^{c} D^{a} x(s),{ }^{c} D^{b} y(s)\right)+\digamma_{w}(s)\right] d s-\sum_{j=1}^{m}\left[O_{2 j}\left(y\left(t_{j}\right)\right)+\hat{\digamma}_{m}\right]\right] .
\end{aligned}
$$

For $\mathrm{t} \in\left[0, \mathrm{t}_{1}\right]$, we have

$$
\begin{aligned}
& x(t)=\frac{1}{\Gamma(\alpha)} \int_{0}^{t}(t-s)^{\alpha-1}\left[h\left(s,{ }^{c} D^{a} x_{1}(s),{ }^{c} D^{b} y_{1}(s)\right)+\digamma_{h}(s)\right] d s \\
& +\frac{z_{\alpha} t^{2}}{\Gamma(\alpha-\varepsilon)} \int_{t_{j}}^{\Omega}(\Omega-s)^{\alpha-\varepsilon-1}\left[h\left(s,{ }^{c} D^{a} x_{1}(s),{ }^{c} D^{b} y_{1}(s)\right)+\digamma_{h}(s)\right] d s \\
& +\frac{z_{\alpha} t^{2} \Omega^{1-\varepsilon}}{\Gamma(\alpha-1) \Gamma(2-\varepsilon)} \sum_{j=1}^{m} \int_{t_{j-1}}^{t_{j}}\left(t_{j}-s\right)^{\alpha-2}\left[h\left(s,{ }^{c} D^{a} x_{1}(s),{ }^{c} D^{b} y_{1}(s)\right)+\digamma_{h}(s)\right] d s \\
& +\frac{z_{\alpha} t^{2} \Omega^{2-\varepsilon}}{\Gamma(\alpha-2) \Gamma(3-\varepsilon)} \sum_{j=1}^{m} \int_{t_{j-1}}^{t_{j}}\left(t_{j}-s\right)^{\alpha-3}\left[h\left(s,{ }^{c} D^{a} x_{1}(s),{ }^{c} D^{b} y_{1}(s)\right)+\digamma_{h}(s)\right] d s \\
& +\frac{z_{\alpha} t^{2} \Omega^{1-\varepsilon}}{\Gamma(\alpha-2) \Gamma(2-\varepsilon)} \sum_{j=1}^{m} t_{j} \int_{t_{j-1}}^{t_{j}}\left(t_{j}-s\right)^{\alpha-3}\left[h\left(s,{ }^{c} D^{a} x_{1}(s),{ }^{c} D^{b} y_{1}(s)\right)+\digamma_{h}(s)\right] d s \\
& +\frac{z_{\alpha} t^{2} \Omega^{1-\varepsilon}}{\Gamma(2-\varepsilon)} \sum_{j=1}^{m}\left[N_{1 j}\left(x\left(t_{j}\right)\right)+\digamma_{m}\right]+\frac{z_{\alpha} t^{2} \Omega^{2-\varepsilon}}{\Gamma(3-\varepsilon)} \sum_{j=1}^{m}\left[O_{1 j}\left(x\left(t_{j}\right)\right)+\digamma_{m}\right] \\
& +\frac{z_{\alpha} t^{2}}{\Gamma(\alpha-2)} \int_{t_{m}}^{1}(1-s)^{\alpha-3}\left[h\left(s,{ }^{c} D^{a} x_{1}(s),{ }^{c} D^{b} y_{1}(s)\right)+\digamma_{h}(s)\right] d s \\
& +\frac{z_{\alpha} t^{2}}{\Gamma(\alpha-2)} \sum_{j=1}^{m} \int_{t_{j-1}}^{t_{j}}\left(t_{j}-s\right)^{\alpha-3}\left[h\left(s,{ }^{c} D^{a} x_{1}(s),{ }^{c} D^{b} y_{1}(s)\right)+\digamma_{h}(s)\right] d s \\
& +\frac{z_{\alpha} t^{2} \Omega^{1-\varepsilon}}{\Gamma(2-\varepsilon)} \sum_{j=1}^{m} t_{j}\left[O_{1 j}\left(x\left(t_{j}\right)\right)+\digamma_{m}\right]+z_{\alpha} t^{2} \sum_{j=1}^{m}\left[O_{1 j}\left(x\left(t_{j}\right)\right)+\digamma_{m}\right] .
\end{aligned}
$$

becomes

$$
\begin{aligned}
& \left|x(t)-s_{1}(t)\right| \\
\leqslant & \frac{1}{\Gamma(\alpha)} \int_{0}^{t}(t-s)^{\alpha-1}\left|\digamma_{h}(s)\right| d s+\frac{z_{\alpha}}{\Gamma(\alpha-\varepsilon)} \int_{t_{j}}^{\Omega}(\Omega-s)^{\alpha-\varepsilon-1}\left|\digamma_{h}(s)\right| d s \\
& +\frac{z_{\alpha} \Omega^{1-\varepsilon}}{\Gamma(\alpha-1) \Gamma(2-\varepsilon)} \sum_{j=1}^{m} \int_{t_{j-1}}^{t_{j}}\left(t_{j}-s\right)^{\alpha-2}\left|\digamma_{h}(s)\right| d s+\frac{z_{\alpha} \Omega^{2-\varepsilon}}{\Gamma(\alpha-2) \Gamma(3-\varepsilon)} \sum_{j=1}^{m} \int_{t_{j-1}}^{t_{j}}\left(t_{j}-s\right)^{\alpha-3}\left|\digamma_{h}(s)\right| d s \\
& +\frac{z_{\alpha} \Omega^{1-\varepsilon}}{\Gamma(\alpha-2) \Gamma(2-\varepsilon)} \sum_{j=1}^{m}\left|t_{j}\right| \int_{t_{j-1}}^{t_{j}}\left(t_{j}-s\right)^{\alpha-3}\left|\digamma_{h}(s)\right| d s+\frac{z_{\alpha} \Omega^{1-\varepsilon}}{\Gamma(2-\varepsilon)} \sum_{j=1}^{m}\left|\digamma_{m}\right|+\frac{z_{\alpha} \Omega^{2-\varepsilon}}{\Gamma(3-\varepsilon)} \sum_{j=1}^{m}\left|\digamma_{m}\right| \\
& +\frac{z_{\alpha}}{\Gamma(\alpha-2)} \int_{t_{m}}^{1}(1-s)^{\alpha-3}\left|\digamma_{h}(s)\right| d s+\frac{z_{\alpha}}{\Gamma(\alpha-2)} \sum_{j=1}^{m} \int_{t_{j-1}}^{t_{j}}\left(t_{j}-s\right)^{\alpha-3}\left|\digamma_{h}(s)\right| d s \\
& +\frac{z_{\alpha} \Omega^{1-\varepsilon}}{\Gamma(2-\varepsilon)} \sum_{j=1}^{m}\left|t_{j}\right|\left|\digamma_{m}\right|+z_{\alpha} \sum_{j=1}^{m}\left|\digamma_{m}\right| .
\end{aligned}
$$


By utilizing Remark 1, we get

$$
\begin{aligned}
& \left|x(t)-s_{1}(t)\right| \\
\leqslant & {\left[\frac{1}{\Gamma(\alpha+1)}+\frac{z_{\alpha}}{\Gamma(\alpha-\varepsilon+1)}+\frac{z_{\alpha} \Omega^{1-\varepsilon}}{\Gamma(\alpha) \Gamma(2-\varepsilon)}+\frac{z_{\alpha} \Omega^{2-\varepsilon}}{\Gamma(\alpha-1) \Gamma(3-\varepsilon)}+\frac{z_{\alpha} \Omega^{1-\varepsilon}}{\Gamma(\alpha-1) \Gamma(2-\varepsilon)}+\frac{2 z_{\alpha} \Omega^{1-\varepsilon}}{\Gamma(2-\varepsilon)}\right.} \\
& \left.+\frac{z_{\alpha} \Omega^{2-\varepsilon}}{\Gamma(3-\varepsilon)}+\frac{2 z_{\alpha}}{\Gamma(\alpha-1)}+z_{\alpha}\right] \epsilon_{\alpha} .
\end{aligned}
$$

Let

$$
\begin{aligned}
Q_{1} & =\frac{1}{\Gamma(\alpha+1)}+\frac{z_{\alpha}}{\Gamma(\alpha-\varepsilon+1)}+\frac{z_{\alpha} \Omega^{1-\varepsilon}}{\Gamma(\alpha) \Gamma(2-\varepsilon)}+\frac{z_{\alpha} \Omega^{2-\varepsilon}}{\Gamma(\alpha-1) \Gamma(3-\varepsilon)}+\frac{z_{\alpha} \Omega^{1-\varepsilon}}{\Gamma(\alpha-1) \Gamma(2-\varepsilon)}+\frac{2 z_{\alpha} \Omega^{1-\varepsilon}}{\Gamma(2-\varepsilon)} \\
& +\frac{z_{\alpha} \Omega^{2-\varepsilon}}{\Gamma(3-\varepsilon)}+\frac{2 z_{\alpha}}{\Gamma(\alpha-1)}+z_{\alpha} .
\end{aligned}
$$

Thus, (35) becomes

$$
\left|x(t)-s_{1}(t)\right| \leqslant Q_{1} \epsilon_{\alpha}
$$

Let

$$
|x(t)-\zeta(t)|=\left|x(t)-s_{1}(t)+s_{1}(t)-\zeta(t)\right| \leqslant\left|x(t)-s_{1}(t)\right|+\left|s_{1}(t)-\zeta(t)\right| .
$$

Using (36) in (37), we have

$$
\begin{aligned}
& |x(t)-\zeta(t)| \\
& \leqslant Q_{1} \epsilon_{\alpha}+\frac{1}{\Gamma(\alpha)} \int_{0}^{\mathrm{t}}(\mathrm{t}-\mathrm{s})^{\alpha-1}\left|\mathrm{~h}\left(\mathrm{~s}^{,}{ }^{\mathrm{c}} \mathbf{D}^{\mathrm{a}} \chi(\mathrm{s}),{ }^{\mathrm{c}} \mathbf{D}^{\mathrm{b}} \mathbf{y}(\mathrm{s})\right)-\mathrm{h}\left(\mathrm{s}^{,}{ }^{\mathrm{c}} \mathbf{D}^{\mathrm{a}} \zeta(\mathrm{s}),{ }^{\mathrm{c}} \mathbf{D}^{\mathrm{b}} \chi(\mathrm{s})\right)\right| \mathrm{d} s \\
& +\frac{z_{\alpha} t^{2}}{\Gamma(\alpha-\varepsilon)} \int_{t_{j}}^{\Omega}(\Omega-s)^{\alpha-\varepsilon-1}\left|h\left(s,{ }^{c} D^{a} x(s),{ }^{c} D^{b} y(s)\right)-h\left(s,{ }^{c} D^{a} \zeta(s),{ }^{c} D^{b} \chi(s)\right)\right| d s \\
& +\frac{z_{\alpha} t^{2} \Omega^{1-\varepsilon}}{\Gamma(\alpha-1) \Gamma(2-\varepsilon)} \sum_{j=1}^{m} \int_{t_{j-1}}^{t_{j}}\left(t_{j}-s\right)^{\alpha-2}\left|h\left(s,{ }^{c} D^{a} \chi(s),{ }^{c} D^{b} y(s)\right)-h\left(s,{ }^{c} D^{a} \zeta(s),{ }^{c} D^{b} \chi(s)\right)\right| d s \\
& +\frac{z_{\alpha} t^{2} \Omega^{2-\varepsilon}}{\Gamma(\alpha-2) \Gamma(3-\varepsilon)} \sum_{j=1}^{m} \int_{t_{j-1}}^{t_{j}}\left(t_{j}-s\right)^{\alpha-3}\left|h\left(s,{ }^{c} D^{a} x(s),{ }^{c} D^{b} y(s)\right)-h\left(s,{ }^{c} D^{a} \zeta(s),{ }^{c} D^{b} \chi(s)\right)\right| d s \\
& +\frac{z_{\alpha} t^{2} \Omega^{1-\varepsilon}}{\Gamma(\alpha-2) \Gamma(2-\varepsilon)} \sum_{j=1}^{m} t_{j} \int_{t_{j-1}}^{t_{j}}\left(t_{j}-s\right)^{\alpha-3}\left|h\left(s,{ }^{c} D^{a} \chi(s),{ }^{c} D^{b} y(s)\right)-h\left(s,{ }^{c} D^{a} \zeta(s),{ }^{c} D^{b} \chi(s)\right)\right| d s \\
& +\frac{z_{\alpha} t^{2} \Omega^{1-\varepsilon}}{\Gamma(2-\varepsilon)} \sum_{j=1}^{m}\left|N_{1 j}\left(x\left(t_{j}\right)\right)-N_{1 j}\left(\zeta\left(t_{j}\right)\right)\right|+\frac{z_{\alpha} t^{2} \Omega^{2-\varepsilon}}{\Gamma(3-\varepsilon)} \sum_{j=1}^{m}\left|O_{1 j}\left(x\left(t_{j}\right)\right)-O_{1 j}\left(\zeta\left(t_{j}\right)\right)\right| \\
& +\frac{z_{\alpha} \mathrm{t}^{2}}{\Gamma(\alpha-2)} \int_{\mathrm{t}_{\mathrm{m}}}^{1}(1-\mathrm{s})^{\alpha-3}\left|\mathrm{~h}\left(\mathrm{~s}^{\mathrm{c}}, \mathbf{D}^{\mathrm{a}} x(\mathrm{~s}),{ }_{,}^{\mathrm{c}} \mathbf{D}^{\mathrm{b}} \mathrm{y}(\mathrm{s})\right)-\mathrm{h}\left(\mathrm{s}^{\mathrm{c}}, \mathbf{D}^{\mathrm{a}} \zeta(\mathrm{s}),{ }^{\mathrm{c}} \mathbf{D}^{\mathrm{b}} \chi(\mathrm{s})\right)\right| \mathrm{d} \mathrm{s} \\
& +\frac{z_{\alpha} t^{2}}{\Gamma(\alpha-2)} \sum_{j=1}^{m} \int_{t_{j-1}}^{t_{j}}\left(t_{j}-s\right)^{\alpha-3}\left|h\left(s,{ }^{c} D^{a} \chi(s),{ }^{c} D^{b} y(s)\right)-h\left(s,{ }^{c} D^{a} \zeta(s),{ }^{c} D^{b} \chi(s)\right)\right| d s \\
& +\frac{z_{\alpha} t^{2} \Omega^{1-\varepsilon}}{\Gamma(2-\varepsilon)} \sum_{j=1}^{m}\left|t_{j}\right|\left|O_{1 j}\left(x\left(t_{j}\right)\right)-O_{1 j}\left(\zeta\left(t_{j}\right)\right)\right|+z_{\alpha} t^{2} \sum_{j=1}^{m}\left|O_{1 j}\left(x\left(t_{j}\right)\right)-O_{1 j}\left(\zeta\left(t_{j}\right)\right)\right| .
\end{aligned}
$$


Utilizing $\left(\mathbf{M}_{1}\right)$ and $\left(\mathbf{M}_{2}\right)$, we get

$$
\begin{aligned}
\leqslant & Q_{1} \epsilon_{\alpha}+\frac{1}{\Gamma(\alpha+1)}\left[\mu_{1}\|x-\zeta\|_{0}+\mu_{2}\|y-\chi\|_{0}\right]+\frac{z_{\alpha}}{\Gamma(\alpha-\varepsilon+1)}\left[\mu_{1}\|x-\zeta\|_{0}+\mu_{2}\|y-\chi\|_{0}\right] \\
& +\frac{z_{\alpha} \Omega^{1-\varepsilon}}{\Gamma(\alpha) \Gamma(2-\varepsilon)}\left[\mu_{1}\|x-\zeta\|_{0}+\mu_{2}\|y-\chi\|_{0}\right]+\frac{z_{\alpha} \Omega^{2-\varepsilon}}{\Gamma(\alpha-1) \Gamma(3-\varepsilon)}\left[\mu_{1}\|x-\zeta\|_{0}+\mu_{2}\|y-\chi\|_{0}\right] \\
& +\frac{z_{\alpha} \Omega^{1-\varepsilon}}{\Gamma(\alpha-1) \Gamma(2-\varepsilon)}\left[\mu_{1}\|x-\zeta\|_{0}+\mu_{2}\|y-\chi\|_{0}\right]+\frac{z_{\alpha} \Omega^{1-\varepsilon}}{\Gamma(2-\varepsilon)} \sum_{j=1}^{m} \hat{I}_{1 j}\|x-\zeta\|_{0} \\
& +\frac{z_{\alpha} \Omega^{2-\varepsilon}}{\Gamma(3-\varepsilon)} \sum_{j=1}^{m} \check{I}_{1 j}\|x-\zeta\|_{0}+\frac{z_{\alpha}}{\Gamma(\alpha-1)}\left[\mu_{1}\|x-\zeta\|_{0}+\mu_{2}\|y-\chi\|_{0}\right]+\frac{z_{\alpha}}{\Gamma(\alpha-1)} \\
& \times\left[\mu_{1}\|x-\zeta\|_{0}+\mu_{2}\|y-\chi\|_{0}\right]+\frac{z_{\alpha} \Omega^{1-\varepsilon}}{\Gamma(2-\varepsilon)} \sum_{j=1}^{m} \check{I}_{1 j}\|x-\zeta\|_{0}+z_{\alpha} \sum_{j=1}^{m} \check{I}_{1 j}\|x-\zeta\|_{0} .
\end{aligned}
$$

After some calculation and rearrangement in (38), we get

$$
\|x-\zeta\|_{0}-\frac{\Lambda_{2}}{\left(1-\Lambda_{1}\right)}\|y-x\|_{0} \leqslant \frac{Q_{1} \epsilon_{\alpha}}{\left(1-\Lambda_{1}\right)},
$$

where

$$
\begin{aligned}
\Lambda_{1}= & z_{\alpha}\left[\mu _ { 1 } \left(\frac{1}{z_{\alpha} \Gamma(\alpha+1)}+\frac{1}{\Gamma(\alpha-\varepsilon+1)}+\frac{\Omega^{1-\varepsilon}}{\Gamma(\alpha) \Gamma(2-\varepsilon)}+\frac{\Omega^{2-\varepsilon}}{\Gamma(\alpha-1) \Gamma(3-\varepsilon)}+\frac{\Omega^{1-\varepsilon}}{\Gamma(\alpha-1) \Gamma(2-\varepsilon)}\right.\right. \\
& \left.\left.+\frac{2}{\Gamma(\alpha-1)}\right)+\frac{\Omega^{1-\varepsilon}}{\Gamma(2-\varepsilon)} \sum_{j=1}^{m} \hat{\mathrm{I}}_{1 j}+\frac{\Omega^{2-\varepsilon}}{\Gamma(3-\varepsilon)} \sum_{j=1}^{m} \check{\mathrm{I}}_{1 j}+\frac{\Omega^{1-\varepsilon}}{\Gamma(2-\varepsilon)} \sum_{j=1}^{m} \breve{\mathrm{I}}_{1 j}+\sum_{j=1}^{m} \check{\mathrm{I}}_{1 j}\right], \\
\Lambda_{2}= & z_{\alpha}\left[\mu _ { 2 } \left(\frac{1}{z_{\alpha} \Gamma(\alpha+1)}+\frac{1}{\Gamma(\alpha-\varepsilon+1)}+\frac{\Omega^{1-\varepsilon}}{\Gamma(\alpha) \Gamma(2-\varepsilon)}+\frac{\Omega^{2-\varepsilon}}{\Gamma(\alpha-1) \Gamma(3-\varepsilon)}+\frac{\Omega^{1-\varepsilon}}{\Gamma(\alpha-1) \Gamma(2-\varepsilon)}\right.\right. \\
& \left.\left.+\frac{2}{\Gamma(\alpha-1)}\right)\right] .
\end{aligned}
$$

In addition, for $t \in\left(t_{m}, t_{m+1}\right]$, we have

$$
\begin{aligned}
x(t)= & \frac{1}{\Gamma(\alpha)} \int_{t_{m}}^{t}(t-s)^{\alpha-1}\left[h\left(s,{ }^{c} D^{a} x(s),{ }^{c} D^{b} y(s)\right)+\digamma_{h}(s)\right] d s \\
& +\frac{1}{\Gamma(\alpha)} \sum_{j=1}^{m} \int_{t_{j-1}}^{t_{j}}\left(t_{j}-s\right)^{\alpha-1}\left[h\left(s,{ }^{c} D^{a} x(s),{ }^{c} D^{b} y(s)\right)+\digamma_{h}(s)\right] d s \\
& +\frac{1}{\Gamma(\alpha-1)} \sum_{j=1}^{m}\left(t-t_{j}\right) \int_{t_{j-1}}^{t_{j}}\left(t_{j}-s\right)^{\alpha-2}\left[h\left(s,{ }^{c} D^{a} x(s),{ }^{c} D^{b} y(s)\right)+\digamma_{h}(s)\right] d s \\
& +\frac{1}{2 \Gamma(\alpha-2)} \sum_{j=1}^{m}\left(t-t_{j}\right)^{2} \int_{t_{j-1}}^{t_{j}}\left(t_{j}-s\right)^{\alpha-3}\left[h\left(s,{ }^{c} D^{a} x(s),{ }^{c} D^{b} y(s)\right)+\digamma_{h}(s)\right] d s \\
& +\sum_{j=1}^{m}\left[M_{1 j} x\left(t_{j}\right)+\digamma_{m}\right]+\sum_{j=1}^{m}\left(t-t_{j}\right)\left[N_{1 j}\left(x\left(t_{j}\right)\right)+\digamma_{m}\right] \\
& +\frac{z_{\alpha} t^{2}}{\Gamma(\alpha-\varepsilon)} \int_{t_{j}}^{\Omega}(\Omega-s)^{\alpha-\varepsilon-1}\left[h\left(s,{ }^{c} D^{a} x(s),{ }^{c} D^{b} y(s)\right)+\digamma_{h}(s)\right] d s \\
& +\frac{z_{\alpha} t^{2} \Omega^{1-\varepsilon}}{\Gamma(\alpha-1) \Gamma(2-\varepsilon)} \sum_{j=1}^{m} \int_{t_{j-1}}^{t_{j}}\left(t_{j}-s\right)^{\alpha-2}\left[h\left(s,{ }^{c} D^{a} x(s),{ }^{c} D^{b} y(s)\right)+\digamma_{h}(s)\right] d s
\end{aligned}
$$




$$
\begin{aligned}
& +\frac{z_{\alpha} t^{2} \Omega^{2-\varepsilon}}{\Gamma(\alpha-2) \Gamma(3-\varepsilon)} \sum_{j=1}^{m} \int_{t_{j-1}}^{t_{j}}\left(t_{j}-s\right)^{\alpha-3}\left[h\left(s,{ }^{c} D^{a} x(s),{ }^{c} D^{b} y(s)\right)+\digamma_{h}(s)\right] d s \\
& +\frac{z_{\alpha} t^{2} \Omega^{1-\varepsilon}}{\Gamma(\alpha-2) \Gamma(2-\varepsilon)} \sum_{j=1}^{m} t_{j} \int_{t_{j-1}}^{t_{j}}\left(t_{j}-s\right)^{\alpha-3}\left[h\left(s,{ }^{c} D^{a} x(s),{ }^{c} D^{b} y(s)\right)+\digamma_{h}(s)\right] d s \\
& +\frac{z_{\alpha} t^{2} \Omega^{1-\varepsilon}}{\Gamma(2-\varepsilon)} \sum_{j=1}^{m}\left[N_{1 j}\left(x\left(t_{j}\right)\right)+\digamma_{m}\right]+\frac{z_{\alpha} t^{2} \Omega^{2-\varepsilon}}{\Gamma(3-\varepsilon)} \sum_{j=1}^{m}\left[O_{1 j}\left(x\left(t_{j}\right)\right)+\digamma_{m}\right] \\
& +\frac{z_{\alpha} t^{2}}{\Gamma(\alpha-2)} \int_{t_{m}}^{1}(1-s)^{\alpha-3}\left[h\left(s,{ }_{,}^{c} D^{a} x(s),{ }_{,}^{c} D^{b} y(s)\right)+\digamma_{h}(s)\right] d s \\
& +\frac{z_{\alpha} t^{2}}{\Gamma(\alpha-2)} \sum_{j=1}^{m} \int_{t_{j-1}}^{t_{j}}\left(t_{j}-s\right)^{\alpha-3}\left[h\left(s_{,}{ }^{c} D^{a} x(s),{ }^{c} D^{b} y(s)\right)+\digamma_{h}(s)\right] d s \\
& +\frac{z_{\alpha} t^{2} \Omega^{1-\varepsilon}}{\Gamma(2-\varepsilon)} \sum_{j=1}^{m} t_{j}\left[O_{1 j}\left(x\left(t_{j}\right)\right)+\digamma_{m}\right]+z_{\alpha} t^{2} \sum_{j=1}^{m}\left[O_{1 j}\left(x\left(t_{j}\right)\right)+\digamma_{m}\right] \\
& +\sum_{j=1}^{m} \frac{\left(t-t_{j}\right)^{2}}{2}\left[O_{1 j}\left(x\left(t_{j}\right)\right)+\digamma_{m}\right] .
\end{aligned}
$$

For computational convenience, we use $s_{1}^{*}(t)$ for the sum of terms which are free of $\digamma$, so we have

$$
\begin{aligned}
& \left|x(t)-s_{1}^{*}(t)\right| \\
& \leqslant \frac{1}{\Gamma(\alpha)} \int_{t_{m}}^{t}(t-s)^{\alpha-1}\left|\digamma_{h}(s)\right| d s+\frac{1}{\Gamma(\alpha)} \sum_{j=1}^{m} \int_{t_{j-1}}^{t_{j}}\left(t_{j}-s\right)^{\alpha-1}\left|\digamma_{h}(s)\right| d s \\
& \quad+\frac{1}{\Gamma(\alpha-1)} \sum_{j=1}^{m}\left|\left(t-t_{j}\right)\right| \int_{t_{j-1}}^{t_{j}}\left(t_{j}-s\right)^{\alpha-2}\left|\digamma_{h}(s)\right| d s+\frac{1}{2 \Gamma(\alpha-2)} \sum_{j=1}^{m}\left|\left(t-t_{j}\right)^{2}\right| \int_{t_{j-1}}^{t_{j}}\left(t_{j}-s\right)^{\alpha-3} \\
& \quad \times\left|\digamma_{h}(s)\right| d s+\sum_{j=1}^{m}\left|\digamma_{m}\right|+\sum_{j=1}^{m}\left|\left(t-t_{j}\right)\right|\left|\digamma_{m}\right|+\frac{z_{\alpha}}{\Gamma(\alpha-\varepsilon)} \int_{t_{j}}^{\Omega}(\Omega-s)^{\alpha-\varepsilon-1}\left|\digamma_{h}(s)\right| d s \\
& +\frac{z_{\alpha} \Omega^{1-\varepsilon}}{\Gamma(\alpha-1) \Gamma(2-\varepsilon)} \sum_{j=1}^{m} \int_{t_{j-1}}^{t_{j}}\left(t_{j}-s\right)^{\alpha-2}\left|\digamma_{h}(s)\right| d s+\frac{z_{\alpha} \Omega^{2-\varepsilon}}{\Gamma(\alpha-2) \Gamma(3-\varepsilon)} \sum_{j=1}^{m} \int_{t_{j-1}}^{t_{j}}\left(t_{j}-s\right)^{\alpha-3}\left|\digamma_{h}(s)\right| d s \\
& +\frac{z_{\alpha} \Omega^{1-\varepsilon}}{\Gamma(\alpha-2) \Gamma(2-\varepsilon)} \sum_{j=1}^{m} t_{j} \int_{t_{j-1}}^{t_{j}}\left(t_{j}-s\right)^{\alpha-3}\left|\digamma_{h}(s)\right| d s+\frac{z_{\alpha} \Omega^{1-\varepsilon}}{\Gamma(2-\varepsilon)} \sum_{j=1}^{m}\left|\digamma_{m}\right|+\frac{z_{\alpha} \Omega^{2-\varepsilon}}{\Gamma(3-\varepsilon)} \sum_{j=1}^{m}\left|\digamma_{m}\right| \\
& +\frac{z_{\alpha}}{\Gamma(\alpha-2)} \int_{t_{m}}^{1}(1-s)^{\alpha-3}\left|\digamma_{h}(s)\right| d s+\frac{z_{\alpha}}{\Gamma(\alpha-2)} \sum_{j=1}^{m} \int_{t_{j-1}}^{t_{j}}\left(t_{j}-s\right)^{\alpha-3}\left|\digamma_{h}(s)\right| d s \\
& +\frac{z_{\alpha} \Omega^{1-\varepsilon}}{\Gamma(2-\varepsilon)} \sum_{j=1}^{m}\left|t_{j}\right|\left|\digamma_{m}\right|+z_{\alpha} \sum_{j=1}^{m}\left|\digamma_{m}\right|+\sum_{j=1}^{m} \frac{\left|\left(t-t_{j}\right)^{2}\right|}{2}\left|\digamma_{m}\right| .
\end{aligned}
$$

By utilizing Remark 1, we get

$$
\begin{aligned}
& \left|x(t)-s_{1}^{*}(t)\right| \\
\leqslant & \left(\frac{2}{\Gamma(\alpha+1)}+\frac{1}{\Gamma(\alpha)}+\frac{4 z_{\alpha}+1}{2 \Gamma(\alpha-1)}+\frac{3 z_{\alpha}+4}{2}+\frac{z_{\alpha}}{\Gamma(\alpha-\varepsilon+1)}+\frac{z_{\alpha} \Omega^{1-\varepsilon}}{\Gamma(\alpha) \Gamma(2-\varepsilon)}+\frac{2 z_{\alpha} \Omega^{1-\varepsilon}}{\Gamma(2-\varepsilon)}+\frac{z_{\alpha} \Omega^{2-\varepsilon}}{\Gamma(3-\varepsilon)}\right. \\
& \left.+\frac{z_{\alpha} \Omega^{2-\varepsilon}}{\Gamma(\alpha-1) \Gamma(3-\varepsilon)}+\frac{z_{\alpha} \Omega^{1-\varepsilon}}{\Gamma(\alpha-1) \Gamma(2-\varepsilon)}\right) \epsilon_{\alpha} .
\end{aligned}
$$


For computational convenience, let

$$
\begin{aligned}
Q_{\alpha}= & \frac{2}{\Gamma(\alpha+1)}+\frac{1}{\Gamma(\alpha)}+\frac{4 z_{\alpha}+1}{2 \Gamma(\alpha-1)}+\frac{3 z_{\alpha}+4}{2}+\frac{z_{\alpha}}{\Gamma(\alpha-\varepsilon+1)}+\frac{z_{\alpha} \Omega^{1-\varepsilon}}{\Gamma(\alpha) \Gamma(2-\varepsilon)}+\frac{2 z_{\alpha} \Omega^{1-\varepsilon}}{\Gamma(2-\varepsilon)}+\frac{z_{\alpha} \Omega^{2-\varepsilon}}{\Gamma(3-\varepsilon)} \\
& +\frac{z_{\alpha} \Omega^{2-\varepsilon}}{\Gamma(\alpha-1) \Gamma(3-\varepsilon)}+\frac{z_{\alpha} \Omega^{1-\varepsilon}}{\Gamma(\alpha-1) \Gamma(2-\varepsilon)} .
\end{aligned}
$$

Thus, (40) becomes

$$
\left|x(t)-s_{1}^{*}(t)\right| \leqslant Q_{\alpha} \epsilon_{\alpha}
$$

Let

$$
|x(t)-\zeta(t)|=\left|x(t)-s_{1}^{*}(t)+s_{1}^{*}(t)-\zeta(t)\right| \leqslant\left|x(t)-s_{1}^{*}(t)\right|+\left|s_{1}^{*}(t)-\zeta(t)\right| .
$$

Using (41) in (42), we get

$$
\begin{aligned}
& |x(t)-\zeta(t)| \\
& \leqslant Q_{\alpha} \epsilon_{\alpha}+\frac{1}{\Gamma(\alpha)} \int_{t_{m}}^{t}(t-s)^{\alpha-1}\left|h\left(s,{ }^{c} D^{a} \chi(s),{ }^{c} D^{b} y(s)\right)-h\left(s,{ }^{c} D^{a} \zeta(s),{ }^{c} D^{b} \chi(s)\right)\right| d s \\
& +\frac{1}{\Gamma(\alpha)} \sum_{j=1}^{m} \int_{t_{j-1}}^{t_{j}}\left(t_{j}-s\right)^{\alpha-1}\left|h\left(s,{ }^{c} D^{a} x(s),{ }^{c} D^{b} y(s)\right)-h\left(s,{ }^{c} D^{a} \zeta(s),{ }^{c} D^{b} \chi(s)\right)\right| d s \\
& +\frac{1}{\Gamma(\alpha-1)} \sum_{j=1}^{m}\left|\left(t-t_{j}\right)\right| \int_{t_{j-1}}^{t_{j}}\left(t_{j}-s\right)^{\alpha-2}\left|h\left(s,{ }^{c} D^{a} \chi(s),{ }^{c} D^{b} y(s)\right)-h\left(s,{ }^{c} D^{a} \zeta(s),{ }^{c} D^{b} \chi(s)\right)\right| d s \\
& +\frac{1}{2 \Gamma(\alpha-2)} \sum_{j=1}^{m}\left|\left(t-t_{j}\right)^{2}\right| \int_{t_{j-1}}^{t_{j}}\left(t_{j}-s\right)^{\alpha-3}\left|h\left(s,{ }^{c} D^{a} x(s),{ }_{,}^{c} D^{b} y(s)\right)-h\left(s,{ }^{c} D^{a} \zeta(s),{ }^{c} D^{b} \chi(s)\right)\right| d s \\
& +\sum_{j=1}^{m}\left|M_{1 j}\left(x\left(t_{j}\right)\right)-M_{1 j}\left(\zeta\left(t_{j}\right)\right)\right|+\sum_{j=1}^{m}\left|\left(t-t_{j}\right)\right|\left|N_{1 j}\left(x\left(t_{j}\right)\right)-N_{1 j}\left(\zeta\left(t_{j}\right)\right)\right| \\
& +\frac{z_{\alpha} t^{2}}{\Gamma(\alpha-\varepsilon)} \int_{t_{j}}^{\Omega}(\Omega-s)^{\alpha-\varepsilon-1}\left|h\left(s,{ }^{c} D^{a} x(s),{ }^{c} D^{b} y(s)\right)-h\left(s,{ }^{c} D^{a} \zeta(s),{ }^{c} D^{b} \chi(s)\right)\right| d s \\
& +\frac{z_{\alpha} t^{2} \Omega^{1-\varepsilon}}{\Gamma(\alpha-1) \Gamma(2-\varepsilon)} \sum_{j=1}^{m} \int_{t_{j-1}}^{t_{j}}\left(t_{j}-s\right)^{\alpha-2}\left|h\left(s,{ }^{c} D^{a} \chi(s),{ }^{c} D^{b} y(s)\right)-h\left(s,{ }^{c} D^{a} \zeta(s),{ }^{c} D^{b} \chi(s)\right)\right| d s \\
& +\frac{z_{\alpha} t^{2} \Omega^{2-\varepsilon}}{\Gamma(\alpha-2) \Gamma(3-\varepsilon)} \sum_{j=1}^{m} \int_{t_{j-1}}^{t_{j}}\left(t_{j}-s\right)^{\alpha-3}\left|h\left(s,{ }^{c} D^{a} \chi(s),{ }^{c} D^{b} y(s)\right)-h\left(s,{ }^{c} D^{a} \zeta(s),{ }^{c} D^{b} \chi(s)\right)\right| d s \\
& +\frac{z_{\alpha} t^{2} \Omega^{1-\varepsilon}}{\Gamma(\alpha-2) \Gamma(2-\varepsilon)} \sum_{j=1}^{m}\left|t_{j}\right| \int_{t_{j-1}}^{t_{j}}\left(t_{j}-s\right)^{\alpha-3}\left|h\left(s,{ }^{c} D^{a} x(s),{ }^{c} D^{b} y(s)\right)-h\left(s,{ }^{c} D^{a} \zeta(s),{ }^{c} D^{b} \chi(s)\right)\right| d s \\
& +\frac{z_{\alpha} t^{2} \Omega^{1-\varepsilon}}{\Gamma(2-\varepsilon)} \sum_{j=1}^{m}\left|N_{1 j}\left(x\left(t_{j}\right)\right)-N_{1 j}\left(\zeta\left(t_{j}\right)\right)\right|+\frac{z_{\alpha} t^{2} \Omega^{2-\varepsilon}}{\Gamma(3-\varepsilon)} \sum_{j=1}^{m}\left|O_{1 j}\left(x\left(t_{j}\right)\right)-O_{1 j}\left(\zeta\left(t_{j}\right)\right)\right| \\
& +\frac{z_{\alpha} t^{2}}{\Gamma(\alpha-2)} \int_{t_{m}}^{1}(1-s)^{\alpha-3}\left|h\left(s,{ }^{c} D^{a} x(s),{ }^{c} D^{b} y(s)\right)-h\left(s,{ }^{c} D^{a} \zeta(s),{ }^{c} D^{b} \chi(s)\right)\right| d s \\
& +\frac{z_{\alpha} t^{2}}{\Gamma(\alpha-2)} \sum_{j=1}^{m} \int_{t_{j-1}}^{t_{j}}\left(t_{j}-s\right)^{\alpha-3}\left|h\left(s,{ }^{c} D^{a} x(s),{ }^{c} D^{b} y(s)\right)-h\left(s,{ }^{c} D^{a} \zeta(s),{ }^{c} D^{b} \chi(s)\right)\right| d s
\end{aligned}
$$




$$
\begin{aligned}
& +\frac{z_{\alpha} t^{2} \Omega^{1-\varepsilon}}{\Gamma(2-\varepsilon)} \sum_{j=1}^{m}\left|t_{j}\right|\left|O_{1 j}\left(x\left(t_{j}\right)\right)-O_{1 j}\left(\zeta\left(t_{j}\right)\right)\right|+z_{\alpha} t^{2} \sum_{j=1}^{m}\left|O_{1 j}\left(x\left(t_{j}\right)\right)-O_{1 j}\left(\zeta\left(t_{j}\right)\right)\right| \\
& +\sum_{j=1}^{m} \frac{\left|\left(t-t_{j}\right)^{2}\right|}{2}\left|O_{1 j}\left(x\left(t_{j}\right)\right)-O_{1 j}\left(\zeta\left(t_{j}\right)\right)\right| .
\end{aligned}
$$

Using $\left(\mathbf{M}_{1}\right)$ and $\left(\mathbf{M}_{2}\right)$, we have

$$
\begin{aligned}
& \leqslant Q_{\alpha} \epsilon_{\alpha}+\frac{1}{\Gamma(\alpha+1)}\left[\mu_{1}\|x-\zeta\|_{0}+\mu_{2}\|y-\chi\|_{0}\right]+\frac{1}{\Gamma(\alpha+1)}\left[\mu_{1}\|x-\zeta\|_{0}+\mu_{2}\|y-x\|_{0}\right] \\
& +\frac{1}{\Gamma(\alpha)}\left[\mu_{1}\|x-\zeta\|_{0}+\mu_{2}\|y-\chi\|_{0}\right]+\frac{1}{2 \Gamma(\alpha-1)}\left[\mu_{1}\|x-\zeta\|_{0}+\mu_{2}\|y-x\|_{0}\right] \\
& +\sum_{j=1}^{m} I_{1 j}\|x-\zeta\|_{0}+\sum_{j=1}^{m} \hat{I}_{1 j}\|x-\zeta\|_{0}+\frac{z_{\alpha}}{\Gamma(\alpha-\varepsilon+1)}\left[\mu_{1}\|x-\zeta\|_{0}+\mu_{2}\|y-\chi\|_{0}\right] \\
& +\frac{z_{\alpha} \Omega^{1-\varepsilon}}{\Gamma(\alpha) \Gamma(2-\varepsilon)}\left[\mu_{1}\|x-\zeta\|_{0}+\mu_{2}\|y-\chi\|_{0}\right]+\frac{z_{\alpha} \Omega^{2-\varepsilon}}{\Gamma(\alpha-1) \Gamma(3-\varepsilon)}\left[\mu_{1}\|x-\zeta\|_{0}+\mu_{2}\|y-\chi\|_{0}\right] \\
& +\frac{z_{\alpha} \Omega^{1-\varepsilon}}{\Gamma(\alpha-1) \Gamma(2-\varepsilon)}\left[\mu_{1}\|x-\zeta\|_{0}+\mu_{2}\|y-x\|_{0}\right]+\frac{z_{\alpha} \Omega^{1-\varepsilon}}{\Gamma(2-\varepsilon)} \sum_{j=1}^{m} \hat{I}_{1 j}\|x-\zeta\|_{0} \\
& +\frac{z_{\alpha} \Omega^{2-\varepsilon}}{\Gamma(3-\varepsilon)} \sum_{j=1}^{m} \check{I}_{1 j}\|x-\zeta\|_{0}+\frac{z_{\alpha}}{\Gamma(\alpha-1)}\left[\mu_{1}\|x-\zeta\|_{0}+\mu_{2}\|y-\chi\|_{0}\right] \\
& +\frac{z_{\alpha}}{\Gamma(\alpha-1)}\left[\mu_{1}\|x-\zeta\|_{0}+\mu_{2}\|y-\chi\|_{0}\right]+\frac{z_{\alpha} \Omega^{1-\varepsilon}}{\Gamma(2-\varepsilon)} \sum_{j=1}^{m} \check{I}_{1 j}\|x-\zeta\|_{0}+z_{\alpha} \sum_{j=1}^{m} \check{I}_{1 j}\|x-\zeta\|_{0} \\
& +\frac{1}{2} \sum_{j=1}^{m} \check{I}_{1 j}\|x-\zeta\|_{0} .
\end{aligned}
$$

After some calculation and rearrangement in (43), we get

$$
\|x-\zeta\|_{0}-\frac{\Lambda_{2}^{*}}{\left(1-\Lambda_{1}^{*}\right)}\|y-x\|_{0} \leqslant \frac{Q_{\alpha} \epsilon_{\alpha}}{\left(1-\Lambda_{1}^{*}\right)^{\prime}}
$$

where

$$
\begin{aligned}
\Lambda_{1}^{*}= & {\left[\mu _ { 1 } \left(\frac{2}{\Gamma(\alpha+1)}+\frac{1}{\Gamma(\alpha)}+\frac{1}{2 \Gamma(\alpha-1)}+\frac{z_{\alpha}}{\Gamma(\alpha-\varepsilon+1)}+\frac{z_{\alpha} \Omega^{1-\varepsilon}}{\Gamma(\alpha) \Gamma(2-\varepsilon)}+\frac{z_{\alpha} \Omega^{2-\varepsilon}}{\Gamma(\alpha-1) \Gamma(3-\varepsilon)}\right.\right.} \\
& \left.+\frac{z_{\alpha} \Omega^{1-\varepsilon}}{\Gamma(\alpha-1) \Gamma(2-\varepsilon)}+\frac{2 z_{\alpha}}{\Gamma(\alpha-1)}\right)+\sum_{j=1}^{m} \mathrm{I}_{1 j}+\sum_{j=1}^{m} \hat{\mathrm{I}}_{1 j}+\frac{z_{\alpha} \Omega^{1-\varepsilon}}{\Gamma(2-\varepsilon)} \sum_{j=1}^{m} \hat{\mathrm{I}}_{1 j}+\frac{z_{\alpha} \Omega^{2-\varepsilon}}{\Gamma(3-\varepsilon)} \sum_{j=1}^{m} \check{\mathrm{I}}_{1 j} \\
& \left.+\frac{z_{\alpha} \Omega^{1-\varepsilon}}{\Gamma(2-\varepsilon)} \sum_{j=1}^{m} \breve{\mathrm{I}}_{1 j}+z_{\alpha} \sum_{j=1}^{m} \check{\mathrm{I}}_{1 j}+\frac{1}{2} \sum_{j=1}^{m} \check{\mathrm{I}}_{1 j}\right], \\
\Lambda_{2}^{*}= & \mu_{2}\left(\frac{2}{\Gamma(\alpha+1)}+\frac{1}{\Gamma(\alpha)}+\frac{1}{2 \Gamma(\alpha-1)}+\frac{z_{\alpha}}{\Gamma(\alpha-\varepsilon+1)}+\frac{z_{\alpha} \Omega^{1-\varepsilon}}{\Gamma(\alpha) \Gamma(2-\varepsilon)}+\frac{z_{\alpha} \Omega^{2-\varepsilon}}{\Gamma(\alpha-1) \Gamma(3-\varepsilon)}\right. \\
& \left.+\frac{z_{\alpha} \Omega^{1-\varepsilon}}{\Gamma(\alpha-1) \Gamma(2-\varepsilon)}+\frac{2 z_{\alpha}}{\Gamma(\alpha-1)}\right) .
\end{aligned}
$$

On the similar fashion, for $t \in\left[0, t_{1}\right]$, and utilizing $\left(\mathbf{M}_{1}\right)-\left(\mathbf{M}_{2}\right)$, we can find

$$
\left|y(t)-s_{2}(t)\right| \leqslant 2_{2} \epsilon_{\beta},
$$

where $s_{2}(t)$ are those terms which are free of $\digamma$ and

$$
\begin{aligned}
2_{2} & =\frac{1}{\Gamma(\beta+1)}+\frac{z_{\beta}}{\Gamma(\beta-\rho+1)}+\frac{z_{\beta} \Phi^{1-\rho}}{\Gamma(\beta) \Gamma(2-\rho)}+\frac{z_{\beta} \Phi^{2-\rho}}{\Gamma(\beta-1) \Gamma(3-\rho)}+\frac{z_{\beta} \Phi^{1-\rho}}{\Gamma(\beta-1) \Gamma(2-\rho)}+\frac{2 z_{\beta} \Phi^{1-\rho}}{\Gamma(2-\rho)} \\
& +\frac{z_{\beta} \Phi^{2-\rho}}{\Gamma(3-\rho)}+\frac{2 z_{\beta}}{\Gamma(\beta-1)}+z_{\beta},
\end{aligned}
$$


and

$$
\|y-x\|_{0}-\frac{\Lambda_{4}}{\left(1-\Lambda_{3}\right)}\|x-\zeta\|_{0} \leqslant \frac{Q_{2} \epsilon_{\beta}}{\left(1-\Lambda_{3}\right)}
$$

where

$$
\begin{aligned}
\Lambda_{3}= & z_{\beta}\left[\mu _ { 1 } ^ { \prime } \left(\frac{1}{z_{\beta} \Gamma(\beta+1)}+\frac{1}{\Gamma(\beta-\rho+1)}+\frac{\Phi^{1-\rho}}{\Gamma(\beta) \Gamma(2-\rho)}+\frac{\Phi^{2-\rho}}{\Gamma(\beta-1) \Gamma(3-\rho)}+\frac{\Phi^{1-\rho}}{\Gamma(\beta-1) \Gamma(2-\rho)}\right.\right. \\
& \left.\left.+\frac{2}{\Gamma(\beta-1)}\right)+\frac{\Phi^{1-\rho}}{\Gamma(2-\rho)} \sum_{j=1}^{m} \hat{\mathrm{I}}_{2 j}+\frac{\Phi^{2-\rho}}{\Gamma(3-\rho)} \sum_{j=1}^{m} \check{\mathrm{I}}_{2 j}+\frac{\Phi^{1-\rho}}{\Gamma(2-\rho)} \sum_{j=1}^{m} \check{\mathrm{I}}_{2 j}+\sum_{j=1}^{m} \breve{\mathrm{I}}_{2 j}\right], \\
\Lambda_{4}= & z_{\beta}\left[\mu _ { 2 } ^ { \prime } \left(\frac{1}{z_{\beta} \Gamma(\beta+1)}+\frac{1}{\Gamma(\beta-\rho+1)}+\frac{\Phi^{1-\rho}}{\Gamma(\beta) \Gamma(2-\rho)}+\frac{\Phi^{2-\rho}}{\Gamma(\beta-1) \Gamma(3-\rho)}+\frac{\Phi^{1-\rho}}{\Gamma(\beta-1) \Gamma(2-\rho)}\right.\right. \\
& \left.\left.+\frac{2}{\Gamma(\beta-1)}\right)\right] .
\end{aligned}
$$

In addition, for $t \in\left(t_{m}, t_{m+1}\right], 1 \leqslant m \leqslant n$, we can get

$$
\left|y(t)-s_{2}^{*}(t)\right| \leqslant Q_{\beta} \epsilon_{\beta},
$$

where $s_{2}^{*}(t)$ are those terms which are free of $\digamma$ and

$$
\begin{aligned}
Q_{\beta}= & \frac{2}{\Gamma(\beta+1)}+\frac{1}{\Gamma(\beta)}+\frac{4 z_{\beta}+1}{2 \Gamma(\beta-1)}+\frac{3 z_{\beta}+4}{2}+\frac{z_{\beta}}{\Gamma(\beta-\rho+1)}+\frac{z_{\beta} \Phi^{1-\rho}}{\Gamma(\beta) \Gamma(2-\rho)}+\frac{2 z_{\beta} \Phi^{1-\rho}}{\Gamma(2-\rho)}+\frac{z_{\beta} \Phi^{2-\rho}}{\Gamma(3-\rho)} \\
& +\frac{z_{\beta} \Phi^{2-\rho}}{\Gamma(\beta-1) \Gamma(3-\rho)}+\frac{z_{\beta} \Phi^{1-\rho}}{\Gamma(\beta-1) \Gamma(2-\rho)} .
\end{aligned}
$$

In addition,

$$
\|y-x\|_{0}-\frac{\Lambda_{4}^{*}}{\left(1-\Lambda_{3}^{*}\right)}\|x-\zeta\|_{0} \leqslant \frac{Q_{\beta} \epsilon_{\beta}}{\left(1-\Lambda_{3}^{*}\right)^{\prime}}
$$

where

$$
\begin{aligned}
\Lambda_{3}^{*}= & {\left[\mu _ { 2 } ^ { \prime } \left(\frac{2}{\Gamma(\beta+1)}+\frac{1}{\Gamma(\beta)}+\frac{1}{2 \Gamma(\beta-1)}+\frac{z_{\beta}}{\Gamma(\beta-\rho+1)}+\frac{z_{\beta} \Phi^{1-\rho}}{\Gamma(\beta) \Gamma(2-\rho)}+\frac{z_{\beta} \Phi^{2-\rho}}{\Gamma(\beta-1) \Gamma(3-\rho)}\right.\right.} \\
& \left.+\frac{z_{\beta} \Phi^{1-\rho}}{\Gamma(\beta-1) \Gamma(2-\rho)}+\frac{2 z_{\beta}}{\Gamma(\beta-1)}\right)+\sum_{j=1}^{m} \mathrm{I}_{2 j}+\sum_{j=1}^{m} \hat{\mathrm{I}}_{2 j}+\frac{z_{\beta} \Phi^{1-\rho}}{\Gamma(2-\rho)} \sum_{j=1}^{m} \hat{\mathrm{I}}_{2 j}+\frac{z_{\beta} \Phi^{2-\rho}}{\Gamma(3-\rho)} \sum_{j=1}^{m} \breve{\mathrm{I}}_{2 j} \\
& \left.+\frac{z_{\beta} \Phi^{1-\rho}}{\Gamma(2-\rho)} \sum_{j=1}^{m} \breve{\mathrm{I}}_{2 j}+z_{\beta} \sum_{j=1}^{m} \breve{\mathrm{I}}_{2 j}+\frac{1}{2} \sum_{j=1}^{m} \breve{\mathrm{I}}_{2 j}\right], \\
\Lambda_{4}^{*}= & \mu_{1}^{\prime}\left(\frac{2}{\Gamma(\beta+1)}+\frac{1}{\Gamma(\beta)}+\frac{1}{2 \Gamma(\beta-1)}+\frac{z_{\beta}}{\Gamma(\beta-\rho+1)}+\frac{z_{\beta} \Phi^{1-\rho}}{\Gamma(\beta) \Gamma(2-\rho)}+\frac{z_{\beta} \Phi^{2-\rho}}{\Gamma(\beta-1) \Gamma(3-\rho)}\right. \\
& \left.+\frac{z_{\beta} \Phi^{1-\rho}}{\Gamma(\beta-1) \Gamma(2-\rho)}+\frac{2 z_{\beta}}{\Gamma(\beta-1)}\right) .
\end{aligned}
$$

The equivalent matrix of Equations (44) and (48) is given as:

$$
\left[\begin{array}{cc}
1 & -\frac{\Lambda_{2}^{*}}{\left(1-\Lambda_{1}^{*}\right)} \\
-\frac{\Lambda_{4}^{*}}{\left(1-\Lambda_{3}^{*}\right)} & 1
\end{array}\right]\left[\begin{array}{l}
\|x-\zeta\|_{0} \\
\|y-x\|_{0}
\end{array}\right] \leqslant\left[\begin{array}{c}
\frac{Q_{\alpha} \epsilon_{\alpha}}{\left(1-\Lambda_{1}^{*}\right)} \\
\frac{Q_{\beta} \epsilon_{\beta}}{\left(1-\Lambda_{3}^{*}\right)}
\end{array}\right] .
$$


Solving the above inequality, we get

$$
\left[\begin{array}{c}
\|x-\zeta\|_{0} \\
\|y-x\|_{0}
\end{array}\right] \leqslant\left[\begin{array}{cc}
\frac{1}{\Lambda_{0}} & \frac{\Lambda_{2}^{*}}{\Lambda_{0}\left(1-\Lambda_{1}^{*}\right)} \\
\frac{\Lambda_{4}^{*}}{\Lambda_{0}\left(1-\Lambda_{3}^{*}\right)} & \frac{1}{\Lambda_{0}}
\end{array}\right]\left[\begin{array}{c}
\frac{Q_{\alpha} \epsilon_{\alpha}}{\left(1-\Lambda_{1}^{*}\right)} \\
\frac{Q_{\beta} \epsilon_{\beta}}{\left(1-\Lambda_{3}^{*}\right)}
\end{array}\right],
$$

where

$$
\Lambda_{0}=1-\frac{\Lambda_{2}^{*} \Lambda_{4}^{*}}{\left(1-\Lambda_{1}^{*}\right)\left(1-\Lambda_{3}^{*}\right)}>0
$$

Further simplification of the above system gives

$$
\begin{aligned}
\|x-\zeta\|_{0} & \leqslant \frac{Q_{\alpha} \epsilon_{\alpha}}{\Lambda_{0}\left(1-\Lambda_{1}^{*}\right)}+\frac{\Lambda_{2}^{*} Q_{\beta} \epsilon_{\beta}}{\Lambda_{0}\left(1-\Lambda_{1}^{*}\right)\left(1-\Lambda_{3}^{*}\right)}, \\
\|y-\chi\|_{0} & \leqslant \frac{Q_{\beta} \epsilon_{\beta}}{\Lambda_{0}\left(1-\Lambda_{3}^{*}\right)}+\frac{\Lambda_{4}^{*} Q_{\alpha} \epsilon_{\alpha}}{\Lambda_{0}\left(1-\Lambda_{1}^{*}\right)\left(1-\Lambda_{3}^{*}\right)},
\end{aligned}
$$

from which we have

$$
\|x-\zeta\|_{0}+\|y-x\|_{0} \leqslant \frac{Q_{\alpha} \epsilon_{\alpha}}{\Lambda_{0}\left(1-\Lambda_{1}^{*}\right)}+\frac{Q_{\beta} \epsilon_{\beta}}{\Lambda_{0}\left(1-\Lambda_{3}^{*}\right)}+\frac{\Lambda_{4}^{*} Q_{\alpha} \epsilon_{\alpha}}{\Lambda_{0}\left(1-\Lambda_{1}^{*}\right)\left(1-\Lambda_{3}^{*}\right)}+\frac{\Lambda_{2}^{*} Q_{\beta} \epsilon_{\beta}}{\Lambda_{0}\left(1-\Lambda_{1}^{*}\right)\left(1-\Lambda_{3}^{*}\right)} .
$$

Let $\max \left\{\epsilon_{\alpha}, \epsilon_{\beta}\right\}=\epsilon$; then, from (49), we get

$$
\|(x-\zeta, y-x)\| \leqslant \mathbf{K}_{\alpha, \beta} \epsilon,
$$

which implies that

$$
\|(x, y)-(\zeta, x)\| \leqslant \mathbf{K}_{\alpha, \beta} \epsilon
$$

where

$$
\mathbf{K}_{\alpha, \beta}=\left[\frac{Q_{\alpha}}{\Lambda_{0}\left(1-\Lambda_{1}^{*}\right)}+\frac{Q_{\beta}}{\Lambda_{0}\left(1-\Lambda_{3}^{*}\right)}+\frac{\Lambda_{4}^{*} Q_{\alpha}}{\Lambda_{0}\left(1-\Lambda_{1}^{*}\right)\left(1-\Lambda_{3}^{*}\right)}+\frac{\Lambda_{2}^{*} Q_{\beta}}{\Lambda_{0}\left(1-\Lambda_{1}^{*}\right)\left(1-\Lambda_{3}^{*}\right)}\right]
$$

Hence, problem (1) is Ulam-Hyers stable. Moreover, if we set $\Theta(\epsilon)=\mathbf{K}_{x, y} \epsilon ; \Theta(0)=0$ in (50), then problem (1) is generalized Ulam-Hyers stable.

$\left(\mathbf{M}_{5}\right)$ : Let $\Psi_{\alpha}, \Psi_{\beta} \in \mathrm{PC}\left(\mathrm{J}, \mathbb{R}^{+}\right)$be nondecreasing functions; then, for $\mathrm{t} \in \mathrm{J}$, there are $\lambda_{\alpha}, \lambda_{\beta}>0$ such that

$$
\begin{aligned}
I^{\alpha} \Psi_{\alpha}(t) \leqslant \lambda_{\alpha} \Psi_{\alpha}(t), & I^{\alpha-1} \Psi_{\alpha}(t) \leqslant \lambda_{\alpha} \Psi_{\alpha}(t), \\
I^{\alpha-2} \Psi_{\alpha}(t) \leqslant \lambda_{\alpha} \Psi_{\alpha}(t), & I^{\alpha-\varepsilon} \Psi_{\alpha}(t) \leqslant \lambda_{\alpha} \Psi_{\alpha}(t) .
\end{aligned}
$$

Similarly,

$$
\begin{aligned}
I^{\beta} \Psi_{\beta}(t) \leqslant \lambda_{\beta} \Psi_{\beta}(t), & I^{\beta-1} \Psi_{\beta}(t) \leqslant \lambda_{\beta} \Psi_{\beta}(t), \\
I^{\beta-2} \Psi_{\beta}(t) \leqslant \lambda_{\beta} \Psi_{\beta}(t), & I^{\beta-\rho} \Psi_{\beta}(t) \leqslant \lambda_{\beta} \Psi_{\beta}(t) .
\end{aligned}
$$

Theorem 5. Assume that $\left(\mathbf{M}_{1}\right)-\left(\mathbf{M}_{2}\right)$ and $\left(\mathbf{M}_{5}\right)$ are satisfied; then, by Definition 6 and Definition 7 , Problem (1) is Ulam-Hyers-Rassias stable with respect to $\left(\Psi_{\alpha, \beta}, \varphi_{\alpha, \beta}\right)$, as well as generalized Ulam-HyersRassias stable. 


\section{Example}

To substantiate the aforemention demonstrated theory, we supply the following problem:

$$
\left\{\begin{array}{l}
{ }^{c} D^{\alpha} x(t)+h\left(t^{c}{ }^{c} D^{a} x(t),{ }^{c} D^{b} y(t)\right)=0, t \neq t_{m} \\
{ }^{c} D^{\beta} y(t)+w\left(t,{ }^{c} D^{a} x(t),{ }^{c} D^{b} y(t)\right)=0, \\
\left.\Delta x\right|_{t=t_{m}}=M_{1 m} x\left(t_{m}\right),\left.\Delta x^{\prime}\right|_{t=t_{m}}=N_{1 m} x\left(t_{m}\right),\left.\Delta x^{\prime \prime}\right|_{t=t_{m}}=O_{1 m} x\left(t_{m}\right), \\
\left.\Delta y\right|_{t=t_{m}}=M_{2 m} y\left(t_{m}\right),\left.\Delta y^{\prime}\right|_{t=t_{m}}=N_{2 m} y\left(t_{m}\right),\left.\Delta y^{\prime \prime}\right|_{t=t_{m}}=O_{2 m} y\left(t_{m}\right), \\
x(t)={ }^{\prime} x(t)=0,{ }^{c} D^{\varepsilon} x(\Omega)=x^{\prime \prime}(1), y(0)=y^{\prime}(0)=0,{ }^{c} D^{\rho} y(\Phi)=y^{\prime \prime}(1), \\
0<\varepsilon, \Omega<1,0<\rho, \Phi<1 .
\end{array}\right.
$$

Take $J=[0,1], \alpha=\frac{5}{2}, \beta=\sqrt{5}, \varepsilon=\frac{1}{2}, \Omega=\frac{1}{3}, \mathrm{a}=\frac{1}{2}, \mathrm{~b}=\frac{1}{8}, \mathrm{t}_{1}=\frac{1}{2}, \rho=\frac{1}{3}, \Phi=\frac{1}{5}, \mathrm{~h}(\mathrm{t}, \mathrm{x}, \mathrm{y})=$ $e^{t}+\frac{|x|+|y|}{40(1+|x|+|y|)}, w(t, x, y)=\frac{\cos t+|x|+|y|}{40(1+|x|+|y|)}, M_{11}(x)=M_{21}(x)=\frac{|x|}{5+|x|}, N_{11}(x)=N_{21}(x)=\frac{|x|}{10+|x|}$, $\mathrm{O}_{11}(\mathrm{x})=\mathrm{O}_{21}(\mathrm{x})=\frac{|\mathrm{x}|}{15+|\mathrm{x}|}$. By direct computation, we have $\mu_{1}=\mu_{2}=\mu_{1}^{\prime}=\mu_{2}^{\prime}=\frac{1}{40}, \mathrm{I}_{11}=\mathrm{I}_{21}=\frac{1}{5}$, $\hat{\mathrm{I}}_{11}=\hat{\mathrm{I}}_{21}=\frac{1}{10}, \breve{\mathrm{I}}_{11}=\check{\mathrm{I}}_{21}=\frac{1}{15}$,

$$
\begin{aligned}
& {\left[( \mu _ { 1 } + \mu _ { 2 } ) \left(\frac{2}{\Gamma(\alpha+1)}+\frac{1}{\Gamma(\alpha)}+\frac{1}{2 \Gamma(\alpha-1)}+\frac{z_{\alpha}}{\Gamma(\alpha-\varepsilon+1)}+\frac{z_{\alpha} \Omega^{1-\varepsilon}}{\Gamma(\alpha) \Gamma(2-\varepsilon)}+\frac{z_{\alpha} \Omega^{2-\varepsilon}}{\Gamma(\alpha-1) \Gamma(3-\varepsilon)}\right.\right.} \\
& \left.+\frac{z_{\alpha} \Omega^{1-\varepsilon}}{\Gamma(\alpha-1) \Gamma(2-\varepsilon)}+\frac{2 z_{\alpha}}{\Gamma(\alpha-1)}\right)+\sum_{j=1}^{m} \mathrm{I}_{1 j}+\sum_{j=1}^{m} \hat{\mathrm{I}}_{1 j}+\frac{z_{\alpha} \Omega^{1-\varepsilon}}{\Gamma(2-\varepsilon)} \sum_{j=1}^{m} \hat{\mathrm{I}}_{1 j}+\frac{z_{\alpha} \Omega^{2-\varepsilon}}{\Gamma(3-\varepsilon)} \sum_{j=1}^{m} \check{\mathrm{I}}_{1 j} \\
& \left.+\frac{z_{\alpha} \Omega^{1-\varepsilon}}{\Gamma(2-\varepsilon)} \sum_{j=1}^{m} \check{\mathrm{I}}_{1 j}+z_{\alpha} \sum_{j=1}^{m} \check{\mathrm{I}}_{1 j}+\frac{1}{2} \sum_{j=1}^{m} \check{\mathrm{I}}_{1 j}\right] \approx 0.680277<1 .
\end{aligned}
$$

Similarly,

$$
\begin{aligned}
& {\left[( \mu _ { 1 } ^ { \prime } + \mu _ { 2 } ^ { \prime } ) \left(\frac{2}{\Gamma(\beta+1)}+\frac{1}{\Gamma(\beta)}+\frac{1}{2 \Gamma(\beta-1)}+\frac{z_{\beta}}{\Gamma(\beta-\rho+1)}+\frac{z_{\beta} \Phi^{1-\rho}}{\Gamma(\beta) \Gamma(2-\rho)}+\frac{z_{\beta} \Phi^{2-\rho}}{\Gamma(\beta-1) \Gamma(3-\rho)}\right.\right.} \\
& \left.+\frac{z_{\beta} \Phi^{1-\rho}}{\Gamma(\beta-1) \Gamma(2-\rho)}+\frac{2 z_{\beta}}{\Gamma(\beta-1)}\right)+\sum_{j=1}^{m} \mathrm{I}_{2 j}+\sum_{j=1}^{m} \hat{\mathrm{I}}_{2 j}+\frac{z_{\beta} \Phi^{1-\rho}}{\Gamma(2-\rho)} \sum_{j=1}^{m} \hat{\mathrm{I}}_{2 j}+\frac{z_{\beta} \Phi^{2-\rho}}{\Gamma(3-\rho)} \sum_{j=1}^{m} \breve{\mathrm{I}}_{2 j} \\
& \left.+\frac{z_{\beta} \Phi^{1-\rho}}{\Gamma(2-\rho)} \sum_{j=1}^{m} \breve{\mathrm{I}}_{2 j}+z_{\beta} \sum_{j=1}^{m} \breve{\mathrm{I}}_{2 j}+\frac{1}{2} \sum_{j=1}^{m} \breve{I}_{2 j}\right] \approx 0.427420<1 .
\end{aligned}
$$

Thus, by Theorem 2, Problem (51) has a unique solution. Furthermore, all of the assumptions are satisfied, so the Problem (51) is Ulam-Hyers, generalized Ulam-Hyers, Ulam-Hyers-Rassias and generalized Ulam-Hyers-Rassias stable.

\section{Conclusions}

In the above study, we have successfully built up existence theory for the solutions of system (1). The required analysis has been developed with the help of the Banach contraction principle and Schauder fixed point theorem. We found that the fractional order coupled system is additionally complicated and challenging as compared to the single FDEs. We also concluded that, if we increase the order or boundary conditions, then the end result turns into extra accurate. Our results are new and fascinating. Our methods can be used to study the existence of solutions for the high order or multiple-point boundary value systems of a nonlinear coupled system of FDEs. Furthermore, we have presented different kinds of Ulam-Hyers stability results for the solution of the considered system (1). In addition, we have presented our main theoretical results with the help of an example. In the 
future, this concept can be extended to more applied and complicated problems of applied nature. The obtained results can be used in fields like numerical analysis and managerial sciences including business mathematics and economics, etc.

Author Contributions: S.F. and Z.A. contributed equally in writing this article; supervision, A.Z., J.X. and Y.C. All authors read and approved the final manuscript.

Funding: This work is supported by the Talent Project of Chongqing Normal University (Grant No. 02030307-0040), the National Natural Science Foundation of China (Grant No. 11601048, 11571207), the Natural Science Foundation of Chongqing Normal University (Grant No. 16XYY24), the Shandong Natural Science Foundation (ZR2018MA011), and the Tai'shan Scholar Engineering Construction Fund of Shandong Province of China.

Conflicts of Interest: The authors declare that they have no competing interests.

\section{References}

1. Brikaa, M. Existence results for a couple system of nonlinear fractional differential equation with three point boundary conditions. J. Fract. Calc. Appl. 2015, 3, 1-10.

2. Henderson, J.; Luca, R. Positive solutions for a system of fractional differential equations with coupled integral boundary conditions. Appl. Math. Comput. 2014, 249, 182-197. [CrossRef]

3. Kilbas, A.; Srivastava, H.; Trujillo, J. Theory and Applications of Fractional Differential Equations. In North-Holland Mathematics Studies; Elsevier: Amsterdam, The Netherlands, 2006; Volume 204.

4. Klafter, J.; Lim, S.C. Fractional Dynamics in Physics; Metzler, R., Ed.; World Scientific: Singapore, 2011.

5. Podlubny, I. Fractional Differential Equations; Academic Press: San Diego, CA, USA, 1999.

6. Yang, W. Positive solutions for a coupled system of nonlinear fractional differential equations with integral boundary conditions. Comput. Math. Appl. 2012, 63, 288-297. [CrossRef]

7. Zhang, K.; Fu, Z. Solutions for a class of Hadamard fractional boundary value problems with sign-changing nonlinearity. J. Funct. Spaces 2019, 2019, 9046472. [CrossRef]

8. Zhang, K.; Wang, J.; Ma, W. Solutions for integral boundary value problems of nonlinear Hadamard fractional differential equations. J. Funct. Spaces 2018, 2018, 2193234. [CrossRef]

9. Zou, Y.; He, G. On the uniqueness of solutions for a class of fractional differential equations. Appl. Math. Lett. 2017, 74, 68-73. [CrossRef]

10. Yue, Z.; Zou, Y. New uniqueness results for fractional differential equation with dependence on the first order derivative. Adv. Differ. Equ. 2019, 2019, 38. [CrossRef]

11. Fu, Z.; Bai, S.; O'Regan, D.; Xu, J. Nontrivial solutions for an integral boundary value problem involving Riemann-Liouville fractional derivatives. J. Inequal. Appl. 2019, 2019, 104. [CrossRef]

12. Zhang, K.; O'Regan, D.; Xu, J.; Fu, Z. Nontrivial solutions for a higher order nonlinear fractional boundary value problem involving Riemann-Liouville fractional derivatives. J. Funct. Spaces 2019, 2019, 2381530. [CrossRef]

13. Pu, R.; Zhang, X.; Cui, Y.; Li, P.; Wang, W. Positive solutions for singular semipositone fractional differential equation subject to multipoint boundary conditions. J. Funct. Spaces 2017, 2017, 5892616. [CrossRef]

14. Zhang, Y. Existence results for a coupled system of nonlinear fractional multi-point boundary value problems at resonance. J. Inequal. Appl. 2018, 2018, 198. [CrossRef] [PubMed]

15. Zhang, Y.; Bai, Z.; Feng, T. Existence results for a coupled system of nonlinear fractional three-point boundary value problems at resonance. Comput. Math. Appl. 2011, 61, 1032-1047. [CrossRef]

16. Qi, T.; Liu, Y.; Zou, Y. Existence result for a class of coupled fractional differential systems with integral boundary value conditions. J. Nonlinear Sci. Appl. 2017, 10, 4034-4045. [CrossRef]

17. Qi, T.; Liu, Y.; Cui, Y. Existence of solutions for a class of coupled fractional differential systems with nonlocal boundary conditions. J. Funct. Spaces 2017, 2017, 6703860. [CrossRef]

18. Xu, J.; Goodrich, C.S.; Cui, Y. Positive solutions for a system of first-order discrete fractional boundary value problems with semipositone nonlinearities. Revista de la Real Academia de Ciencias Exactas Físicas y Naturales Serie A Matemáticas 2019, 113, 1343-1358. [CrossRef]

19. Cheng, W.; Xu, J.; Cui, Y.; Ge, Q. Positive solutions for a class of fractional difference systems with coupled boundary conditions. Adv. Differ. Equ. 2019, 2019, 249. [CrossRef]

20. Cheng, W.; Xu, J.; Cui, Y. Positive solutions for a system of nonlinear semipositone fractional q-difference equations with q-integral boundary conditions. J. Nonlinear Sci. Appl. 2017, 10, 4430-4440. [CrossRef] 
21. Wang, F.; Cui, Y. Positive solutions for an infinite system of fractional order boundary value problems. Adv. Differ. Equ. 2019, 2019, 169. [CrossRef]

22. Qiu, X.; Xu, J.; O’Regan, D.; Cui, Y. Positive solutions for a system of nonlinear semipositone boundary value problems with Riemann-Liouville fractional derivatives. J. Funct. Spaces 2018, 2018, 7351653. [CrossRef]

23. Hao, X.; Wang, H.; Liu, L.; Cui, Y. Positive solutions for a system of nonlinear fractional nonlocal boundary value problems with parameters and p-Laplacian operator. Bound. Value Probl. 2017, 2017, 182. [CrossRef]

24. Zhang, X.; Liu, L.; Zou, Y. Fixed-point theorems for systems of operator equations and their applications to the fractional differential equations. J. Funct. Spaces 2018, 2018, 7469868. [CrossRef]

25. Zhang, X.; Liu, L.; Wu, Y.; Zou, Y. Existence and uniqueness of solutions for systems of fractional differential equations with Riemann-Stieltjes integral boundary condition. Adv. Differ. Equ. 2018, 2018, 204. [CrossRef]

26. Li, H.; Zhang, J. Positive solutions for a system of fractional differential equations with two parameters. J. Funct. Spaces 2018, 2018, 1462505. [CrossRef]

27. Zhao, Y.; Hou, X.; Sun, Y.; Bai, Z. Solvability for some class of multi-order nonlinear fractional systems. Adv. Differ. Equ. 2019, 2019, 23. [CrossRef]

28. Zhao, Y.; Sun, Y.; Wang, Y.; Bai, Z. Asymptotical stabilization of the nonlinear upper triangular fractionalorder systems. Adv. Differ. Equ. 2019, 2019, 157. [CrossRef]

29. Cui, Y. Multiplicity results for positive solutions to differential systems of singular coupled integral boundary value problems. Math. Probl. Eng. 2017, 2017, 3608352. [CrossRef]

30. Jiang, J.; O’Regan, D.; Xu, J.; Fu, Z. Positive solutions for a system of nonlinear Hadamard fractional differential equations involving coupled integral boundary conditions. J. Inequal. Appl. 2019, 2019, 204. [CrossRef]

31. Zhai, C.; Wang, W.; Li, H. A uniqueness method to a new Hadamard fractional differential system with four-point boundary conditions. J. Inequal. Appl. 2018, 2018, 207. [CrossRef]

32. Wang, J.; Fečkan, M.; Zhou, Y. A survey on impulsive fractional differential equations. Fract. Calc. Appl. Anal. 2016, 19, 806-831. [CrossRef]

33. Yukunthorn, W.; Ahmad, B.; Ntouyas, S.; Tariboon, J. On Caputo-Hadamard type fractional impulsive hybrid systems with nonlinear fractional integral conditions. Nonlinear Anal. Hybrid Syst. 2016, 19, 77-92. [CrossRef]

34. Zhao, K.; Liang, J. Solvability of triple-point integral boundary value problems for a class of impulsive fractional differential equations. Adv. Differ. Equ. 2017, 2017, 50. [CrossRef]

35. $\mathrm{Fu}, \mathrm{X}$; Bao, X. Some existence results for nonlinear fractional differential equations with impulsive and fractional integral boundary conditions. Adv. Differ. Equ. 2014, 2014, 129. [CrossRef]

36. Zhao, K. Multiple positive solutions of integral BVPs for high-order nonlinear fractional differential equations with impulses and distributed delays. Dyn. Syst. 2015, 30, 208-223. [CrossRef]

37. Zhao, K. Impulsive boundary value problems for two classes of fractional differential equation with two different Caputo fractional derivatives. Mediterr. J. Math. 2016, 13, 1033-1050. [CrossRef]

38. Wang, Y.; Liu, Y.; Cui, Y. Infinitely many solutions for impulsive fractional boundary value problem with p-Laplacian. Bound. Value Probl. 2018, 2018, 94. [CrossRef]

39. Zuo, M.; Hao, X.; Liu, L.; Cui, Y. Existence results for impulsive fractional integro-differential equation of mixed type with constant coefficient and antiperiodic boundary conditions. Bound. Value Probl. 2017, 2017, 161. [CrossRef]

40. Bai, Z.; Dong, X.; Yin, C. Existence results for impulsive nonlinear fractional differential equation with mixed boundary conditions. Bound. Value Probl. 2016, 2016, 63. [CrossRef]

41. Ulam, S.M. Problems in Modern Mathematics; John Wiley and Sons: New York, NY, USA, 1940.

42. Ulam, S.M. A Collection of Mathematical Problems; Interscience: New York, NY, USA, 1960.

43. Rassias, T.M. On the stability of the linear mapping in Banach spaces. Proc. Am. Math. Soc. 1978, 72, $297-300$. [CrossRef]

44. Ali, Z.; Zada, A.; Shah, K. Ulam stability to a toppled systems of nonlinear implicit fractional order boundary value problem. Bound. Value Probl. 2018, 2018, 175. [CrossRef]

45. Ali, Z.; Zada, A.; Shah, K. On Ulam's stability for a coupled systems of nonlinear implicit fractional differential equations. Bull. Malays. Math. Sci. Soc. 2019, 42, 2681-2699. [CrossRef]

46. Ahmad, N.; Ali, Z.; Shah, K.; Zada, A.; Rahman, G. Analysis of implicit type nonlinear dynamical problem of impulsive fractional differential equations. Complexity 2018, 2018, 6423974. [CrossRef] 
47. Zada, A.; Shah, S.O.; Shah, R. Hyers-Ulam stability of non-autonomous systems in terms of boundedness of Cauchy problem. Appl. Math. Comput. 2015, 271, 512-518. [CrossRef]

48. Shah, S.O.; Zada, A. Existence, uniqueness and stability of solution to mixed integral dynamic systems with instantaneous and noninstantaneous impulses on time scales. Appl. Math. Comput. 2019, 359, 202-213. [CrossRef]

49. Zada, A.; Mashal, A. Stability analysis of $\mathrm{n}^{\text {th }}$ order nonlinear impulsive differential equations in Quasi-Banach space. Numer. Funct. Anal. Optim. 2019. [CrossRef]

50. Ali, A.; Rabiei, F.; Shah, K. On Ulam's type stability for a class of impulsive fractional differential equations with nonlinear integral boundary conditions. J. Nonlinear Sci. Appl. 2017, 10, 4760-4775. [CrossRef]

51. Riaz, U.; Zada, A.; Ali, Z.; Ahmad, M.; Xu, J.; Fu, Z. Analysis of nonlinear coupled systems of impulsive fractional differential equations with Hadamard derivatives. Math. Probl. Eng. 2019, 2019, 5093572. [CrossRef]

52. Riaz, U.; Zada, A.; Ali, Z.; Cui, Y.; Xu, J. Analysis of coupled systems of implicit impulsive fractional differential equations involving Hadamard derivatives. Adv. Differ. Equ. 2019, 2019, 226. [CrossRef]

53. Ali, Z.; Kumam, P.; Shah, K.; Zada, A. Investigation of Ulam stability results of a coupled system of nonlinear implicit fractional differential equations. Mathematics 2019, 7, 341. [CrossRef]

54. Wang, J.; Zada, A.; Waheed, H. Stability analysis of a coupled system of nonlinear implicit fractional anti-periodic boundary value problem. Math. Meth. App. Sci. 2019. [CrossRef]

55. Shah, K.; Shah, L.; Ahmad, S.; Rassias, J.M.; Li, Y. Monotone iterative techniques together with Hyers-UlamRassias stability. Math. Meth. Appl. Sci. 2019,1-18. [CrossRef]

56. Zada, A.; Ali, W.; Park, C. Ulam's type stability of higher order nonlinear delay differential equations via integral inequality of Grönwall-Bellman-Bihari's type. Appl. Math. Comput. 2019, 350, 60-65.

57. Jung, S.M. Hyers-Ulam-Rassias Stability of Functional Equations in Nonlinear Analysis; Springer: New York, NY, USA, 2011.

58. Kumam, P.; Ali, A.; Shah, K.; Khan, R.A. Existence results and Hyers-Ulam stability to a class of nonlinear arbitrary order differential equations. J. Nonlinear Sci. Appl. 2017, 10, 2986-2997. [CrossRef]

59. Guo, D.; Lakshmikantham, V. Nonlinear Problems in Abstract Cone; Academic Press: Orlando, FL, USA, 1988.

60. Miller, K.; Ross, B. An Introduction to the Fractional Calculus and Fractional Differential Equations; Wiley: New York, NY, USA, 1993.

61. Rus, I.A. Ulam stabilities of ordinary differential equations in a Banachspace. Carpathian J. Math. 2010, 26, 103-107.

(C) 2019 by the authors. Licensee MDPI, Basel, Switzerland. This article is an open access article distributed under the terms and conditions of the Creative Commons Attribution (CC BY) license (http:/ / creativecommons.org/licenses/by/4.0/). 